\title{
HydroSource Data Dictionary
}

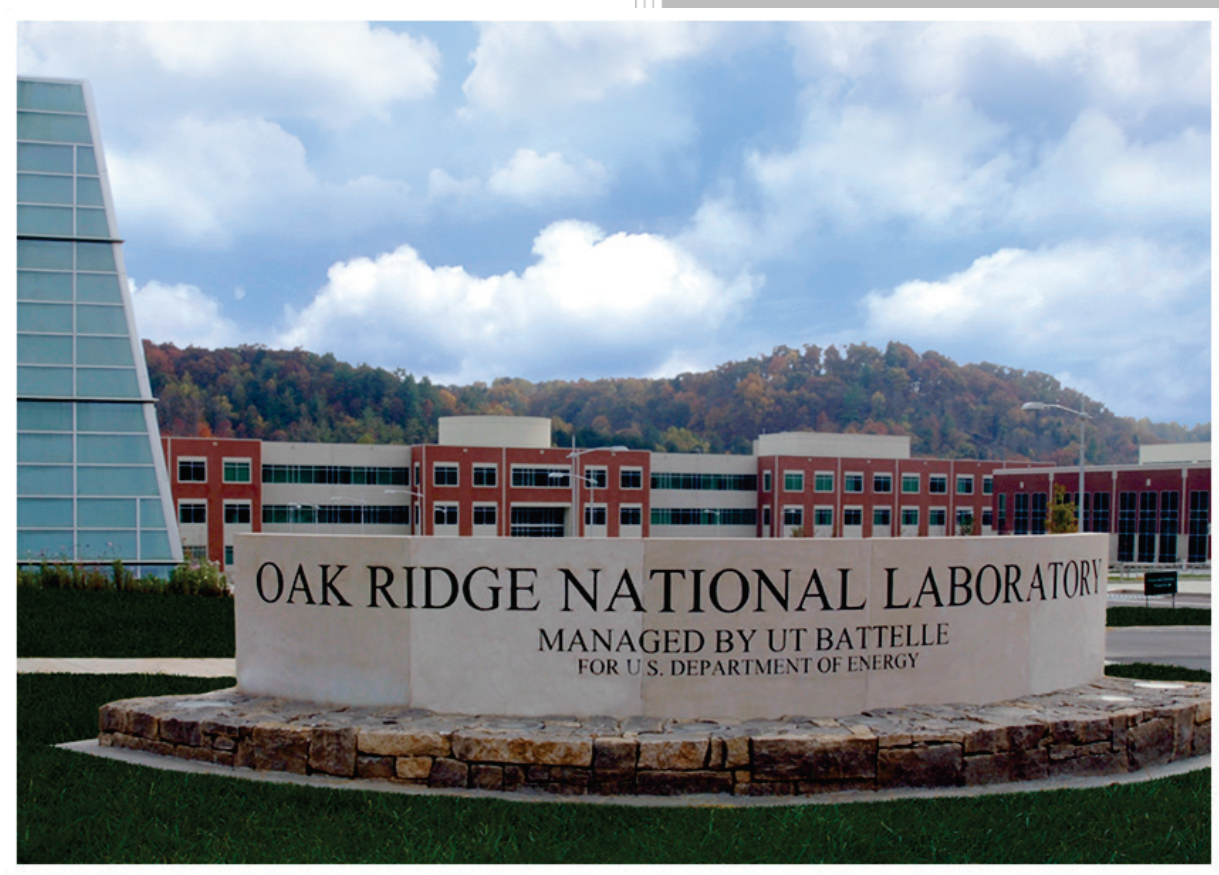

Approved for public release.

Brennan T. Smith

Nicole Samu

Shelaine Curd

Yaxing Wei

Zhaoying Wei

Distribution is unlimited.

March 2019 


\title{
DOCUMENT AVAILABILITY
}

Reports produced after January 1, 1996, are generally available free via US Department of Energy (DOE) SciTech Connect.

Website www.osti.gov

Reports produced before January 1, 1996, may be purchased by members of the public from the following source:

\author{
National Technical Information Service \\ 5285 Port Royal Road \\ Springfield, VA 22161 \\ Telephone 703-605-6000 (1-800-553-6847) \\ TDD 703-487-4639 \\ Fax 703-605-6900 \\ E-mail info@ntis.gov \\ Website http://classic.ntis.gov/
}

Reports are available to DOE employees, DOE contractors, Energy Technology Data Exchange representatives, and International Nuclear Information System representatives from the following source:

Office of Scientific and Technical Information

PO Box 62

Oak Ridge, TN 37831

Telephone 865-576-8401

Fax 865-576-5728

E-mail reports@osti.gov

Website http://www.osti.gov/contact.html

\begin{abstract}
This report was prepared as an account of work sponsored by an agency of the United States Government. Neither the United States Government nor any agency thereof, nor any of their employees, makes any warranty, express or implied, or assumes any legal liability or responsibility for the accuracy, completeness, or usefulness of any information, apparatus, product, or process disclosed, or represents that its use would not infringe privately owned rights. Reference herein to any specific commercial product, process, or service by trade name, trademark, manufacturer, or otherwise, does not necessarily constitute or imply its endorsement, recommendation, or favoring by the United States Government or any agency thereof. The views and opinions of authors expressed herein do not necessarily state or reflect those of the United States Government or any agency thereof.
\end{abstract}


Environmental Sciences Division

\title{
HydroSource Data Dictionary
}

\author{
Brenann T. Smith \\ Nicole Samu \\ Shelaine Curd \\ Yaxing Wei \\ Zhaoying Wei
}

Date Published: March 2019

Prepared by

OAK RIDGE NATIONAL LABORATORY

Oak Ridge, TN 37831-6283

managed by

UT-BATTELLE, LLC

for the

US DEPARTMENT OF ENERGY

under contract DE-AC05-00OR22725 



\section{CONTENTS}

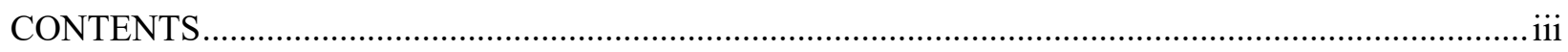

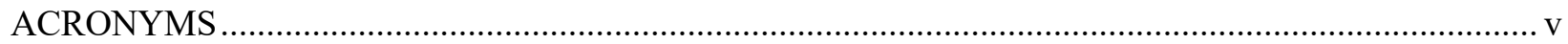

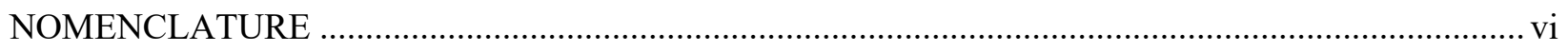

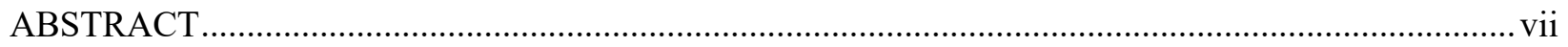

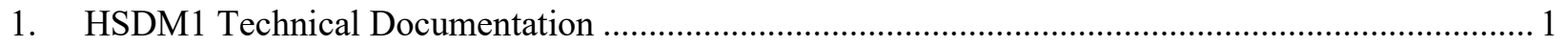

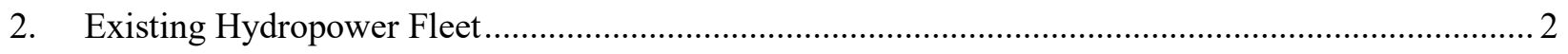

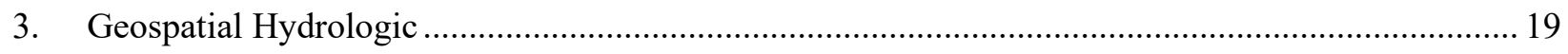

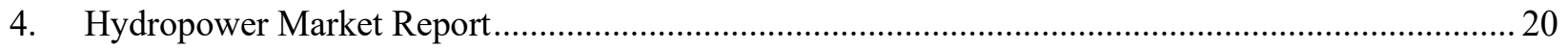

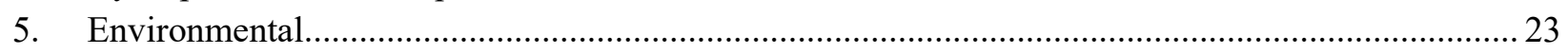

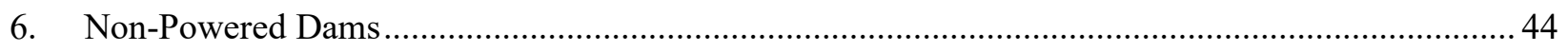

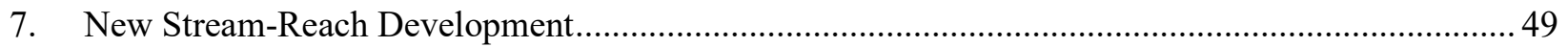

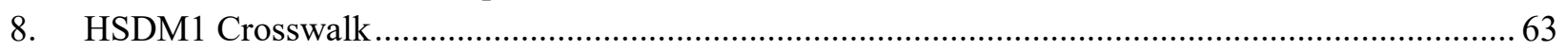

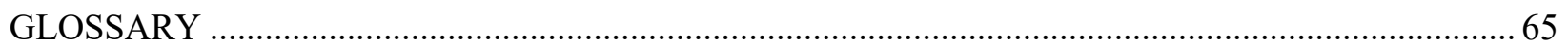




\section{ACRONYMS}

\begin{tabular}{|c|c|}
\hline $\mathrm{CI}$ & condition index \\
\hline DAMS & FERC Dam Safety and Inspection's Database \\
\hline DO & Dissolved Oxygen \\
\hline DOE & US Department of Energy \\
\hline EF & existing fleet \\
\hline EIA & Energy Information Administration \\
\hline EPA & US Environmental Protection Agency \\
\hline ESA & Endangered Species Act \\
\hline ESC & eastern stream classification \\
\hline EUCG & Electric Utility Cost Group \\
\hline FEMA & Federal Emergency Management Agency \\
\hline FERC & Federal Energy Regulatory Commission \\
\hline GADS & Generating Availability Data System \\
\hline HMR & Hydropower Market Report \\
\hline HS & HydroSource component of the HydroSource Data Model \\
\hline HSDM1 & HydroSource Data Model, Version 1 \\
\hline HUC & hydrologic unit code \\
\hline IIR & Industrial Information Resources (database) \\
\hline IUCN & International Union for the Conservation of Nature \\
\hline MRLC & Multi-Resolution Land Characteristics Consortium \\
\hline NERC & North American Electric Reliability Corporation \\
\hline NHD & National Hydrography Dataset \\
\hline NHDPlus & National Hydrography Dataset Plus \\
\hline NID & National Inventory of Dams \\
\hline NLCD & National Land Cover Dataset \\
\hline NPD & non-powered dam \\
\hline NSD & new stream-reach development \\
\hline NWIS & National Water Information System \\
\hline ORNL & Oak Ridge National Laboratory \\
\hline PCA & power control area \\
\hline PS & Pumped Storage \\
\hline $\mathrm{R} \& \mathrm{U}$ & Refurbishments and Upgrades \\
\hline SMH & Standard Modular Hydropower (Explorer) \\
\hline USGS & US Geological Survey \\
\hline WPTO & Water Power Technologies Office \\
\hline
\end{tabular}




\section{NOMENCLATURE}

\begin{tabular}{clll}
\hline Abbreviation & Measure & Alternate name & Description \\
\hline $\mathrm{ac}-\mathrm{ft}$ & Volume & & acre-feet \\
$\mathrm{cfs}$ & Flow & Volumetric flux & cubic feet per second \\
$\mathrm{cm}$ & Length & Length & centimeters \\
$\mathrm{dd}$ & Angle of Arc & & decimal degrees \\
$\mathrm{fps}$ & Speed & Velocity magnitude & feet per second \\
$\mathrm{ft}$ & Length & & feet \\
$\mathrm{m}$ & Length & & meters \\
$\mathrm{MW}$ & Power & Generating capacity & megawatts \\
$\mathrm{MWh}$ & Energy & Energy production & megawatt-hours \\
$\%$ & Ratio & Percent & percent
\end{tabular}




\begin{abstract}
In recent years, Oak Ridge National Laboratory (ORNL) has conducted innovative hydropower research sponsored by the US Department of Energy Water Power Technologies Office (WPTO) to address important questions regarding technology advancement, existing hydropower assets, future hydropower potential, environmental concerns, policy and regulatory decisions, and market trends to support hydropower sustainability in the United States. Data from this research supports a broad and diverse user base including US federal, state, and local governments; national laboratories; hydropower industry; academia; nonprofit organizations; and other end users. The development of a relational database system is needed to ensure proper management of the continuously increasing breadth and depth of these data to guarantee their future value and reliability for advancing hydropower research and supporting the goals and objectives of our partners and US hydropower stakeholders.

During FY 2018, ORNL's HydroSource team reconciled data from ORNL's WPTO-funded projects to create the HydroSource Data Model, Version 1 (HSDM1) schema. The HSDM1 schema provides the essential step in data assimilation toward unifying ORNL's WPTO-funded data into a relational database management system. This report provides technical documentation of the HSDM1 schema to help guide future development and implementation of the HydroSource database system.
\end{abstract}

\title{
HydroSource Data Model, Version 1 - Database Schema
}

A Relational Database System for US Water Power

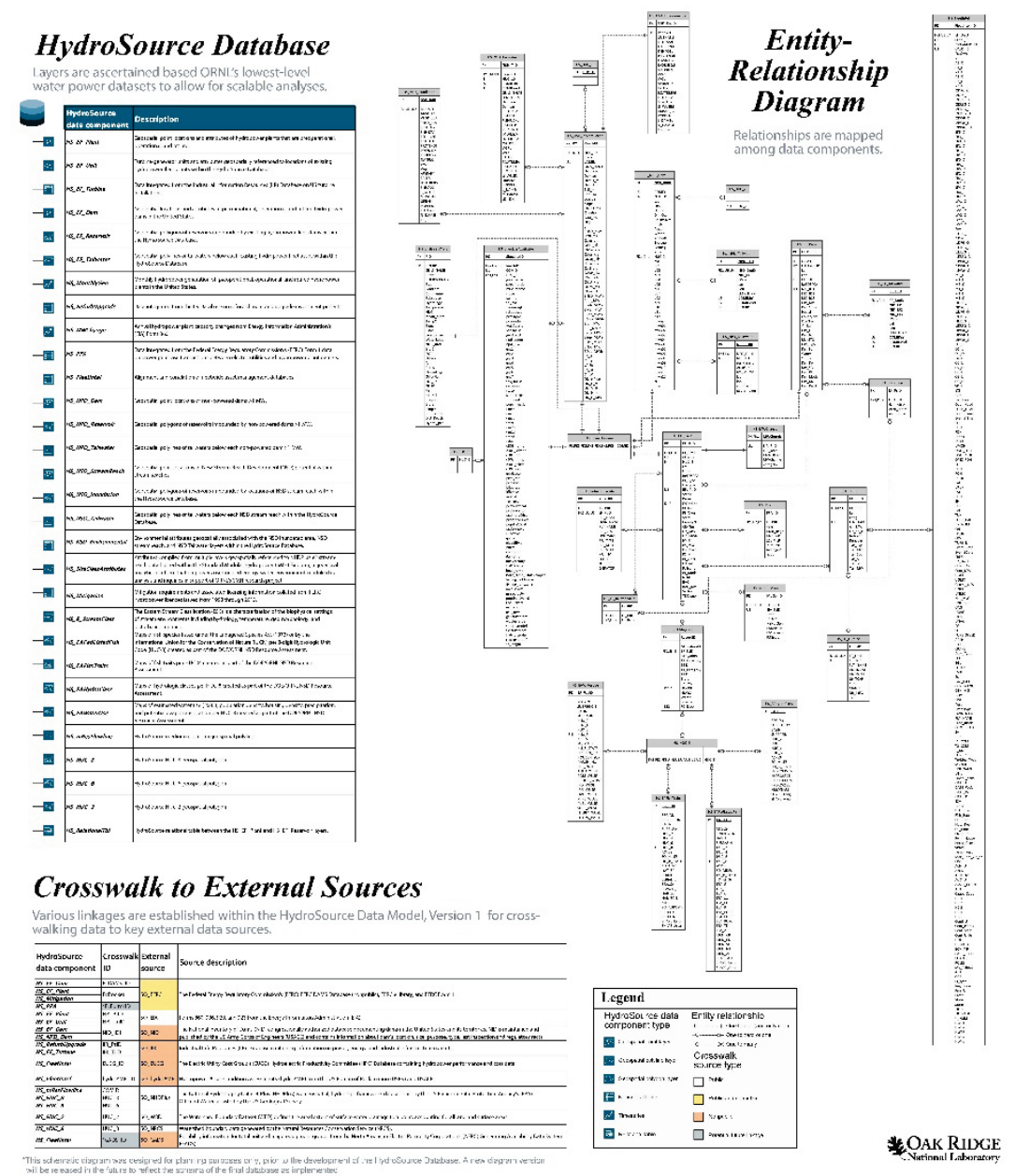




\section{SDM1 TECHNICAL DOCUMENTATION}

Here we introduce the data structure and associations that compose the HydroSource Data Model, Version 1 (HSDM1) schema. Our primary intent is to develop a prototype model schema based on existing data resulting from US Department of Energy (DOE) Water Power Technologies Office (WPTO)-funded projects at Oak Ridge National Laboratory (ORNL). This schema provides the necessary step in data assimilation for organizing WPTO-funded ORNL data into a logical framework upon which the HydroSource database system can be effectively planned and developed.

Data components are the primary building blocks that make up the HSDM1 schema. They are essentially different types of data objects that store (1) related sets of attributes, and (2) database indices (i.e., primary key, foreign key, external crosswalk key, and potential future key) that provide connectivity among and beyond data components within HSDM1. We categorize different types of data components within HSDM1 based on their general forms and functions, respectively (i.e., geospatial point layer, geospatial polyline layer, geospatial polygon layer, nonspatial table, time-series, and relational table), to characterize how they explicitly model different types of information.

In the following tables, we group HSDM1 documentation under section headings based on different research themes and logical data component groupings. Under each heading, we first summarize top-level information about data components to document and identify their corresponding field names, aliases, types, descriptions, and confidentiality. This information allows us to quickly and easily identify and differentiate among major sets of data (i.e., data components) within HSDM1. Next, we document properties (i.e., field, alias, unit of measurement, description) of the database indices and sets of attributes associated with each data component. Last but not least, we introduce the HSDMI Crosswalk, which provides capabilities to extend the HSDM1 through key linkages to major external data frameworks that are relevant for supporting US hydropower sustainability. Ultimately, we hope that this documentation will effectively align multiple perspectives to help facilitate the continued development and deployment of the HydroSource database system. 


\section{EXISTING HYDROPOWER FLEET}

HSDM: HS_DG: Summary of existing fleet (EF) data components

\begin{tabular}{|c|c|c|c|}
\hline Data_Component & Alias & Type & Description \\
\hline HS_EF_Plant & EF plant & $\begin{array}{l}\text { Geospatial point } \\
\text { layer }\end{array}$ & $\begin{array}{l}\text { Geospatial point locations and attributes of } \\
\text { hydropower plants that are preoperational, } \\
\text { operational, and retired }\end{array}$ \\
\hline HS_EF_Unit & EF unit & $\begin{array}{l}\text { Geospatial point } \\
\text { layer }\end{array}$ & $\begin{array}{l}\text { Turbine-generator units and attributes geospatially } \\
\text { referenced to locations of existing hydropower fleet } \\
\text { plants within the HydroSource Database }\end{array}$ \\
\hline HS_EF_Turbine & EF turbine & Nonspatial table & $\begin{array}{l}\text { Data integrated from the Industrial Information } \\
\text { Resources (IIR) Database on US turbine installations }\end{array}$ \\
\hline HS_EF_Dam & EF dam & $\begin{array}{l}\text { Geospatial point } \\
\text { layer }\end{array}$ & $\begin{array}{l}\text { Geospatial locations and attributes of preoperational, } \\
\text { operational, and retired hydropower dams in the } \\
\text { United States }\end{array}$ \\
\hline HS_EF_Reservoir & EF reservoir & $\begin{array}{l}\text { Geospatial } \\
\text { polygon layer }\end{array}$ & $\begin{array}{l}\text { Geospatial polygons of reservoirs impounded by } \\
\text { existing hydropower fleet dams within the } \\
\text { HydroSource Database }\end{array}$ \\
\hline HS_EF_Tailwater & EF tailwater & $\begin{array}{l}\text { Geospatial } \\
\text { polyline layer }\end{array}$ & $\begin{array}{l}\text { Geospatial polylines of tailwaters below each existing } \\
\text { hydropower fleet asset within the HydroSource } \\
\text { Database }\end{array}$ \\
\hline HS_FleetIntel & $\begin{array}{l}\text { Hydro fleet } \\
\text { intelligence }\end{array}$ & Nonspatial table & $\begin{array}{l}\text { Alignment and correlation of fleetwide asset } \\
\text { management databases }\end{array}$ \\
\hline
\end{tabular}


HS_EF_Plant: Attribute summary

\begin{tabular}{|c|c|c|c|}
\hline Field Name & Alias & Unit & Description \\
\hline$\overline{\text { EF_PtID }}$ & Existing Fleet Plant ID & ID & $\begin{array}{l}\text { Unique identification code assigned to each HS_EF_Plant } \\
\text { record }\end{array}$ \\
\hline EF_DmID & Existing Fleet Dam ID & ID & $\begin{array}{l}\text { Unique identification code assigned to each HS_EF_Dam } \\
\text { record }\end{array}$ \\
\hline COMID & $\begin{array}{l}\text { NHDPlus V1 Common } \\
\text { Identifier }\end{array}$ & ID & $\begin{array}{l}\text { National Hydrography Dataset Plus (NHDPlus) V1 } \\
\text { flowline common identifier }\end{array}$ \\
\hline Lat & Latitude & $\mathrm{DD}$ & Latitude \\
\hline Long & Longitude & $\mathrm{DD}$ & Longitude \\
\hline HUC_8 & 8-Digit HUC & ID & $\begin{array}{l}\text { Eight-digit US Geological Survey (USGS) hydrologic unit } \\
\text { code (HUC) }\end{array}$ \\
\hline ReEDSPCA & ReEDS PCA & N/A & $\begin{array}{l}\text { Power control area (PCA) assigned to a plant based on the } \\
\text { location of the existing powerhouse }\end{array}$ \\
\hline Pt_Type & Plant Type & N/A & Type of hydropower facility \\
\hline OwType & Plant Ownership Category & N/A & Hydropower plant ownership category \\
\hline EIA_PtID & EIA Plant ID & ID & Energy Information Administration (EIA) Plant ID \\
\hline Status & Project Status & N/A & Current status of the hydropower project \\
\hline Permit & Permit Type & N/A & Type of hydropower permit \\
\hline FcDocket & FERC Docket Number & ID & $\begin{array}{l}\text { Federal Energy Regulatory Commission (FERC) docket } \\
\text { number }\end{array}$ \\
\hline Pt_Nm & Plant Name & N/A & Plant name \\
\hline Pt_Own & Plant Owner & N/A & Plant owner \\
\hline State & USPS 2-letter postal code & N/A & $\begin{array}{l}\text { Two-letter US Postal Service abbreviations of US states } \\
\text { or associated territories that each powerplant is within }\end{array}$ \\
\hline CountyNm & County name & $\mathrm{N} / \mathrm{A}$ & County the powerplant is within \\
\hline CityNm & City name & $\mathrm{N} / \mathrm{A}$ & Neighboring city \\
\hline HY_MW & $\begin{array}{l}\text { Hydropower nameplate } \\
\text { capacity }\end{array}$ & MW & $\begin{array}{l}\text { Total capacity from hydraulic turbine-generator units } \\
\text { within each plant. Excludes capacity from pumped storage } \\
\text { (PS) turbine-generator units. PS turbine-generator units } \\
\text { are those which can consume (instead of generating) } \\
\text { power to pump water to an upper reservoir for later use }\end{array}$ \\
\hline HY_MWh & Hydropower net generation & MWh & Average annual net hydropower generation \\
\hline
\end{tabular}




\begin{tabular}{|c|c|c|c|}
\hline HY_Pf & Hydropower plant factor & $\%$ & $\begin{array}{l}\text { Hydropower plant factor for each plant calculated as (total } \\
\text { hydropower net generation-plant consumption)/ } \\
\text { (hydropower capacity } \times \text { hours in a year) }\end{array}$ \\
\hline PS_MW & $\begin{array}{l}\text { Pumped storage nameplate } \\
\text { capacity }\end{array}$ & MW & $\begin{array}{l}\text { Pumped storage nameplate capacity in megawatts for each } \\
\text { powerplant }\end{array}$ \\
\hline PS_MWh & $\begin{array}{l}\text { Pumped storage gross } \\
\text { generation }\end{array}$ & MWh & $\begin{array}{l}\text { Pumped storage gross generation in megawatt hours for } \\
\text { each powerplant }\end{array}$ \\
\hline PS_Pf & Pumped storage plant factor & $\%$ & Pumped storage plant factor for each plant \\
\hline Pt_OnYr & Plant year in service & YYYY & Year that the first generator came online \\
\hline FcIssue & FERC permit issue date & $\mathrm{N} / \mathrm{A}$ & FERC date that either a license or exemption was issued \\
\hline FcExpire & FERC permit expiration date & $\mathrm{N} / \mathrm{A}$ & FERC license expiration date \\
\hline Pt_Mode & Mode of operation & $\mathrm{N} / \mathrm{A}$ & Mode of operation classification of the plant \\
\hline NERC & NERC region & $\mathrm{N} / \mathrm{A}$ & $\begin{array}{l}\text { North American Electric Reliability Corporation } \\
\text { (NERC) region each plant falls within }\end{array}$ \\
\hline $\mathrm{BAC}$ & Balancing authority code & $\mathrm{N} / \mathrm{A}$ & $\begin{array}{l}\text { Identifier code of the balancing authority for each } \\
\text { hydropower plant }\end{array}$ \\
\hline BAC_Nm & Balancing authority name & $\mathrm{N} / \mathrm{A}$ & $\begin{array}{l}\text { Name of the balancing authority for each hydropower } \\
\text { plant }\end{array}$ \\
\hline Sector & Sector name & $\mathrm{N} / \mathrm{A}$ & Plant-level sector name defined by the EIA \\
\hline TDOwner & $\begin{array}{l}\text { Transmission or Distribution } \\
\text { System Owner }\end{array}$ & $\mathrm{N} / \mathrm{A}$ & $\begin{array}{l}\text { Transmission or distribution system owner of the energy } \\
\text { generated by the hydropower plant }\end{array}$ \\
\hline
\end{tabular}


HS_EF_Unit: Attribute summary

\begin{tabular}{llll}
\hline \multicolumn{1}{c}{ Field Name } & \multicolumn{1}{c}{ Alias } & \multicolumn{1}{c}{ Unit } & \multicolumn{1}{c}{ Description } \\
\hline EF_UnID & Existing fleet unit id & ID & $\begin{array}{l}\text { Unique identification code assigned to each HS_EF_Unit } \\
\text { record }\end{array}$ \\
EF_PtID & Existing fleet plant id & ID & $\begin{array}{l}\text { Unique identification code assigned to each HS_EF_Plant } \\
\text { record }\end{array}$ \\
Lat & Latitude & DD & Latitude \\
Long & Longitude & DD & Longitude \\
EIA_UnID & EIA unit id & ID & EIA turbine-generator unit ID \\
Gn_MW & Nameplate capacity & MW & Nameplate capacity for each generator \\
Gn_Yr_IS & Generator Year in Service & YYYY & Year generator came online \\
RetireYr & Year retired & YYYY & Year generator retired \\
RehabYr & Year rehabilitated & YYYY & Year turbine rehabilitated \\
Tur_Typ & Turbine type & N/A & Type of turbine \\
Tur_Man & Turbine manufacturer & N/A & Turbine manufacturer \\
Tur_Spd & Turbine speed & rmp & Turbine speed \\
Tur_h & Turbine rated head & ft & Change in water levels between intake and discharge point \\
Tur_hp & Turbine power & hp & Turbine power \\
Tur_MW & Turbine hydraulic capacity & cfs & Maximum turbine hydraulic capacity \\
\hline
\end{tabular}


HS_EF_Turbine: Attribute summary

\begin{tabular}{llll}
\hline \multicolumn{1}{c}{ Alias } & \multicolumn{1}{c}{ Field Name } & Unit & \multicolumn{1}{c}{ Description } \\
\hline Existing Fleet Unit ID & EF_TrID & ID & Unique identification code assigned to each HS_EF_Turbine record \\
Existing Fleet Plant ID & EF_UnID & ID & Unique identification code assigned to each HS_EF_Unit record \\
IIR Plant ID & IIR_TrID & ID & $\begin{array}{l}\text { Unique identification code assigned to each turbine-level record } \\
\text { within the IIR Database }\end{array}$ \\
Plant name & PLT_NAME & N/A & Name of plant \\
Unit name & UNITNAME & N/A & Name of unit \\
Unit state & UNITSTATE & N/A & Unit location (state) \\
Unit capacity & UNITCAP & MW & Capacity of installed unit \\
Start year & YEAR & N/A & Year in which the new turbine starts operation \\
Unit manufacturer & MANUF & N/A & Turbine manufacturer \\
Turbine drive details & DR_INFO & N/A & Turbine drive details \\
Turbine type & TYPE & N/A & Turbine drive type \\
\hline
\end{tabular}

\section{HS_EF_Dam: Attribute summary}

\begin{tabular}{llll}
\hline \multicolumn{1}{c}{ Alias } & \multicolumn{1}{c}{ Field Name } & Unit & \multicolumn{1}{c}{ Description } \\
\hline Existing Fleet Dam ID & EF_DmID & ID & Unique identification code assigned to each HS_EF_Dam record \\
FERC DAMS Dam ID & FcDAMS_ID & ID & $\begin{array}{l}\text { Unique identifier associated with dams from the FERC Dam } \\
\text { Safety and Inspections Database (DAMS) }\end{array}$ \\
$\begin{array}{llll}\text { NHDPlus V1 Common } \\
\text { Identifier }\end{array}$ & COMID & ID & NHDPlus V1 flowline common identifier \\
Latitude & Lat & DD & Latitude \\
Longitude & Long & DD & Longitude \\
8-Digit HUC & HUC_8 & ID & $\begin{array}{l}\text { Eight-digit USGS HUC } \\
\text { ReEDS PCA }\end{array}$ \\
ReEDSPCA & ID & $\begin{array}{l}\text { PCA assigned to a plant based on the location of the existing } \\
\text { powerhouse }\end{array}$ \\
NID_ID1 & NID_ID1 & ID & $\begin{array}{l}\text { Unique identification code assigned to each dam record within } \\
\text { the National Inventory of Dams (NID) Database }\end{array}$
\end{tabular}




\begin{tabular}{|c|c|c|c|}
\hline NID State ID & NID_ID2 & ID & $\begin{array}{l}\text { State or federal agency identification code for each dam record } \\
\text { within the NID Database }\end{array}$ \\
\hline NID Structure ID & NID_ID3 & ID & $\begin{array}{l}\text { Identification code for saddle dams or dike associated with the } \\
\text { larger dam project }\end{array}$ \\
\hline Dam Name & Dm_Nm & N/A & Name of the dam \\
\hline Dam Owner Name & Dm_Own & N/A & Owner of the dam \\
\hline $\begin{array}{l}\text { USPS 2-Letter Postal } \\
\text { Code }\end{array}$ & Postal & N/A & $\begin{array}{l}\text { Two-letter US Postal Service abbreviations of US states or } \\
\text { associated territories each powerplant is within }\end{array}$ \\
\hline County Name & CountyNm & N/A & County dam is within \\
\hline City Name & CityNm & N/A & City dam is within \\
\hline Year Built & Yr_Built & YYYY & Year the dam was built \\
\hline Dam Height & Dm_HT & $\mathrm{ft}$ & Height of the dam \\
\hline Dam Length & Dm_LG & $\mathrm{ft}$ & Length of the dam \\
\hline Maximum Storage & MaxSTO & ac-ft & Maximum storage of the dam \\
\hline Dam Type & Dm_Type & N/A & Type of dam \\
\hline Dam Purpose & Dm_Pur & N/A & Purpose for the dam \\
\hline Watersource & Water & N/A & Primary water source of the dam \\
\hline Annual Mean Flow & Yr_cfs & $\mathrm{cfs}$ & Estimated annual mean flow \\
\hline Drainage Area & Dm_DA & $\mathrm{mi}^{\wedge} 2$ & Drainage area of the dam \\
\hline Surface Area & Dm_SA & $\mathrm{ac}$ & Surface area of the impoundment at its normal retention level \\
\hline Residence Time & Dm_RT & day & $\begin{array}{l}\text { Residence time, estimated by } \mathrm{d} \times(\text { Reservoir storage in ac } \times \mathrm{ft}) / \\
\text { (annual mean flow in } \mathrm{cfs} \text { ) } \mathrm{d}=0.504 \text { is a unit conversion factor }\end{array}$ \\
\hline Mode of Operation & Dm_Mode & N/A & Mode of operation classification for the dam \\
\hline Diversion & Dm_Div & N/A & Denotes whether or not the dam contains a diversion \\
\hline Diversion Length & Dm_DivLG & $\mathrm{mi}$ & Length of the diversion \\
\hline
\end{tabular}


HS_EF_Reservoir: Attribute summary

\begin{tabular}{|c|c|c|c|}
\hline Alias & Field Name & Unit & Description \\
\hline $\begin{array}{l}\text { Existing Fleet } \\
\text { Reservoir ID }\end{array}$ & EF_RsID & ID & Unique identification code assigned to each HS_EF_Reservoir record. \\
\hline $\begin{array}{l}\text { Existing Fleet Dam } \\
\text { ID }\end{array}$ & EF_DmID & ID & Unique identification code assigned to each HS_EF_Dam record. \\
\hline $\begin{array}{l}\text { Dam Distance } \\
\text { Kilometers }\end{array}$ & dam_distkm & $\mathrm{km}$ & $\begin{array}{l}\text { Nearest distance from dam to edge of reservoir. If intersecting reservoir, } \\
\text { then value is } 0 .\end{array}$ \\
\hline $\begin{array}{l}\text { Area Square } \\
\text { Kilometers }\end{array}$ & Area_SQKM & $\mathrm{km}^{2}$ & Reservoir area in square kilometers. \\
\hline Reservoir Source & res_source & NA & $\begin{array}{l}\text { Source of information used to create the reservoir (either NHDPlus } \\
\text { V1"Waterbody," NHDPlus V1 "Area," or digitized and converted into } \\
\text { polygons from NLCD open water land cover classification. }\end{array}$ \\
\hline
\end{tabular}

HS_EF_Tailwater: Attribute summary

\begin{tabular}{|c|c|c|c|}
\hline Alias & Field Name & Unit & Description \\
\hline $\begin{array}{l}\text { Existing Fleet } \\
\text { Tailwater ID }\end{array}$ & EF_TIID & ID & $\begin{array}{l}\text { Unique identification code assigned to each } \\
\text { HS_EF_Tailwater record }\end{array}$ \\
\hline $\begin{array}{l}\text { Existing Fleet Dam } \\
\text { ID }\end{array}$ & EF_DmID & ID & $\begin{array}{l}\text { Unique identification code assigned to each } \mathrm{HS}_{-} \mathrm{EF}_{-} \text {Unit } \\
\text { record }\end{array}$ \\
\hline NID ID & NID_ID1 & ID & Official NID ID for the dam \\
\hline NID State ID & NID_ID2 & ID & NID state or federal agency ID for the dam \\
\hline NID Structure ID & NID_ID3 & ID & $\begin{array}{l}\text { NID ID for the saddle dam or dike associated with the } \\
\text { larger dam project }\end{array}$ \\
\hline Dam Name & Water & N/A & Name of dam \\
\hline Latitude & Lat & Decimal degrees & Latitude of dam \\
\hline Longitude & Lon & Decimal degrees & Longitude of dam \\
\hline Length $\mathrm{Km}$ & LENGTHKM & Kilometers & Total length of each tailwater in kilometers \\
\hline COMID Initial & COMIDini & NA & $\begin{array}{l}\text { Unique identification code from NHDPlus V1 for each } \\
\text { stream reach at the upstream end of the tailwater }\end{array}$ \\
\hline COMID End & COMIDend & NA & $\begin{array}{l}\text { Unique identification code from NHDPlus V1 for each } \\
\text { stream reach at the downstream end of the tailwater }\end{array}$ \\
\hline COMID & COMID & NA & $\begin{array}{l}\text { Unique identification code from NHDPlus V1 for each } \\
\text { stream reach at the location of the dam }\end{array}$ \\
\hline
\end{tabular}


HS_FleetIntel: Attribute summary

\begin{tabular}{|c|c|c|c|}
\hline Alias & Field Name & Unit & Description \\
\hline Hydro Fleet Intelligence ID & FleetIntel_ID & ID & $\begin{array}{l}\text { Unique identification code assigned to } \\
\text { each HS_FleetIntel record }\end{array}$ \\
\hline Existing Fleet Unit ID & EF_UnID & ID & $\begin{array}{l}\text { Unique identification code assigned to } \\
\text { each HS_EF_Unit record }\end{array}$ \\
\hline EUCG ID & EUCG_ID & ID & $\begin{array}{l}\text { Unique identification code assigned to } \\
\text { each record within the Electric Utility } \\
\text { Cost Group (EUCG) Database }\end{array}$ \\
\hline \multirow[t]{2}{*}{ hydroAMP ID } & hydroAMP_ID & ID & $\begin{array}{l}\text { Unique identification code assigned to } \\
\text { each record within the hydroAMP } \\
\text { Database }\end{array}$ \\
\hline & GADS_ID & ID & \\
\hline Ownership & PctOwn & PCT & Ownership \% \\
\hline Accumulated Depreciation & $\mathrm{AD}$ & $1,000 \mathrm{~s}$ of $\mathrm{LCU}$ & Accumulated depreciation \\
\hline Admin Direct Contracts & A1_C & $1,000 \mathrm{~s}$ of $\mathrm{LCU}$ & Admin 1-Direct contracts \\
\hline Admin Direct Labor & A1_L & $1,000 \mathrm{~s}$ of $\mathrm{LCU}$ & Admin 1-Direct labor \\
\hline Admin Direct Other & A1_O & $1,000 \mathrm{~s}$ of $\mathrm{LCU}$ & Admin 1-Direct other \\
\hline Admin Indirect Contracts & $\mathrm{A} 2 \_\mathrm{C}$ & 1,000 s of $\mathrm{LCU}$ & Admin 2-In-Direct contracts \\
\hline Admin Indirect Labor & A2_L & 1,000 s of $\mathrm{LCU}$ & Admin 2-In-Direct labor \\
\hline Admin Indirect Other & A2_O & 1,000 s of $\mathrm{LCU}$ & Admin 2-In-Direct other \\
\hline Capital Dam Contracts & I2DRG_C & $1,000 \mathrm{~s}$ of $\mathrm{LCU}$ & $\begin{array}{l}\text { Investment } 2 \text { - Capital } \\
\text { dam/reservoir/general contracts }\end{array}$ \\
\hline Capital Dam Labor & I2DRG_L & $1,000 \mathrm{~s}$ of $\mathrm{LCU}$ & $\begin{array}{l}\text { Investment } 2 \text { - Capital } \\
\text { dam/reservoir/general labor }\end{array}$ \\
\hline Capital Dam Other & I2DRG_O & $1,000 \mathrm{~s}$ of $\mathrm{LCU}$ & $\begin{array}{l}\text { Investment } 2 \text { - Capital } \\
\text { dam/reservoir/general other }\end{array}$ \\
\hline Capital Environ Contracts & I2EREG_C & $1,000 \mathrm{~s}$ of $\mathrm{LCU}$ & $\begin{array}{l}\text { Investment } 2-\text { Capital environmental } \\
\text { and regulatory contracts }\end{array}$ \\
\hline Capital Environ Labor & I2EREG_L & $1,000 \mathrm{~s}$ of $\mathrm{LCU}$ & $\begin{array}{l}\text { Investment } 2-\text { Capital environmental } \\
\text { and regulatory labor }\end{array}$ \\
\hline Capital Environ Other & I2EREG_O & $1,000 \mathrm{~s}$ of $\mathrm{LCU}$ & $\begin{array}{l}\text { Investment } 2-\text { Capital environmental } \\
\text { and regulatory other }\end{array}$ \\
\hline
\end{tabular}




\begin{tabular}{|c|c|c|c|}
\hline Capital Powerhouse Contracts & I2PWR_C & $1,000 \mathrm{~s}$ of $\mathrm{LCU}$ & $\begin{array}{l}\text { Investment 2-Capital powerhouse } \\
\text { contracts }\end{array}$ \\
\hline Capital Powerhouse Labor & I2PWR_L & $1,000 \mathrm{~s}$ of $\mathrm{LCU}$ & $\begin{array}{l}\text { Investment 2-Capital powerhouse } \\
\text { labor }\end{array}$ \\
\hline Capital Powerhouse Other & I2PWR_O & $1,000 \mathrm{~s}$ of $\mathrm{LCU}$ & $\begin{array}{l}\text { Investment 2-Capital powerhouse } \\
\text { other }\end{array}$ \\
\hline EnvironCulturalContracts & ER3_C & $1,000 \mathrm{~s}$ of $\mathrm{LCU}$ & $\begin{array}{l}\text { Environmental and regulatory } 3- \\
\text { Cultural contracts }\end{array}$ \\
\hline Environ Cultural Labor & ER3_L & $1,000 \mathrm{~s}$ of $\mathrm{LCU}$ & $\begin{array}{l}\text { Environmental and regulatory } 3 \text { - } \\
\text { Cultural labor }\end{array}$ \\
\hline Environ Cultural Other & ER3_O & $1,000 \mathrm{~s}$ of $\mathrm{LCU}$ & $\begin{array}{l}\text { Environmental and regulatory } 3 \text { - } \\
\text { Cultural other }\end{array}$ \\
\hline Environ Fish Contracts & ER1_C & $1,000 \mathrm{~s}$ of $\mathrm{LCU}$ & $\begin{array}{l}\text { Environmental and regulatory } 1 \text {-Fish } \\
\text { and wildlife contracts }\end{array}$ \\
\hline Environ Fish Labor & ER1_L & $1,000 \mathrm{~s}$ of $\mathrm{LCU}$ & $\begin{array}{l}\text { Environmental and regulatory } 1 \text {-Fish } \\
\text { and wildlife labor }\end{array}$ \\
\hline EnvironFishOther & ER1_O & $1,000 \mathrm{~s}$ of $\mathrm{LCU}$ & $\begin{array}{l}\text { Environmental and regulatory } 1 \text {-Fish } \\
\text { and wildlife other }\end{array}$ \\
\hline Environ Other Contracts & ER5_C & $1,000 \mathrm{~s}$ of $\mathrm{LCU}$ & $\begin{array}{l}\text { Environmental and Regulatory 5- } \\
\text { Other Contracts }\end{array}$ \\
\hline Environ Other Labor & ER5_L & $1,000 \mathrm{~s}$ of $\mathrm{LCU}$ & $\begin{array}{l}\text { Environmental and Regulatory 5- } \\
\text { Other Labor }\end{array}$ \\
\hline Environ Other Other & ER5_O & 1,000 s of $\mathrm{LCU}$ & $\begin{array}{l}\text { Environmental and Regulatory 5- } \\
\text { Other Other }\end{array}$ \\
\hline Environ Rec Contracts & ER2_C & $1,000 \mathrm{~s}$ of $\mathrm{LCU}$ & $\begin{array}{l}\text { Environmental and Regulatory 2- } \\
\text { Recreation Contracts }\end{array}$ \\
\hline Environ Rec Labor & ER2_L & $1,000 \mathrm{~s}$ of $\mathrm{LCU}$ & $\begin{array}{l}\text { Environmental and Regulatory 2- } \\
\text { Recreation Labor }\end{array}$ \\
\hline Environ Rec Other & ER2_O & $1,000 \mathrm{~s}$ of $\mathrm{LCU}$ & $\begin{array}{l}\text { Environmental and Regulatory 2- } \\
\text { Recreation Other }\end{array}$ \\
\hline Environ Safety Contracts & ER4_C & $1,000 \mathrm{~s}$ of $\mathrm{LCU}$ & $\begin{array}{l}\text { Environmental and Regulatory 4- } \\
\text { Dam and Public Safety Contracts }\end{array}$ \\
\hline EnvironSafetyLabor & ER4_L & $1,000 \mathrm{~s}$ of $\mathrm{LCU}$ & $\begin{array}{l}\text { Environmental and Regulatory 4- } \\
\text { Dam and Public Safety Labor }\end{array}$ \\
\hline Environ Safety Other & ER4_O & $1,000 \mathrm{~s}$ of $\mathrm{LCU}$ & $\begin{array}{l}\text { Environmental and Regulatory 4- } \\
\text { Dam and Public Safety Other }\end{array}$ \\
\hline
\end{tabular}




\begin{tabular}{|c|c|c|c|}
\hline Fees Land Contracts & LW1_C & $1,000 \mathrm{~s}$ of $\mathrm{LCU}$ & $\begin{array}{l}\text { Land and Water Fees } 1 \text {-Land } \\
\text { Rentals/Fees Contracts }\end{array}$ \\
\hline Fees Land Labor & LW1_L & $1,000 \mathrm{~s}$ of $\mathrm{LCU}$ & $\begin{array}{l}\text { Land and Water Fees } 1 \text { - Land } \\
\text { Rentals/Fees Labor }\end{array}$ \\
\hline Fees Land Other & LW1_O & $1,000 \mathrm{~s}$ of $\mathrm{LCU}$ & $\begin{array}{l}\text { Land and Water Fees 1-Land } \\
\text { Rentals/Fees Other }\end{array}$ \\
\hline Fees Water Contracts & LW2_C & $1,000 \mathrm{~s}$ of $\mathrm{LCU}$ & $\begin{array}{l}\text { Land and Water Fees 2-Water } \\
\text { Rentals/Fees Contracts }\end{array}$ \\
\hline FeesWaterLabor & LW2_L & 1,000 s of $\mathrm{LCU}$ & $\begin{array}{l}\text { Land and Water Fees 2-Water } \\
\text { Rentals/Fees Labor }\end{array}$ \\
\hline Fees Water Other & LW2_O & $1,000 \mathrm{~s}$ of $\mathrm{LCU}$ & $\begin{array}{l}\text { Land and Water Fees 2-Water } \\
\text { Rentals/Fees Other }\end{array}$ \\
\hline Fixed Asset & FAV & $1,000 \mathrm{~s}$ of $\mathrm{LCU}$ & Fixed asset value (undepreciated) \\
\hline Invest Dam Contracts & I1DRG_C & $1,000 \mathrm{~s}$ of $\mathrm{LCU}$ & $\begin{array}{l}\text { Investment } 1 \text {-major expense- } \\
\text { nonrecurring dam/reservoir/general } \\
\text { contracts }\end{array}$ \\
\hline Invest Dam Labor & I1DRG_L & $1,000 \mathrm{~s}$ of $\mathrm{LCU}$ & $\begin{array}{l}\text { Investment } 1 \text {-Major expense- } \\
\text { Nonrecurring dam/reservoir/general } \\
\text { labor }\end{array}$ \\
\hline Invest Dam Other & I1DRG_O & $1,000 \mathrm{~s}$ of $\mathrm{LCU}$ & $\begin{array}{l}\text { Investment } 1 \text {-Major expense- } \\
\text { Nonrecurring dam/reservoir/general } \\
\text { other }\end{array}$ \\
\hline Invest Environ Contracts & I1EREG_C & $1,000 \mathrm{~s}$ of $\mathrm{LCU}$ & $\begin{array}{l}\text { Investment } 1-\text { Major expense- } \\
\text { Nonrecurring environmental and } \\
\text { regulatory contracts }\end{array}$ \\
\hline Invest Environ Labor & I1EREG_L & $1,000 \mathrm{~s}$ of $\mathrm{LCU}$ & $\begin{array}{l}\text { Investment } 1-\text { Major Expense- } \\
\text { Nonrecurring environmental and } \\
\text { regulatory and labor }\end{array}$ \\
\hline Invest Environ Other & I1EREG_O & $1,000 \mathrm{~s}$ of $\mathrm{LCU}$ & $\begin{array}{l}\text { Investment } 1-\text { Major Expense- } \\
\text { Nonrecurring environmental and } \\
\text { regulatory other }\end{array}$ \\
\hline Invest Powerhouse Contracts & I1PWR_C & $1,000 \mathrm{~s}$ of $\mathrm{LCU}$ & $\begin{array}{l}\text { Investment } 1-\text { Major expense- } \\
\text { Nonrecurring powerhouse contracts }\end{array}$ \\
\hline InvestPowerhouseLabor & I1PWR_L & $1,000 \mathrm{~s}$ of $\mathrm{LCU}$ & $\begin{array}{l}\text { Investment } 1 \text { - Major expense- } \\
\text { Nonrecurring powerhouse labor }\end{array}$ \\
\hline Invest Powerhouse Other & I1PWR_O & $1,000 \mathrm{~s}$ of $\mathrm{LCU}$ & $\begin{array}{l}\text { Investment } 1-\text { Major expense- } \\
\text { Nonrecurring powerhouse other }\end{array}$ \\
\hline Maint Non-Powerhouse Contracts & M2_C & $1,000 \mathrm{~s}$ of $\mathrm{LCU}$ & $\begin{array}{l}\text { Maintenance } 2-\text { Nonpowerhouse } \\
\text { maintenance expense Contracts }\end{array}$ \\
\hline
\end{tabular}




\begin{tabular}{|c|c|c|c|}
\hline Maint Non-PowerhouseLabor & M2_L & 1,000 s of $\mathrm{LCU}$ & $\begin{array}{l}\text { Maintenance } 2 \text {-Nonpowerhouse } \\
\text { maintenance expense labor }\end{array}$ \\
\hline Maint Non-Powerhouse Other & $\mathrm{M} 2 \_\mathrm{O}$ & $1,000 \mathrm{~s}$ of $\mathrm{LCU}$ & $\begin{array}{l}\text { Maintenance } 2 \text {-Nonpowerhouse } \\
\text { maintenance expense other }\end{array}$ \\
\hline Maint Powerhouse Contracts & M1_C & $1,000 \mathrm{~s}$ of $\mathrm{LCU}$ & $\begin{array}{l}\text { Maintenance } 1 \text { - Powerhouse } \\
\text { maintenance expense contracts }\end{array}$ \\
\hline Maint Powerhouse Labor & M1_L & $1,000 \mathrm{~s}$ of $\mathrm{LCU}$ & $\begin{array}{l}\text { Maintenance } 1 \text {-Powerhouse } \\
\text { maintenance expense labor }\end{array}$ \\
\hline Maint Powerhouse Other & M1_O & $1,000 \mathrm{~s}$ of $\mathrm{LCU}$ & $\begin{array}{l}\text { Maintenance } 1 \text {-Powerhouse } \\
\text { maintenance expense other }\end{array}$ \\
\hline New Capital Dam Contracts & I3DRG_C & $1,000 \mathrm{~s}$ of $\mathrm{LCU}$ & $\begin{array}{l}\text { Investment } 3 \text { - Capital } \\
\text { dam/reservoir/general contracts }\end{array}$ \\
\hline New Capital Dam Labor & I3DRG_L & $1,000 \mathrm{~s}$ of $\mathrm{LCU}$ & $\begin{array}{l}\text { Investment } 3 \text {-Capital } \\
\text { dam/reservoir/general labor }\end{array}$ \\
\hline New Capital Dam Other & I3DRG_O & $1,000 \mathrm{~s}$ of $\mathrm{LCU}$ & $\begin{array}{l}\text { Investment } 3 \text { - Capital } \\
\text { dam/reservoir/general other }\end{array}$ \\
\hline NewCapitalEnvironContracts & I3EREG_C & $1,000 \mathrm{~s}$ of $\mathrm{LCU}$ & $\begin{array}{l}\text { Investment } 3 \text { - Capital environmental } \\
\text { and regulatory contracts }\end{array}$ \\
\hline New Capital Environ Labor & I3EREG_L & $1,000 \mathrm{~s}$ of $\mathrm{LCU}$ & $\begin{array}{l}\text { Investment } 3 \text { - Capital environmental } \\
\text { and regulatory labor }\end{array}$ \\
\hline New Capital Environ Other & I3EREG_O & $1,000 \mathrm{~s}$ of $\mathrm{LCU}$ & $\begin{array}{l}\text { Investment } 3 \text { - Capital environmental } \\
\text { and regulatory other }\end{array}$ \\
\hline \multicolumn{2}{|c|}{ New Capital Powerhouse Contracts I3PWR_C } & 1,000 s of $\mathrm{LCU}$ & $\begin{array}{l}\text { Investment 3-Capital powerhouse } \\
\text { contracts }\end{array}$ \\
\hline New Capital Powerhouse Labor & I3PWR_L & 1,000 s of $\mathrm{LCU}$ & $\begin{array}{l}\text { Investment 3-Capital powerhouse } \\
\text { labor }\end{array}$ \\
\hline New Capital Powerhouse Other & I3PWR_O & $1,000 \mathrm{~s}$ of $\mathrm{LCU}$ & $\begin{array}{l}\text { Investment } 3 \text {-Capital powerhouse } \\
\text { other }\end{array}$ \\
\hline Ops Facilities Contracts & $\mathrm{O} 1 \_\mathrm{C}$ & 1,000 s of $\mathrm{LCU}$ & $\begin{array}{l}\text { Operations } 1 \text { facilities operations } \\
\text { contracts }\end{array}$ \\
\hline Ops Facilities Labor & O1_L & $1,000 \mathrm{~s}$ of $\mathrm{LCU}$ & Operations 1 facilities operations labor \\
\hline Ops Facilities Other & O1_O & $1,000 \mathrm{~s}$ of $\mathrm{LCU}$ & Operations 1 facilities operations other \\
\hline Ops Generation Contracts & $\mathrm{O} 2 \_\mathrm{C}$ & 1,000 s of $\mathrm{LCU}$ & $\begin{array}{l}\text { Operations } 2-\text { Generation planning } \\
\text { and integration contracts }\end{array}$ \\
\hline Ops Generation Labor & O2_L & 1,000 s of $\mathrm{LCU}$ & $\begin{array}{l}\text { Operations } 2-\text { Generation planning } \\
\text { and integration labor }\end{array}$ \\
\hline
\end{tabular}




\begin{tabular}{|c|c|c|c|}
\hline Ops Generation Other & $\mathrm{O} 2 \_\mathrm{O}$ & $1,000 \mathrm{~s}$ of $\mathrm{LCU}$ & $\begin{array}{l}\text { Operations } 2 \text { - Generation planning } \\
\text { and integration other }\end{array}$ \\
\hline OpsMiscContracts & $\mathrm{O} 3 \_\mathrm{C}$ & $1,000 \mathrm{~s}$ of $\mathrm{LCU}$ & $\begin{array}{l}\text { Operations 3-miscellaneous } \\
\text { contracts }\end{array}$ \\
\hline Ops Misc Labor & O3_L & 1,000 s of $\mathrm{LCU}$ & Operations 3 -miscellaneous labor \\
\hline Ops Misc Other & O3_O & $1,000 \mathrm{~s}$ of $\mathrm{LCU}$ & Operations 3-miscellaneous other \\
\hline Water Spilled & SPILL & 1000 acre-ft & Water spilled \\
\hline Exciter Current Rating & EXI & $\mathrm{A}$ & Rating of the exciter \\
\hline Generator Current Rating & GENI & A & Rating of the generator \\
\hline FullCapacity & Full_Cap & $\mathrm{cfs}$ & Full capacity \\
\hline Operating Head & Oper_Head & $\mathrm{ft}$ & Plant design head \\
\hline Head & TURB_HEAD & $\mathrm{ft}$ & Turbine rated head \\
\hline Event End Time & EventETime & hh:mm & Hour and minute event ends \\
\hline Event Start Time & EventSTime & hh:mm & $\begin{array}{l}\text { Hour and minute event starts 24:00 } \\
\text { clock }\end{array}$ \\
\hline CondensingHours & $\mathrm{CH}$ & Hours & Condensing hours \\
\hline Condensing Hours & $\mathrm{UCH}$ & Hours & Condensing hours unit level \\
\hline ForcedOutageHours & $\mathrm{FOH}$ & Hours & Forced outage hours \\
\hline Forced Outage Hours & UFOH & Hours & Total forced outage hours unit level \\
\hline MaintenanceOutageHours & $\mathrm{MOH}$ & Hours & Maintenance outage hours \\
\hline Maintenance Outage Hours & $\mathrm{UMOH}$ & Hours & $\begin{array}{l}\text { Total maintenance outage hours unit } \\
\text { level }\end{array}$ \\
\hline $\mathrm{OMC} \mathrm{FOH}$ & OMC_FOH & Hours & $\mathrm{OMC} \mathrm{FOH}$ \\
\hline OMCMOH & OMC_MOH & Hours & $\mathrm{OMC} \mathrm{MOH}$ \\
\hline OMC POH & OMC_POH & Hours & $\mathrm{OMC} \mathrm{POH}$ \\
\hline Period Hours & $\mathrm{PH}$ & Hours & Period hours \\
\hline Period Hours & UPH & Hours & Period hours unit level \\
\hline PlannedOutageHours & $\mathrm{POH}$ & Hours & Planned outage hours \\
\hline Planned Outage Hours & UPOH & Hours & Total planned outage hours unit level \\
\hline PumpingHours & $\mathrm{PuH}$ & Hours & Pumping hours \\
\hline Pumping Hours & $\mathrm{UPuH}$ & Hours & Pumping hours unit level \\
\hline
\end{tabular}




\begin{tabular}{|c|c|c|c|}
\hline ReserveShutdownHours & RSH & Hours & Reserve shutdown hours \\
\hline Reserve Shutdown Hours & URSH & Hours & Reserve shutdown hours unit level \\
\hline Service Hours & $\mathrm{SH}$ & Hours & Service hours \\
\hline Service Hours & USH & Hours & Service hours unit level \\
\hline Total Available Hours & TAH & Hours & $\begin{array}{l}\text { Sum of service, reserve shutdown, } \\
\text { pumping, and condensing }\end{array}$ \\
\hline Total Unavailable Hours & TUH & Hours & $\begin{array}{l}\text { Sum of planned, forced, and } \\
\text { maintenance outage hours }\end{array}$ \\
\hline Turbine Rated Capacity & TURB_CAP & hp & Rating of the turbine \\
\hline hydroAMP Record Number & HP_NUM & Integer & $\begin{array}{l}\text { Asset number in hydroAMP, started } \\
\text { from 1, will keep increasing }\end{array}$ \\
\hline Circuit Breaker Current Rating & $\mathrm{CBI}$ & $\mathrm{kA}$ & Rating of the circuit breaker \\
\hline Interrupt Rating & CB_INT & $\mathrm{kA}$ & Circuit breaker interrupt rating \\
\hline Circuit Breaker Voltage Rating & $\mathrm{CBV}$ & $\mathrm{kV}$ & Rating of the circuit breaker \\
\hline Diameter & TURB_D & $\mathrm{m}$ & Turbine diameter \\
\hline Event End Date & EventEDate & mm/dd/yyyy & Month, day, and year event ends \\
\hline Event Start Date & EventSDate & mm/dd/yyyy & Month, day, and year event starts \\
\hline Last Assessment Date & FRESH & mm/dd/yyyy & $\begin{array}{l}\text { Date of the most recent Tier } 1 \text { score } \\
\text { available for the component }\end{array}$ \\
\hline Tier2 Assessment Date & T2_DATE & mm/dd/yyyy & Date of the Tier 2 assessment \\
\hline Generator Stator Rated Capacity & GEN_CAP & MVA & Rating of the generator stator \\
\hline Control MW & Cont_MW & MW & MW per control room \\
\hline Gross Dependable Capacity & UGDC & MW & Hard capacity unit level \\
\hline Gross Max Capacity & UGMC & MW & Max generating capacity unit level \\
\hline Gross Capacity & Gross_MW & MW & Gross capacity \\
\hline Net Dependable Capacity & UNDC & MW & $\begin{array}{l}2 \% \text { difference between gross and net } \\
\text { values unit level }\end{array}$ \\
\hline Net Max Capacity & UNMC & MW & $\begin{array}{l}2 \% \text { difference between gross and net } \\
\text { values unit level }\end{array}$ \\
\hline Net Capacity & Net_MW & MW & Net capacity \\
\hline Unit capacity & UNITCAP & MW & Capacity of installed unit \\
\hline GrossActualGeneration & GAG & MWh & Gross actual generation \\
\hline
\end{tabular}




\begin{tabular}{|c|c|c|c|}
\hline Gross Actual Generation & UGAG & $\mathrm{MWh}$ & Gross actual generation \\
\hline NetActualGeneration & NAG & MWh & Net actual generation \\
\hline Net Actual Generation & UNAG & MWh & Net actual generation \\
\hline Power Factor & $\mathrm{PF}$ & N/A & Ratio of real power to apparent power \\
\hline Station Index & SI & N/A & $\begin{array}{l}\text { Score from } 0 \text { to } 10 \text { of plant health } \\
\text { taken as an average of unit index } \\
\text { ratings }\end{array}$ \\
\hline Speed & TURB_SPEED & $\mathrm{rpm}$ & Turbine synchronous speed \\
\hline Approved By & APPR & String & Managerial approver's name \\
\hline Battery Type & BATT_ $_{-}$ & String & $\begin{array}{l}\text { Vented Lead Acid (VLA) or Valve } \\
\text { Regulated Lead Acid (VRLA) }\end{array}$ \\
\hline Crane Type & CRANE & String & Overhead, gantry, monorail hoist \\
\hline DamType & Dam_Type & String & $\begin{array}{l}\text { Dam type: arch, buttress, concrete, } \\
\text { earthen, rock-filled, gravity, multi- } \\
\text { arch, roller-compacted-concrete, } \\
\text { masonry, timber crib, other }\end{array}$ \\
\hline Emergency Closure Gate Types & ECG & String & $\begin{array}{l}\text { Roller mounted, ring seal, wheel } \\
\text { mounted, cylinder, fixed wheel, } \\
\text { coaster, ring follower }\end{array}$ \\
\hline Emergency Closure Valve Types & EGV & String & Butterfly, spherical, cone \\
\hline Equipment Name & EQUIP & String & Turbine, transformer, crane, etc. \\
\hline Evaluated By & EVAL & String & Evaluator's name \\
\hline Excitation System Types & ES & String & Digital, analog, mechanical \\
\hline Excitation System Types & EXC_TYPE & String & Analog, digital or mechanical systems \\
\hline Generator Manufacturer & GEN_MANUF & String & Generator manufacturer \\
\hline Generator Manufacturer Group & GEN_MG & String & Generator manufacturer group name \\
\hline Governor Types & GOV & String & Digital, analog, mechanical \\
\hline Insulation & SWI & String & $\begin{array}{l}\text { Insulation material of the stator } \\
\text { winding }\end{array}$ \\
\hline OperatingPurpose & Purp & String & $\begin{array}{l}\text { Operating purpose: environmental, } \\
\text { flood control, generation, irrigation, } \\
\text { navigation, other }\end{array}$ \\
\hline
\end{tabular}




\begin{tabular}{|c|c|c|c|}
\hline Outage/Derating Event Type & EventType & String & $\begin{array}{l}\text { Planned, forced (U1, U2, U3), } \\
\text { maintenance, derates (PD, FD, D1, } \\
\text { D2, D3), startup failure, outage } \\
\text { extensions }\end{array}$ \\
\hline Owner name & OWN_NAME & String & Plant owner name \\
\hline Plant name & PLT_NAME & String & Name of plant \\
\hline ProductionMode & Prod_mode & String & $\begin{array}{l}\text { Production mode: base load, } \\
\text { intermediate, peaking, other }\end{array}$ \\
\hline Rehabilitation Contractor & CONT_REHAB & String & $\begin{array}{l}\text { If rehab work is performed on the } \\
\text { component, name of contractor }\end{array}$ \\
\hline Surge Arrestor Type & SA & String & Silicon carbide, metal oxide, varistor \\
\hline Tap Changer & TTP & String & Transformer tap changer \\
\hline Tier 1 Comments & T1_COM & String & Evaluator's comments \\
\hline Tier 2 Comments & $\mathrm{T} 2 \_\mathrm{COM}$ & String & Evaluator's comments \\
\hline Turbine Manufacturer Group & $\mathrm{T} \_\mathrm{MG}$ & String & Turbine manufacturer group name \\
\hline Turbine Type & TUR_TYPE & String & $\begin{array}{l}\text { Turbine drive type: Francis, Kaplan, } \\
\text { Pelton, Propeller }\end{array}$ \\
\hline TurbineType & Turbine_Type & String & Predominate turbine runner type \\
\hline Unit Manufacturer & MANUF & String & Turbine manufacturer \\
\hline Unit Name & UNITNAME & String & Name of units \\
\hline Utility Name & UTIL & String & Name of ownership company \\
\hline Crane Rated Capacity & CRANE_tons & Tons & Rating of the crane \\
\hline Gate Height & GATE_H & Unknown & Dimensions of the gate \\
\hline Gate Weight & GATE_Weight & Unknown & Dimensions of the gate \\
\hline Gate Width & GATE_W & Unknown & Dimensions of the gate \\
\hline Pressure & GATE_P & Unknown & $\begin{array}{l}\text { Not a single component has input a } \\
\text { pressure value }\end{array}$ \\
\hline Exciter Voltage Rating & EXV & $\mathrm{V}$ & Rating of the exciter \\
\hline Generator Voltage Rating & GENV & $\mathrm{V}$ & Rating of the generator \\
\hline DO Enhancement & DO_Enhn & $\mathrm{Y} / \mathrm{N}$ & Dissolved oxygen (DO) enhancement \\
\hline Fish Habitat & Fish_Hab & $\mathrm{Y} / \mathrm{N}$ & Fish habitat improvement \\
\hline Flood Control & Fld_Cont & $\mathrm{Y} / \mathrm{N}$ & Flood control \\
\hline
\end{tabular}




\begin{tabular}{|c|c|c|c|}
\hline Flow Restrictions & Flow_Rest & $\mathrm{Y} / \mathrm{N}$ & Flow restrictions \\
\hline Oil Containment & Oil_Cont & $\mathrm{Y} / \mathrm{N}$ & Oil containment \\
\hline Plant Project & Plant_Prj & $\mathrm{Y} / \mathrm{N}$ & $\begin{array}{l}\text { Plant project, run of river(Y) vs. } \\
\text { storage }(\mathrm{N}) \text { : } \mathrm{MOP} \text { is more descriptive }\end{array}$ \\
\hline Powerhouse & Pwr_House & $\mathrm{Y} / \mathrm{N}$ & Enclosed powerhouse \\
\hline Recreational Constraints & Recre_Const & $\mathrm{Y} / \mathrm{N}$ & Recreational constraints \\
\hline Spare $\mathrm{Y} / \mathrm{N}$ & SPARE & $\mathrm{Y} / \mathrm{N}$ & Is this component an onsite spare \\
\hline Temperature Restrictions & Temp_Rest & $\mathrm{Y} / \mathrm{N}$ & Temperature restrictions \\
\hline Max Commercial OpYear & Max_Com_Op_Yr & year & Date of commercial operation \\
\hline In Service Year & CISY & уууу & Component specific in service year \\
\hline Active Units & Actv_U & & Active units \\
\hline Actual Unit Starts & AcUS & & Count of actual unit starts \\
\hline Adjusted Weighted Score & ADJTIER1 & & $\begin{array}{l}\text { Revised Tier } 1 \text { score accounting for } \\
\text { Tier } 2 \text { adjustment }\end{array}$ \\
\hline AGCUnits & AGC_U & & Automatic generation control units \\
\hline Asset Count & ASSET_COUNT & & Number of assets reported per unit \\
\hline Attempted Unit Starts & AtUS & & Count of unit starts attempted \\
\hline Cause Code & Cause_Code & & NERC designated cause code \\
\hline CI 1 & HCI1 & & Condition indicator 1 age \\
\hline CI 2 & HCI2 & & $\begin{array}{l}\text { Condition indicator } 2 \text { physical } \\
\text { condition }\end{array}$ \\
\hline CI 3 & $\mathrm{HCI} 3$ & & Condition indicator 3 operations \\
\hline CI 4 & HCI4 & & Condition indicator 4 maintenance \\
\hline CI 5 & HCI5 & & Condition indicator 5 other \\
\hline Condense Units & Cond_U & & Condense units \\
\hline Control Plants & Cont_Plants & & No. of plants per control room \\
\hline Control Staff & Cont_Staff & & Unstaffed or staffed control room \\
\hline Control Units & Cont_Units & & No. of units per control room \\
\hline Data Quality Indicator & DQI & & $\begin{array}{l}\text { Score representing how recently the } \\
\text { component score has been updated }\end{array}$ \\
\hline hydroAMP Plant Code & HP_CODE & & Hydroamp specific plant code \\
\hline
\end{tabular}




\begin{tabular}{|c|c|c|}
\hline Impulse Level & ECV_IMP & $\begin{array}{l}\text { Emergency closure valve impulse } \\
\text { level }\end{array}$ \\
\hline Number of Cells & BATT_CELLS & Number of cells in battery \\
\hline Number of Phases & PHASES & Exciter number of phases \\
\hline Number of Poles & POLES & Number of rotor poles \\
\hline Number of Windings & SW_TURNS & Number of stator winding turns \\
\hline NumberUnits & \#_U & Number units \\
\hline Operator & OPS & Gate closure operator \\
\hline PlantType & Pl_type & Plant type \\
\hline RegionCode & Reg_Code & Region code from EUCG \\
\hline RemoteUnits & Rmt_U & Remote units \\
\hline River & River & River \\
\hline State & State & State \\
\hline Status & Status & Status \\
\hline Tier 1 Score & TIER1 & $\begin{array}{l}\text { Score from } 0 \text { to } 10 \text { of unit health taken } \\
\text { as a weighted average of condition } \\
\text { index }(\mathrm{CI})\end{array}$ \\
\hline Tier2 Adjustment & TIER2ADJ & $\begin{array}{l}\text { Tier } 2 \text { scores adjust the Tier } 1 \text { scores } \\
\text { up or down }\end{array}$ \\
\hline Unit Number & U_NUM & Placement within powerhouse \\
\hline Weighting Factor 1 & HWF1 & Weighting factor 1 age \\
\hline Weighting Factor 2 & HWF2 & Weighting factor 2 physical condition \\
\hline Weighting Factor 3 & HWF3 & Weighting factor 3 operations \\
\hline Weighting Factor 4 & HWF4 & Weighting factor 4 maintenance \\
\hline Weighting Factor 5 & HWF5 & Weighting factor 5 other \\
\hline
\end{tabular}




\section{GEOSPATIAL HYDROLOGIC}

HSDM: HS_DG: Summary of geospatial hydrologic data components

\begin{tabular}{llll}
\hline \multicolumn{1}{c}{ Data_Component } & \multicolumn{1}{c}{ Alias } & \multicolumn{1}{c}{ Type } & \multicolumn{1}{c}{ Description } \\
\hline HS_m_ResFlowline & $\begin{array}{l}\text { NHDPlus V1 Common } \\
\text { Identifier }\end{array}$ & ID & NHDPlus V1 flowline common identifier. \\
HS_HUC_8 & $\begin{array}{l}\text { HydroSource HUC-8 } \\
\text { Geospatial Polygon }\end{array}$ & $\begin{array}{l}\text { Geospatial } \\
\text { polyline layer }\end{array}$ & HydroSource HUC-8 geospatial polygon \\
HS_HUC_6 & $\begin{array}{l}\text { HydroSource HUC-6 } \\
\text { Geospatial Polygon }\end{array}$ & $\begin{array}{l}\text { Geospatial } \\
\text { polyline layer }\end{array}$ & HydroSource HUC-6 geospatial polygon \\
HS_HUC_2 & $\begin{array}{l}\text { HydroSource HUC-2 } \\
\text { Geospatial Polygon }\end{array}$ & $\begin{array}{l}\text { Geospatial } \\
\text { polyline layer }\end{array}$ & HydroSource HUC-2 geospatial polygon \\
HS_EF_RelationalTbl & $\begin{array}{l}\text { HydroSource Relational } \\
\text { Table }\end{array}$ & $\begin{array}{l}\text { Geospatial } \\
\text { polyline layer }\end{array}$ & $\begin{array}{l}\text { HydroSource relational table between the } \\
\text { HS_EF_Plant and HS_EF_Reservoir layers }\end{array}$ \\
\hline
\end{tabular}

HS_m_ResFlowline: Attribute summary

\begin{tabular}{|c|c|c|c|c|}
\hline & Field Name & Alias & Unit & Description \\
\hline 1 & COMID & NHDPlus V1 Common Identifier & ID & NHDPlus V1 flowline common identifier \\
\hline
\end{tabular}

HS_HUC_8: Attribute summary

\begin{tabular}{ccccc}
\hline & Field Name & Alias & Unit & Description \\
\hline 1 & HUC_8 & 8-Digit HUC & ID & Eight-digit USGS HUC
\end{tabular}

HS_HUC_6: Attribute summary

\begin{tabular}{cccc}
\hline Field Name & Alias & Unit & Description \\
\hline HUC_6 & 6-Digit HUC & ID & Six-digit USGS HUC \\
\hline
\end{tabular}

HS_HUC_2: Attribute summary

\begin{tabular}{cccc}
\hline Field Name & Alias & Unit & Description \\
HUC_2 & 2-Digit HUC & ID & Two-digit USGS HUC \\
\hline
\end{tabular}


HS_EF_RelationalTbl: Attribute summary

\begin{tabular}{llll}
\hline Field Name & \multicolumn{1}{c}{ Alias } & Unit & \multicolumn{1}{c}{ Description } \\
\hline EF_RID & $\begin{array}{l}\text { Existing Fleet } \\
\text { Relational ID }\end{array}$ & ID & $\begin{array}{l}\text { Unique identification code enabling a many-to-many relationship } \\
\text { between the HS_EF_Plant and HS_EF_Reservoir layers }\end{array}$ \\
EF_PtID & $\begin{array}{l}\text { Existing Fleet Plant } \\
\text { ID }\end{array}$ & ID & Unique identification code assigned to each HS_EF_Plant record \\
EF_RsID & $\begin{array}{l}\text { Existing Fleet } \\
\text { Reservoir ID }\end{array}$ & ID & Unique identification code assigned to each HS_EF_Reservoir record \\
\hline
\end{tabular}

\section{HYDROPOWER MARKET REPORT}

Summary of Hydropower Market Report (HMR) Data Components

\begin{tabular}{|c|c|c|c|}
\hline Data_Component & Alias & Type & Description \\
\hline HS_MonthlyGen & $\begin{array}{l}\text { Monthly Hydropower } \\
\text { Generation }\end{array}$ & Time series & $\begin{array}{l}\text { Monthly hydropower generation of } \\
\text { preoperational, operational, and retired } \\
\text { hydropower plants in the United States }\end{array}$ \\
\hline HS_RefurbUpgrade & $\begin{array}{l}\text { Refurbishments and } \\
\text { Upgrades }\end{array}$ & $\begin{array}{l}\text { Nonspatial } \\
\text { table }\end{array}$ & $\begin{array}{l}\text { Data integrated from the IIR database on } \\
\text { refurbishment and upgrade (R\&U) investment } \\
\text { projects }\end{array}$ \\
\hline HS_MWChange & $\begin{array}{l}\text { Existing Fleet Annual } \\
\text { Capacity Changes }\end{array}$ & Time series & $\begin{array}{l}\text { Annual hydropower plant capacity changes } \\
\text { from the EIA form } 860\end{array}$ \\
\hline HS_PPA & $\begin{array}{l}\text { Power Purchase } \\
\text { Agreements }\end{array}$ & $\begin{array}{l}\text { Nonspatial } \\
\text { table }\end{array}$ & $\begin{array}{l}\text { Data integrated from FERC's Form } 1 \text { data on } \\
\text { power purchase transactions between electric } \\
\text { utilities and hydropower plant owners }\end{array}$ \\
\hline
\end{tabular}

HS_MonthlyGen: Attribute summary

\begin{tabular}{|c|c|c|c|}
\hline Alias & Field Name & Unit & Description \\
\hline HS_MonthlyGen ID & MoGenID & ID & $\begin{array}{l}\text { Unique identification code assigned to each } \\
\text { HS_MonthlyGen record }\end{array}$ \\
\hline Existing Fleet Plant ID & EF_PtID & ID & $\begin{array}{l}\text { Unique identification code assigned to each HS_EF_Plant } \\
\text { record }\end{array}$ \\
\hline EIA Plant ID & EIA_PtID & ID & Plant-level ID for data from the EIA \\
\hline Generation Year and Month & Date & уууутm & $\begin{array}{l}\text { Year and month that hydropower generation and } \\
\text { consumption reported by the EIA occurred }\end{array}$ \\
\hline Plant Name & Pt_Nm & $\mathrm{N} / \mathrm{A}$ & Plant name \\
\hline Hydropower Net Generation & HYNetGen & $\mathrm{MWh}$ & $\begin{array}{l}\text { Monthly net hydropower generation of a hydropower } \\
\text { plant }\end{array}$ \\
\hline
\end{tabular}




$\begin{array}{llll}\begin{array}{l}\text { Pumped Storage Net } \\ \text { Generation }\end{array} & \text { PSNetGen } & \text { MWh } & \begin{array}{l}\text { Monthly net pumped storage generation of a hydropower } \\ \text { plant }\end{array} \\ \begin{array}{l}\text { Pumped Storage } \\ \text { Consumption }\end{array} & \text { PSConsum } & \text { MWh } & \begin{array}{l}\text { Monthly pumped storage consumption of a hydropower } \\ \text { plant }\end{array} \\ \begin{array}{l}\text { Pumped Storage Gross } \\ \text { Generation }\end{array} & \text { PSGross } & \text { MWh } & \begin{array}{l}\text { Monthly gross pumped storage generation of a } \\ \text { hydropower plant }\end{array}\end{array}$

HS_RefurbUpgrade: Attribute summary

\begin{tabular}{|c|c|c|c|}
\hline Alias & Field Name & Unit & Description \\
\hline $\begin{array}{l}\text { Plant Refurb and } \\
\text { Upgrade ID }\end{array}$ & Pt_RUID & ID & $\begin{array}{l}\text { Unique identification code assigned to each } \\
\text { HS_RefurbUpgrade record }\end{array}$ \\
\hline Existing Fleet Plant ID & EF_PtID & ID & $\begin{array}{l}\text { Unique identification code assigned to each } \\
\text { HS_EF_Plant record }\end{array}$ \\
\hline IIR Plant ID & IIR_PtID & ID & $\begin{array}{l}\text { Unique identification code assigned to each plant-level } \\
\text { record within the IIR Database }\end{array}$ \\
\hline Project name & PRJ_NAME & N/A & $R \& U$ project name \\
\hline Owner name & OWN_NAME & $\mathrm{N} / \mathrm{A}$ & Plant owner name \\
\hline Plant name & PLT_NAME & N/A & Name of plant undergoing $R \& U$ \\
\hline Plant state & PLT_STATE & $\mathrm{N} / \mathrm{A}$ & Plant location (state) \\
\hline Unit name & UNITNAME & $\mathrm{N} / \mathrm{A}$ & Name of units undergoing $R \& U$ \\
\hline Project type & PRJ_TYPE & N/A & $\mathrm{R} \& \mathrm{U}$ project type \\
\hline Project kickoff & PRJ_KOFF & $\mathrm{N} / \mathrm{A}$ & $\mathrm{R} \& \mathrm{U}$ project kickoff year \\
\hline Project status & PRJ_STAT & N/A & $\mathrm{R} \& \mathrm{U}$ project status \\
\hline $\begin{array}{l}\text { Project total investment } \\
\text { value }\end{array}$ & tPRJ_TIV & $\begin{array}{l}\text { Million } \\
(2017 \$)\end{array}$ & Project total investment value \\
\hline Project scope & SCOPE & N/A & $\mathrm{R} \& \mathrm{U}$ detailed project description \\
\hline Turbine work & TURBINE & $\mathrm{N} / \mathrm{A}$ & $\begin{array}{l}1 \text { if the project scope involves turbine modifications; } 0 \\
\text { otherwise }\end{array}$ \\
\hline Generator work & GNRATOR & $\mathrm{N} / \mathrm{A}$ & $\begin{array}{l}1 \text { if the project scope involves generator modifications; } 0 \\
\text { otherwise }\end{array}$ \\
\hline
\end{tabular}


HS_MWChange: Attribute summary

\begin{tabular}{|c|c|c|c|}
\hline Alias & Field Name & Unit & Description \\
\hline HS_MWChange ID & MWChngID & ID & $\begin{array}{l}\text { Annual hydropower plant capacity changes from EIA form } \\
860\end{array}$ \\
\hline Existing Fleet Plant ID & EF_PtID & ID & $\begin{array}{l}\text { Unique identification code assigned to each HS_EF_Plant } \\
\text { record }\end{array}$ \\
\hline EIA Plant ID & EIA_PtID & ID & Plant-level ID for data from the EIA \\
\hline EIA Form 860 Year & EIA_Year & N/A & $\begin{array}{l}\text { Year in which the capacity change was reported in EIA } \\
\text { Form } 860\end{array}$ \\
\hline HMR Region & HMR_Regn & N/A & Region where plant is located \\
\hline Change in Plant Capacity & MWChange & MW & Change in plant capacity \\
\hline Capacity Change Category & Category & N/A & $\begin{array}{l}\text { Capacity change category: Capacity addition, downrate, } \\
\text { retirement }\end{array}$ \\
\hline
\end{tabular}

HS_PPA: Attribute summary

\begin{tabular}{|c|c|c|c|}
\hline Alias & Field Name & Unit & Description \\
\hline $\begin{array}{l}\text { Power Purchase } \\
\text { Agreement ID }\end{array}$ & PPA_ID & ID & $\begin{array}{l}\text { Unique identification code assigned to each HS_PPA } \\
\text { record }\end{array}$ \\
\hline Existing Fleet Plant ID & EF_PtID & ID & $\begin{array}{l}\text { Unique identification code assigned to each HS_EF_Plant } \\
\text { record }\end{array}$ \\
\hline Year & Year & $\mathrm{N} / \mathrm{A}$ & Year of transaction \\
\hline HMR Region & HMR_Regn & $\mathrm{N} / \mathrm{A}$ & Region where plant is located \\
\hline Median Energy Price & MedianP & 2016\$/MWh & $\begin{array}{l}\text { Median energy price paid by utilities for purchased } \\
\text { electricity from hydropower plants }\end{array}$ \\
\hline $\begin{array}{l}\text { 10th Percentile Energy } \\
\text { price }\end{array}$ & 10thPrcP & 2016\$/MWh & $\begin{array}{l}\text { 10th percentile from distribution of energy prices paid by } \\
\text { utilities for purchased electricity from hydropower plants }\end{array}$ \\
\hline $\begin{array}{l}\text { 90th Percentile Energy } \\
\text { price }\end{array}$ & 90thPrcP & 2016\$/MWh & $\begin{array}{l}\text { 90th percentile from distribution of energy prices paid by } \\
\text { utilities for purchased electricity from hydropower plants }\end{array}$ \\
\hline Average & AverageP & 2016\$/MWh & $\begin{array}{l}\text { Average energy price paid by utilities for purchased } \\
\text { electricity from hydropower plants }\end{array}$ \\
\hline Number of observations & nobs & N/A & $\begin{array}{l}\text { Number of hydropower purchase transactions reported by } \\
\text { utilities to FERC Form } 1\end{array}$ \\
\hline
\end{tabular}




\section{ENVIRONMENTAL}

HSDM: HS_DG: Summary of environmental data components

\begin{tabular}{|c|c|c|c|}
\hline Data_Component & Alias & Type & Description \\
\hline HS_SiteClassAttributes & $\begin{array}{l}\text { Site Classification } \\
\text { Stream-Reach } \\
\text { Attributes }\end{array}$ & $\begin{array}{l}\text { Geospatial polyline } \\
\text { layer }\end{array}$ & $\begin{array}{l}\text { Attributes compiled from multiple layers } \\
\text { geospatially referenced to NHDPlus v2 } \\
\text { stream-reach data hosted within the Standard } \\
\text { Modular Hydropower (SMH) Explorer, a } \\
\text { geovisual analytics platform that empowers } \\
\text { user-guided energy-water-environment- } \\
\text { module data analysis and inquiries in support } \\
\text { of ORNL's SMH research project. }\end{array}$ \\
\hline HS_Mitigation & $\begin{array}{l}\text { Non-Federal } \\
\text { Hydropower } \\
\text { Mitigation Database } \\
\text { Version } 1\end{array}$ & Nonspatial table & $\begin{array}{l}\text { Mitigation requirements and associated } \\
\text { licensing information collated from FERC } \\
\text { hydropower licenses issued from } 1998 \\
\text { through } 2013 \text {. }\end{array}$ \\
\hline HS_E_StreamClass & $\begin{array}{l}\text { Eastern Stream } \\
\text { Classification }\end{array}$ & $\begin{array}{l}\text { Geospatial polyline } \\
\text { layer }\end{array}$ & $\begin{array}{l}\text { The eastern stream classification (ESC) is a } \\
\text { characterization of the biophysical settings of } \\
\text { stream environments including hydrology, } \\
\text { temperature, geomorphology, and } \\
\text { disturbance regimes. }\end{array}$ \\
\hline HS_EAFedListedFish & $\begin{array}{l}\text { Environmental } \\
\text { Attribution Federally } \\
\text { Listed Fish Species } \\
\text { by Sub-basin }\end{array}$ & $\begin{array}{l}\text { Geospatial polygon } \\
\text { layer }\end{array}$ & $\begin{array}{l}\text { Maps of fish species listed under the } \\
\text { Endangered Species Act (1973) or by the } \\
\text { International Union for the Conservation of } \\
\text { Nature (IUCN) per 8-digit HUC (HUC08) } \\
\text { were created as part of the DOE/ORNL New } \\
\text { Stream-Reach Development (NSD) Resource } \\
\text { Assessment (http://nhaap.ornl.gov/nsd). }\end{array}$ \\
\hline HS_EAFishTraits & $\begin{array}{l}\text { Environmental } \\
\text { Attribution US Fish- } \\
\text { Traits by Sub-basin }\end{array}$ & $\begin{array}{l}\text { Geospatial polygon } \\
\text { layer }\end{array}$ & $\begin{array}{l}\text { Maps of fish traits per HUC- } 8 \text { created as } \\
\text { apart of the DOE/ORNL NSD Resource } \\
\text { Assessment (http://nhaap.ornl.gov/nsd). }\end{array}$ \\
\hline HS_EAHydroClass & $\begin{array}{l}\text { Environmental } \\
\text { Attribution US } \\
\text { Hydrologic } \\
\text { Classification by Sub- } \\
\text { basin }\end{array}$ & $\begin{array}{l}\text { Geospatial polygon } \\
\text { layer }\end{array}$ & $\begin{array}{l}\text { Maps of hydrologic classes per HUC-8 were } \\
\text { created as apart of the DOE/ORNL NSD } \\
\text { Resource Assessment } \\
\text { (http://nhaap.ornl.gov/nsd). }\end{array}$ \\
\hline HS_EAWaterUse & $\begin{array}{l}\text { US Water Use } \\
\text { Distribution by Sub- } \\
\text { basin }\end{array}$ & $\begin{array}{l}\text { Geospatial polygon } \\
\text { layer }\end{array}$ & $\begin{array}{l}\text { Maps of estimated water use (2005), } \\
\text { population density, housing density, } \\
\text { precipitation, and potential } \\
\text { evapotranspiration per HUC- } 8 \text { created as } \\
\text { apart of the DOE/ORNL NSD Resource } \\
\text { Assessment (http://nhaap.ornl.gov/nsd). }\end{array}$ \\
\hline
\end{tabular}


HS_SiteClassAttributes: Attribute summary

\begin{tabular}{|c|c|c|c|}
\hline Alias & Field Name & Unit & Description \\
\hline \multirow{23}{*}{$\begin{array}{l}\text { SMH Site } \\
\text { Class ID }\end{array}$} & SiteClassID & ID & Unique database identifier \\
\hline & COMID & ID & Unique stream-reach identifier \\
\hline & HUC_6 & ID & 6-digit HUC watershed \\
\hline & gnis_name & & Stream name \\
\hline & streamorde & & Strahler stream order \\
\hline & minelevsmo & TBD & Elevation \\
\hline & slope & TBD & Slope of stream reach \\
\hline & qa_ma & $\mathrm{cfs}$ & Mean annual flow from runoff (cfs) \\
\hline & va_ma & fps & Mean annual velocity for a flowline \\
\hline & wsareasqkm & TBD & Total upstream drainage area \\
\hline & rckdepcat & $\mathrm{cm}$ & Mean depth to bedrock in catchment $(\mathrm{cm})$ \\
\hline & pctclayws & $\%$ & Percent clay content of soils in watershed \\
\hline & pctsandws & $\%$ & Percent sand content of soils in watershed \\
\hline & wq10clstrs & & Water quality clusters \\
\hline & sed10clstr & & Sediment clusters \\
\hline & gen $10 \mathrm{clstr}$ & & Generation clusters \\
\hline & npd_count & & Number of non-powered dams (NPDs) in reach \\
\hline & npd_mw & & Total potential megawatts from NPDs in reach \\
\hline & grp1 & & $\begin{array}{l}\text { Number of ocean-run sturgeon species (Acipenseriformes) } \\
\text { within the reach's HUC8 (count) }\end{array}$ \\
\hline & grp2 & & $\begin{array}{l}\text { Number of inland sturgeon/paddlefish species } \\
\text { (Acipenseriformes) within the reach's HUC8 (count) }\end{array}$ \\
\hline & grp3 & & $\begin{array}{l}\text { Number of ocean-run clupeid species within the reach's } \\
\text { HUC8 (count) }\end{array}$ \\
\hline & grp4 & & $\begin{array}{l}\text { Number of ocean-run eel/lamprey species within the reach's } \\
\text { HUC8 (count) }\end{array}$ \\
\hline & grp5 & & $\begin{array}{l}\text { Number of ocean-run salmonid species within the reach's } \\
\text { HUC8 (count) }\end{array}$ \\
\hline
\end{tabular}




\begin{tabular}{|c|c|c|}
\hline grp6 & & $\begin{array}{l}\text { Number of inland salmonid species within the reach's } \\
\text { HUC8 (count) }\end{array}$ \\
\hline grp7 & & $\begin{array}{l}\text { Number of other inland migratory species within the reach's } \\
\text { HUC8 }\end{array}$ \\
\hline fpsg10clst & & Fish passage clusters \\
\hline dist2sub & & Distance to nearest substation from reach midpoint \\
\hline state & & State that reach lies in \\
\hline supportrps & & $\begin{array}{l}\text { Percent of residents within county who support renewable } \\
\text { energy portfolio standards }\end{array}$ \\
\hline subregid & & NERC Subregion ID \\
\hline popchngsub & & $\begin{array}{l}\text { Projected population increase (in millions) by } 2050 \text { in } \\
\text { NERC subregion }\end{array}$ \\
\hline umct & & Upstream mainstem dam count \\
\hline unct & & Total upstream dam count \\
\hline dmct & & Downstream mainstem dam count \\
\hline tmct & & Total mainstem dam count \\
\hline um $2 d$ & TBD & Distance to upstream mainstem dam \\
\hline $\mathrm{dm} 2 \mathrm{~d}$ & TBD & Distance to downstream mainstem dam \\
\hline $\operatorname{tm} 2 d$ & TBD & $\begin{array}{l}\text { Total mainstem distance between upstream and/or } \\
\text { downstream mainstem dams }\end{array}$ \\
\hline udor & & $\begin{array}{l}\text { Percentage of estimated annual discharge stored in upstream } \\
\text { reservoirs }\end{array}$ \\
\hline d303_temp & & $\begin{array}{l}\text { Stream listed as impaired for temperature on US } \\
\text { Environmental Protection Agency (EPA) 303d list }\end{array}$ \\
\hline d303_sdmnt & & Stream listed as impaired for sediment on EPA 303d list \\
\hline d303_ph & & Stream listed as impaired for $\mathrm{pH}$ on EPA $303 \mathrm{~d}$ list \\
\hline d303_ntrnt & & Stream listed as impaired for nutrients on EPA 303d list \\
\hline d303_trbdt & & Stream listed as impaired for turbidity on EPA 303d list \\
\hline d303_do & & Stream listed as impaired for DO on EPA 303d list \\
\hline grndaccel & TBD & Earthquake susceptibility \\
\hline pctagcat & $\%$ & Percent agricultural land cover in catchment \\
\hline pctagws & $\%$ & Percent agricultural land cover in watershed \\
\hline
\end{tabular}




\begin{tabular}{|c|c|c|}
\hline kffactcat & & $\begin{array}{l}\text { The Kffactor-relative index of susceptibility of bare, } \\
\text { cultivated soil to particle detachment and transport by } \\
\text { rainfall in catchment }\end{array}$ \\
\hline kffactws & & $\begin{array}{l}\text { The Kffactor-relative index of susceptibility of bare, } \\
\text { cultivated soil to particle detachment and transport by } \\
\text { rainfall in watershed }\end{array}$ \\
\hline ieofcat & TBD & Mean infiltration-excess overland flow in catchment \\
\hline farmncat & TBD & Sum total of nitrogen from farm areas in catchment \\
\hline pctforwetcat & $\%$ & Percent forest and wetland land cover in watershed \\
\hline pctforwetws & $\%$ & Percent forest and wetland land cover in watershed \\
\hline pctimprv06cat & $\%$ & Percent imperviousness from 2006 in catchment \\
\hline pctimprv06ws & $\%$ & Percent imperviousness from 2006 in watershed \\
\hline popdns10cat & TBD & Population density from 2010 census in catchment \\
\hline petforripws & $\%$ & Percent riparian forest land cover in watershed \\
\hline runoffws & TBD & Mean runoff in watershed \\
\hline qa_cv & & $\begin{array}{l}\text { Coefficient of variation for flow based on monthly averages } \\
\text { and annual mean }\end{array}$ \\
\hline elevdiffws & TBD & $\begin{array}{l}\text { Difference between maximum and minimum elevation in } \\
\text { watershed }\end{array}$ \\
\hline bficat & & Base-flow index in catchment \\
\hline damundr & $\begin{array}{l}\# / 100 \\
\mathrm{~km}\end{array}$ & $\begin{array}{l}\text { Upstream network dam density per unit stream network } \\
\text { length }(\# / 100 \mathrm{~km})\end{array}$ \\
\hline damdmd & $\begin{array}{l}\# / 100 \\
\mathrm{~km}\end{array}$ & $\begin{array}{l}\text { Downstream mainstem dam density per unit downstream } \\
\text { mainstem length }(\# / 100 \mathrm{~km})\end{array}$ \\
\hline huc2prentfp & & $\begin{array}{l}\text { Percent of mitigation sites in the mitigation database within } \\
\text { the HUC } 2 \text { that had Tier } 1 \text { fish passage mitigation required }\end{array}$ \\
\hline fnd10clstrs & & Foundation clusters \\
\hline boat_ramp & & Number of developed boat ramps in HUC6 (count) \\
\hline boat_ramp_undeveloped & & Number of undeveloped boat ramps in HUC6 (count) \\
\hline fishing_coldwater & & Number of cold water fishing locations in HUC6 (count) \\
\hline fishing_saltwater & & Saltwater fishing locations in HUC6 (count) \\
\hline awhuc6km & $\mathrm{m}$ & $\begin{array}{l}\text { Length of stream }(\mathrm{m}) \text { identified as whitewater paddling runs } \\
\text { in HUC6 }\end{array}$ \\
\hline
\end{tabular}




$\begin{array}{lll}\text { nrirechuc6km } & \mathrm{m} & \begin{array}{l}\text { Length of stream (m) identified as having outstanding } \\ \text { recreational value in HUC6 }\end{array} \\ \text { popdns10huc6 } & \text { TBD } & \text { Mean population density in HUC6 } \\ \text { rec10clstrs } & \text { Recreation clusters } \\ \text { nsd_count } & \text { Number of NSD sites in reach } \\ \text { nsd_mw } & \text { Total potential megawatts from NSD sites in reach } \\ \text { genclusterdef } & \text { Short text description of generation clusters } \\ \text { wqclusterdef } & & \text { Short text description of water quality clusters } \\ \text { sedclusterdef } & \text { Short text description of sediment clusters } \\ \text { fshclusterdef } & \text { Short text description of fish passage clusters } \\ \text { fndclusterdef } & & \text { Short text description of foundation clusters } \\ \text { recclusterdef } & & \text { Short text description of recreation clusters } \\ \text { st_length(shape) } & & \text { Reach length in meters }\end{array}$

HS_Mitigation: Attribute summary

\begin{tabular}{|c|c|c|c|}
\hline Alias & Field Name & Unit & Description \\
\hline $\begin{array}{l}\text { Mitigation Database Record } \\
\text { Identifier }\end{array}$ & RecordID & Number & $\begin{array}{l}\text { Unique record identifier for each mitigation record } \\
\text { entry in MITDB }\end{array}$ \\
\hline Mitigation ID & MitigationID & String & $\begin{array}{l}\text { Unique mitigation identifier code for each } \\
\text { mitigation name }\end{array}$ \\
\hline Existing Fleet Plant ID & EF_PtID & ID & $\begin{array}{l}\text { Unique identification code assigned to each } \\
\text { HS_EF_Plant record }\end{array}$ \\
\hline Mitigation Name & Mitigation & String & Name of mitigation requirement \\
\hline $\begin{array}{l}\text { Tier Two Mitigation } \\
\text { Classification }\end{array}$ & T2_Category & String & $\begin{array}{l}\text { Second-tier classification of the mitigation } \\
\text { requirement }\end{array}$ \\
\hline Tier 2 Identifier Code & T2ID & String & Unique Tier 2 identifier code for each Tier 2 type \\
\hline $\begin{array}{l}\text { Tier One Mitigation } \\
\text { Classification }\end{array}$ & T1_Category & String & Top tier classification of the mitigation requirement \\
\hline Tier 1 Identifier Code & T1ID & String & Unique Tier 1 identifier code for each Tier 1 type \\
\hline State & State & String & Hydropower plant state location \\
\hline County & County & String & Hydropower plant county location \\
\hline
\end{tabular}




$\begin{array}{llll}\text { 8-digit Hydrologic Unit Code } & \text { HUC08 } & \text { String } & \text { Hydropower plant location by 8-digit HUC } \\ \text { 2-digit Hydrologic Unit Code } & \text { HUC02 } & \text { String } & \text { Hydropower plant location by 2-digit HUC } \\ \text { Water Body Name } & \text { Water } & \text { String } & \text { Water body associated with hydropower plant } \\ \text { FERC License Date } & \text { FcIssue } & \text { Date } & \text { Date FERC license was issued } \\ \text { FERC License Docket Number } & \text { FC_Dock } & \text { String } & \text { Identifier code for FERC license }\end{array}$

HS_E_StreamClass: Attribute summary

\begin{tabular}{|c|c|c|c|}
\hline Alias & Field Name & Unit & Description \\
\hline & FID & & Feature identifier \\
\hline & COMID & & NHDplus V1 segment identifier \\
\hline & GNIS_NAME & & Stream name \\
\hline & FTYPE & & Feature type (stream river, ArtificialPath) \\
\hline & CUMDRAINAG & & Cumulative drainage area (square kilometers) \\
\hline & Size & & $\begin{array}{l}\text { Size class (headwater, creek, small river, medium river, mainstem, large } \\
\text { river, great river) }\end{array}$ \\
\hline & Gradient & & $\begin{array}{l}\text { Gradient class (very low, low-moderate, moderate, moderate-high, high, } \\
\text { high-steep, steep, very steep) }\end{array}$ \\
\hline & Confinement & & Confinement class (confined, moderately unconfined, unconfined) \\
\hline & Substrate & & $\begin{array}{l}\text { Substrate class (sand-fine gravel, coarse gravel, small cobble, large } \\
\text { cobble, small boulder, large boulder-bedrock) }\end{array}$ \\
\hline & Hydrology & & $\begin{array}{l}\text { Hydrologic class (IF-Intermittent Flashy, LTR_-Late Timing Runoff, } \\
\text { PR1-Perennial Runoff 1, PR2- Perennial Runoff 2, SSGW-Super } \\
\text { Stable Groundwater, SHBF- Stable High Baseflow, SNM2- Snowmelt 2, } \\
\text { UI-Unpredictable Intermittent, IF2-Intermittent Flashy 2) }\end{array}$ \\
\hline & Temperature & & Temperature class (cold, cold-cool, cool, cool-warm, warm) \\
\hline & HUC & & Hydrologic region (2-digit) (regions 1-6) \\
\hline & mean_diam & & Modeled mean substrate diameter (mm) \\
\hline & TempC & & Modeled average July-August temperature in degrees Celsius \\
\hline & Slope & & Slope of the stream reach (rise/run) from NHDPlus V1 \\
\hline & Flow & & Mean annual discharge in cubic feet \\
\hline & Length_km & & Length of stream reach (kilometers) \\
\hline
\end{tabular}




\begin{tabular}{|c|c|}
\hline WaterBody & Binary indication of whether stream reach intersects reservoir or lake \\
\hline NHD_area & $\begin{array}{l}\text { Binary indication of whether stream reach intersects an NHD area } \\
\text { (typically impounded stretch of river) }\end{array}$ \\
\hline Stor 1 & Cumulative upstream dam storage (acre feet) \\
\hline DOR & $\begin{array}{l}\text { Degree of regulation: (cubic feet storage by upstream dams/annual flow } \\
\text { volume in cubic feet) } \times 100\end{array}$ \\
\hline Urban & $\begin{array}{l}\% \text { developed landcover in upstream network (NLCD } 2006 \text {, includes } \\
\text { codes } 21,22,23,24-\% \text { open space, } \% \text { low intensity, \%med intensity, } \\
\% \text { high intensity development) }\end{array}$ \\
\hline Agr & $\begin{array}{l}\% \text { agriculture landcover in upstream network (NLCD 2006, includes } \\
\text { codes } 81 \text { and } 82-\% \text { Cropland and \%Pasture) }\end{array}$ \\
\hline Forest & $\begin{array}{l}\% \text { forested land cover in upstream network (NLCD 2006, includes codes } \\
41,42 \text {, and } 43 \text { ) }\end{array}$ \\
\hline OceanFlag & $\begin{array}{l}\text { Binary indication of whether stream reach is connected (free-flowing) to } \\
\text { an estuary }\end{array}$ \\
\hline GrtLkFlag & $\begin{array}{l}\text { Binary indication of whether stream reach is connected (free-flowing) to } \\
\text { a great lake }\end{array}$ \\
\hline DCI_up & $\begin{array}{l}\text { Dendritic connectivity index (from Cote et al. 2009-Landscape } \\
\text { Ecology): Functional network/total upstream network } \times 100\end{array}$ \\
\hline NFHP & $\begin{array}{l}\text { National Fish Habitat Partnership Fish Habitat Risk Score (from } \\
\text { Esselmen et al. 2011-Ecological Indicators) }\end{array}$ \\
\hline Fish & $\begin{array}{l}\text { Binary indication of whether stream reach was previously surveyed } \\
\text { (post-1990) for fish }\end{array}$ \\
\hline Mussel & $\begin{array}{l}\text { Binary indication of whether stream reach was previously surveyed } \\
\text { (post-1990) for mussels }\end{array}$ \\
\hline Crayfish & $\begin{array}{l}\text { Binary indication of whether stream reach was previously surveyed } \\
\text { (post-1990) for crayfish }\end{array}$ \\
\hline Biota & $\begin{array}{l}\text { Binary indication of whether stream reach was previously surveyed } \\
\text { (post-1990) for any of the above biota }\end{array}$ \\
\hline Simple & Simple stream classification typologies (excludes substrate) \\
\hline Composite & Composite stream classification typologies (includes all classes) \\
\hline Dist_Reach & $\begin{array}{l}\text { Disturbance condition of each reach (not disturbed, impounded, } \\
\text { landscape alteration, fragmented, regulated) }\end{array}$ \\
\hline Hydrol_prb & $\begin{array}{l}\text { Probability of hydrology class membership based on random forest } \\
\text { model }\end{array}$ \\
\hline
\end{tabular}


HS_EAFedListedFish: Attribute summary

\begin{tabular}{|c|c|c|c|}
\hline Alias & $\begin{array}{c}\text { Field } \\
\text { Name }\end{array}$ & Unit & Description \\
\hline $\begin{array}{l}\text { Federally Listed } \\
\text { Fish ID }\end{array}$ & & ID & Unique identification code assigned to each HS_EAFedListedFish record. \\
\hline $\begin{array}{l}\text { Hydrologic } \\
\text { Region }\end{array}$ & & $\mathrm{N} / \mathrm{A}$ & Name for each 2-digit hydrologic unit \\
\hline $\begin{array}{l}\text { Hydrologic } \\
\text { Subregion }\end{array}$ & & $\mathrm{N} / \mathrm{A}$ & Name for each 4-digit hydrologic unit \\
\hline Hydrologic Basin & & $\mathrm{N} / \mathrm{A}$ & Name for each 6-digit hydrologic unit \\
\hline $\begin{array}{l}\text { Hydrologic } \\
\text { Subbasin }\end{array}$ & & $\mathrm{N} / \mathrm{A}$ & Name for each 8-digit hydrologic unit \\
\hline $\begin{array}{l}\text { 2-digit } \\
\text { Hydrologic Unit } \\
\text { Code }\end{array}$ & & $\mathrm{N} / \mathrm{A}$ & Identification Code for each 2-digit hydrologic unit \\
\hline $\begin{array}{l}\text { 4-digit } \\
\text { Hydrologic Unit } \\
\text { Code }\end{array}$ & & $\mathrm{N} / \mathrm{A}$ & Identification Code for each 4-digit hydrologic unit \\
\hline $\begin{array}{l}\text { 6-digit } \\
\text { Hydrologic Unit } \\
\text { Code }\end{array}$ & & $\mathrm{N} / \mathrm{A}$ & Identification Code for each 6-digit hydrologic unit \\
\hline $\begin{array}{l}\text { 8-digit } \\
\text { Hydrologic Unit } \\
\text { Code }\end{array}$ & & $\mathrm{N} / \mathrm{A}$ & Identification Code for each 8-digit hydrologic unit \\
\hline $\begin{array}{l}\text { Acreage per 8- } \\
\text { digit HUC }\end{array}$ & & Acre & Acreage per 8-digit hydrologic unit \\
\hline $\begin{array}{l}\text { Square miles per } \\
\text { 8-digit HUC }\end{array}$ & & $\begin{array}{l}\text { Square } \\
\text { miles }\end{array}$ & Square miles per 8-digit hydrologic unit \\
\hline $\begin{array}{l}\text { 8-digit HUC } \\
\text { State }\end{array}$ & & $\mathrm{N} / \mathrm{A}$ & Dominant representative state per 8-digit hydrologic unit \\
\hline Total fish species & & $\mathrm{N} / \mathrm{A}$ & $\begin{array}{l}\text { The total number of fish species potentially occurring within each 8-HUC } \\
\text { watershed. Fish species distributions were available from NatureServe } \\
\text { (http://www.natureserve.org/getData/fishMaps.jsp) within 8-digit HUCs from } \\
\text { the HUC250K version of watershed boundaries. } \\
\text { (http://water.usgs.gov/GIS/huc.html). Fish species were resummarized into 8- } \\
\text { digit HUC boundaries within the Watershed Boundary Dataset. }\end{array}$ \\
\hline $\begin{array}{l}\text { Number of } \\
\text { endangered fish } \\
\text { species under } \\
\text { ESA }\end{array}$ & & $\mathrm{N} / \mathrm{A}$ & $\begin{array}{l}\text { The number of fish species listed as "Endangered" under the Endangered } \\
\text { Species Act (ESA) (1973). Fish species distributions were available from } \\
\text { NatureServe (http://www.natureserve.org/getData/fishMaps.jsp) within 8-digit } \\
\text { HUCs from the HUC250K version of watershed boundaries. }\end{array}$ \\
\hline
\end{tabular}


Number of threatened fish species under ESA

Number of candidate fish species under ESA

Number of fish species of concern under ESA

Number of proposed endangered fish species under ESA

Number of proposed threatened fish species under ESA

Number of fish species with populations as
(http://water.usgs.gov/GIS/huc.html). Fish species were resummarized into 8digit HUC boundaries within the Watershed Boundary Dataset. Information on listed status was obtained through Nature Serve Explorer (http://www.natureserve.org/explorer/).

N/A The number of fish species listed as "Threatened" under the ESA (1973). Fish species distributions were available from NatureServe (http://www.natureserve.org/getData/fishMaps.jsp) within 8-digit HUCs from the HUC250K version of watershed boundaries.

(http://water.usgs.gov/GIS/huc.html). Fish species were resummarized into 8digit HUC boundaries within the Watershed Boundary Dataset. Information on listed status was obtained through Nature Serve Explorer (http://www.natureserve.org/explorer/).

N/A The number of fish species listed as "Candidate" species under the ESA (1973). Fish species distributions were available from NatureServe (http://www.natureserve.org/getData/fishMaps.jsp) within 8-digit HUCs from the HUC250K version of watershed boundaries. (http://water.usgs.gov/GIS/huc.html). Fish species were resummarized into 8digit HUC boundaries within the Watershed Boundary Dataset. Information on listed status was obtained through Nature Serve Explorer (http://www.natureserve.org/explorer/).

N/A The number of fish species listed as "Species of Concern" under the ESA (1973). Fish species distributions were available from NatureServe (http://www.natureserve.org/getData/fishMaps.jsp) within 8-digit HUCs from the HUC250K version of watershed boundaries.

(http://water.usgs.gov/GIS/huc.html). Fish species were resummarized into 8digit HUC boundaries within the Watershed Boundary Dataset. Information on listed status was obtained through Nature Serve Explorer (http://www.natureserve.org/explorer/).

N/A The number of fish species proposed as "Endangered" under the ESA (1973). Fish species distributions were available from NatureServe (http://www.natureserve.org/getData/fishMaps.jsp) within 8-digit HUCs from the HUC250K version of watershed boundaries.

(http://water.usgs.gov/GIS/huc.html). Fish species were resummarized into 8digit HUC boundaries within the Watershed Boundary Dataset. Information on listed status was obtained through Nature Serve Explorer (http://www.natureserve.org/explorer/).

N/A The number of fish species proposed as "Threatened" under the ESA (1973). Fish species distributions were available from NatureServe (http://www.natureserve.org/getData/fishMaps.jsp) within 8-digit HUCs from the HUC250K version of watershed boundaries.

(http://water.usgs.gov/GIS/huc.html). Fish species were resummarized into 8digit HUC boundaries within the Watershed Boundary Dataset. Information on listed status was obtained through Nature Serve Explorer (http://www.natureserve.org/explorer/).

N/A The number of fish species with a population (not entire species) listed as "Endangered" under the ESA (1973). Fish species distributions were available from NatureServe (http://www.natureserve.org/getData/fishMaps.jsp) within 
endangered under ESA

Number of fish species with populations as threatened under ESA

Number of fish species with populations as candidates under ESA

Number of endangered or threatened fish species under ESA

Number of fish species falling under ESA designation

Number of IUCN critically endangered fish species 8-digit HUCs from the HUC250K version of watershed boundaries. (http://water.usgs.gov/GIS/huc.html). Fish species were resummarized into 8digit HUC boundaries within the Watershed Boundary Dataset. Information on listed status was obtained through Nature Serve Explorer (http://www.natureserve.org/explorer/).

N/A The number of fish species with a population (not entire species) listed as "Threatened" under the ESA (1973). Fish species distributions were available from NatureServe (http://www.natureserve.org/getData/fishMaps.jsp) within 8-digit HUCs from the HUC250K version of watershed boundaries. (http://water.usgs.gov/GIS/huc.html). Fish species were resummarized into 8digit HUC boundaries within the Watershed Boundary Dataset. Information on listed status was obtained through Nature Serve Explorer (http://www.natureserve.org/explorer/).

N/A The number of fish species with a population (not entire species) proposed as a "Candidate" species under the ESA (1973). Fish species distributions were available from NatureServe (http://www.natureserve.org/getData/fishMaps.jsp) within 8-digit HUCs from the HUC250K version of watershed boundaries. (http://water.usgs.gov/GIS/huc.html). Fish species were resummarized into 8digit HUC boundaries within the Watershed Boundary Dataset. Information on listed status was obtained through Nature Serve Explorer (http://www.natureserve.org/explorer/).

N/A The number of fish species listed as "Endangered" or "Threatened" under the ESA (1973). Fish species distributions were available from NatureServe (http://www.natureserve.org/getData/fishMaps.jsp) within 8-digit HUCs from the HUC250K version of watershed boundaries.

(http://water.usgs.gov/GIS/huc.html). Fish species were resummarized into 8digit HUC boundaries within the Watershed Boundary Dataset. Information on listed status was obtained through Nature Serve Explorer (http://www.natureserve.org/explorer/).

N/A The total number of fish species (or their populations) recognized as listed, proposed, or candidate under the ESA (1973). Fish species distributions were available from NatureServe

(http://www.natureserve.org/getData/fishMaps.jsp) within 8-digit HUCs from the HUC250K version of watershed boundaries.

(http://water.usgs.gov/GIS/huc.html). Fish species were resummarized into 8digit HUC boundaries within the Watershed Boundary Dataset. Information on listed status was obtained through Nature Serve Explorer (http://www.natureserve.org/explorer/).

N/A The number of fish species ranked as "Critically Endangered" under the criteria developed by IUCN. Fish species distributions were available from NatureServe (http:/www.natureserve.org/getData/fishMaps.jsp) within 8-digit HUCs from the HUC250K version of watershed boundaries. (http://water.usgs.gov/GIS/huc.html). Fish species were resummarized into 8digit HUC boundaries within the Watershed Boundary Dataset. Information on IUCN criteria was obtained through Nature Serve Explorer (http://www.natureserve.org/explorer/). Descriptions of IUCN criteria are available from IUCN (International Union for the Conservation of Nature 
Number of IUCN endangered fish species

Number of IUCN vulnerable fish species

Number of IUCN near threatened fish species

Number of fish species under IUCN ranking
2001), IUCN Red List categories and criteria: Version 3.1, IUCN Species Survival Commission, IUCN, Gland, Switzerland, and Cambridge, UK.

N/A The number of fish species ranked as "Endangered" under the criteria developed by the IUCN. Fish species distributions were available from NatureServe (http://www.natureserve.org/getData/fishMaps.jsp) within 8-digit HUCs from the HUC250K version of watershed boundaries. (http://water.usgs.gov/GIS/huc.html). Fish species were resummarized into 8digit HUC boundaries within the Watershed Boundary Dataset. Information on IUCN criteria was obtained through Nature Serve Explorer (http://www.natureserve.org/explorer/). Descriptions of IUCN criteria are available from IUCN (International Union for the Conservation of Nature 2001), IUCN Red List categories and criteria: Version 3.1, IUCN Species Survival Commission, IUCN, Gland, Switzerland, and Cambridge, UK.

N/A The number of fish species ranked as "Vulnerable" under the criteria developed by the IUCN. Fish species distributions were available from NatureServe (http://www.natureserve.org/getData/fishMaps.jsp) within 8-digit HUCs from the HUC250K version of watershed boundaries. (http://water.usgs.gov/GIS/huc.html). Fish species were resummarized into 8digit HUC boundaries within the Watershed Boundary Dataset. Information on IUCN criteria was obtained through Nature Serve Explorer (http://www.natureserve.org/explorer/). Descriptions of IUCN criteria are available from IUCN (International Union for the Conservation of Nature 2001), IUCN Red List categories and criteria: Version 3.1, IUCN Species Survival Commission, IUCN, Gland, Switzerland, and Cambridge, UK.

N/A The number of fish species ranked as "Near Threatened" under the criteria developed by the IUCN. Fish species distributions were available from NatureServe (http://www.natureserve.org/getData/fishMaps.jsp) within 8-digit HUCs from the HUC250K version of watershed boundaries. (http://water.usgs.gov/GIS/huc.html). Fish species were resummarized into 8digit HUC boundaries within the Watershed Boundary Dataset. Information on IUCN criteria was obtained through Nature Serve Explorer (http://www.natureserve.org/explorer/). Descriptions of IUCN criteria are available from IUCN (International Union for the Conservation of Nature 2001), IUCN Red List categories and criteria: Version 3.1, IUCN Species Survival Commission, IUCN, Gland, Switzerland, and Cambridge, UK.

N/A The number of fish species ranked as "Critically endangered," "Endangered," "Vulnerable," or "Near threatened" under the criteria developed by the IUCN. Fish species distributions were available from NatureServe (http://www.natureserve.org/getData/fishMaps.jsp) within 8-digit HUCs from the HUC250K version of watershed boundaries.

(http://water.usgs.gov/GIS/huc.html). Fish species were resummarized into 8digit HUC boundaries within the Watershed Boundary Dataset. Information on IUCN criteria was obtained through Nature Serve Explorer (http://www.natureserve.org/explorer/). Descriptions of IUCN criteria are available from IUCN (International Union for the Conservation of Nature 2001), IUCN Red List categories and criteria: Version 3.1, IUCN Species Survival Commission, IUCN, Gland, Switzerland, and Cambridge, UK. 
All fish species of concern under ESA and IUCN
N/A The number of fish species falling under the criteria of the ESA (1973) or ranking of the IUCN. Fish species distributions were available from NatureServe (http://www.natureserve.org/getData/fishMaps.jsp) within 8-digit HUCs from the HUC250K version of watershed boundaries. (http://water.usgs.gov/GIS/huc.html). Fish species were resummarized into 8digit HUC boundaries within the Watershed Boundary Dataset. Information on ESA or IUCN criteria was obtained through Nature Serve Explorer (http://www.natureserve.org/explorer/). Descriptions of IUCN criteria are available from IUCN (International Union for the Conservation of Nature 2001), IUCN Red List categories and criteria: Version 3.1, IUCN Species Survival Commission, IUCN, Gland, Switzerland, and Cambridge, UK.

\section{HS_EAFishTraits: Attribute summary}

\begin{tabular}{|c|c|c|c|}
\hline Alias & $\begin{array}{l}\text { Field } \\
\text { Name }\end{array}$ & Unit & Description \\
\hline Fish Traits ID & & ID & $\begin{array}{l}\text { Unique identification code assigned to each HS_EAFishTraits } \\
\text { record }\end{array}$ \\
\hline Hydrologic Region & & N/A & Name for each 2-digit hydrologic unit \\
\hline Hydrologic Subregion & & N/A & Name for each 4-digit hydrologic unit \\
\hline Hydrologic Basin & & N/A & Name for each 6-digit hydrologic unit \\
\hline Hydrologic Subbasin & & N/A & Name for each 8-digit hydrologic unit \\
\hline $\begin{array}{l}\text { 2-digit Hydrologic Unit } \\
\text { Code }\end{array}$ & & $\mathrm{N} / \mathrm{A}$ & Identification Code for each 2-digit hydrologic unit \\
\hline $\begin{array}{l}\text { 4-digit Hydrologic Unit } \\
\text { Code }\end{array}$ & & N/A & Identification Code for each 4-digit hydrologic unit \\
\hline $\begin{array}{l}\text { 6-digit Hydrologic Unit } \\
\text { Code }\end{array}$ & & N/A & Identification Code for each 6-digit hydrologic unit \\
\hline $\begin{array}{l}\text { 8-digit Hydrologic Unit } \\
\text { Code }\end{array}$ & & $\mathrm{N} / \mathrm{A}$ & Identification Code for each 8-digit hydrologic unit \\
\hline Acreage per 8-digit HUC & & Acre & Acreage per 8-digit hydrologic unit \\
\hline $\begin{array}{l}\text { Square miles per 8-digit } \\
\text { HUC }\end{array}$ & & $\begin{array}{l}\text { Square } \\
\text { miles }\end{array}$ & Square miles per 8-digit hydrologic unit \\
\hline 8-digit HUC State & & N/A & Dominant representative state per 8-digit hydrologic unit \\
\hline Total fish species & & $\mathrm{N} / \mathrm{A}$ & $\begin{array}{l}\text { The total number of fish species potentially occurring within each 8- } \\
\text { HUC Watershed. Fish species distributions were available from } \\
\text { NatureServe (http://www.natureserve.org/getData/fishMaps.jsp) } \\
\text { within 8-digit HUCs from the HUC250K version of watershed } \\
\text { boundaries. (http://water.usgs.gov/GIS/huc.html). Fish species were }\end{array}$ \\
\hline
\end{tabular}


Native fish species

Serial spawning fish species

Lotic specialist/Serial spawning fish species

Fish species with temporally restricted spawning seasons resummarized into 8-digit HUC boundaries within the Watershed Boundary Dataset.

N/A The number of fish species native to North America potentially occurring within each 8-HUC Watershed. Fish species distributions were available from NatureServe (http://www.natureserve.org/getData/fishMaps.jsp) within 8-digit HUCs from the HUC250K version of watershed boundaries. (http://water.usgs.gov/GIS/huc.html). Fish species were resummarized into 8-digit HUC boundaries within the Watershed Boundary Dataset. Information on native species status for fishes was obtained through the Fish Traits Database (http://fishwild.vt.edu/fishtraits/). See also Frimpong, E. A., and P. L. Angermeier (2009), "Fish Traits: A database of Ecological and Life-history traits of freshwater fishes of the United States," Fisheries 34: 487-495.

N/A The number of fish species documented as serial spawners within each 8-HUC Watershed. Serial spawning fish species (i.e., batch spawners) spawn multiple times per spawning season, as evidence of multiple clutches or separate distinct batches of eggs at various stages of maturation. Fish species distributions were available from NatureServe (http://www.natureserve.org/getData/fishMaps.jsp) within 8-digit HUCs from the HUC250K version of watershed boundaries. (http://water.usgs.gov/GIS/huc.html). Fish species were resummarized into 8-digit HUC boundaries within the Watershed Boundary Dataset. Fish trait information was obtained through the Fish Traits Database (http://fishwild.vt.edu/fishtraits/). See also Frimpong, E. A., and P. L. Angermeier (2009), "Fish Traits: A database of Ecological and Life-history traits of freshwater fishes of the United States," Fisheries 34: 487-495.

N/A The number of fish species documented as serial spawners and preference for lotic habitats within each 8-HUC Watershed. Serial spawning fish species (i.e. batch spawners) spawn multiple times per spawning season, as evidence of multiple clutches or separate distinct batches of eggs at various stages of maturation. Lotic specialist fish prefer habitats with moving water. Fish species distributions were available from NatureServe

(http://www.natureserve.org/getData/fishMaps.jsp) within 8-digit HUCs from the HUC250K version of watershed boundaries. (http://water.usgs.gov/GIS/huc.html). Fish species were resummarized into 8-digit HUC boundaries within the Watershed Boundary Dataset. Fish trait information was obtained through the Fish Traits Database (http://fishwild.vt.edu/fishtraits/). See also Frimpong, E. A., and P. L. Angermeier (2009), "Fish Traits: A database of Ecological and Life-history traits of freshwater fishes of the United States," Fisheries 34: 487-495.

N/A The number of fish species with temporally restricted spawning seasons potentially occurring within each 8-HUC Watershed. Species with temporally restricted spawning seasons (i.e., high spawning seasonality) were identified by species falling within the 
Lotic specialist/temporally restricted spawning fish species

Habitat specialist fish species

Lotic specialist/habitat specialist fish species lowest tenth percentile spawning season duration (number of months) for all species. Fish species distributions were available from NatureServe

(http://www.natureserve.org/getData/fishMaps.jsp) within 8-digit HUCs from the HUC250K version of watershed boundaries. (http://water.usgs.gov/GIS/huc.html). Fish species were resummarized into 8-digit HUC boundaries within the Watershed Boundary Dataset. Fish trait information was obtained through the Fish Traits Database (http://fishwild.vt.edu/fishtraits/). See also Frimpong, E. A., and P. L. Angermeier (2009), "Fish Traits: A database of Ecological and Life-history traits of freshwater fishes of the United States," Fisheries 34: 487-495.

N/A The number of fish species with temporally restricted spawning seasons and preference for lotic habitats potentially occurring within each 8-HUC Watershed. Species with temporally restricted spawning seasons (i.e., high spawning seasonality) were identified by species falling within the lowest tenth percentile spawning season duration (number of months) for all species. Lotic specialist fish prefer habitats with moving water. Fish species distributions were available from NatureServe (http://www.natureserve.org/getData/fishMaps.jsp) within 8-digit HUCs from the HUC250K version of watershed boundaries. (http://water.usgs.gov/GIS/huc.html). Fish species were resummarized into 8-digit HUC boundaries within the Watershed Boundary Dataset. Fish trait information was obtained through the Fish Traits Database (http://fishwild.vt.edu/fishtraits/). See also Frimpong, E. A., and P. L. Angermeier (2009), "Fish Traits: A database of Ecological and Life-history traits of freshwater fishes of the United States," Fisheries 34: 487-495.

N/A The number of fish species considered habitat specialists potentially occurring within each 8-HUC Watershed. Habitat specialist scores were calculated by summing the number of habitats (substrates/waterbody types) and diet diversity characteristic of each species. Species with lower values were presumed to have more specific habitat needs. Habitat specialists were identified as those species having habitat specialist scores within the lowest tenth percentile of all species. Fish species distributions were available from NatureServe (http://www.natureserve.org/getData/fishMaps.jsp) within 8-digit HUCs from the HUC250K version of watershed boundaries. (http://water.usgs.gov/GIS/huc.html). Fish species were resummarized into 8-digit HUC boundaries within the Watershed Boundary Dataset. Fish trait information was obtained through the Fish Traits Database (http://fishwild.vt.edu/fishtraits/). See also Frimpong, E. A., and P. L. Angermeier (2009), "Fish Traits: A database of Ecological and Life-history traits of freshwater fishes of the United States," Fisheries 34: 487-495. 
Geographically limited fish species

Potadromous or

anadromous fish species

Lotic fish species
N/A The number of fish species with geographically limited ranges potentially occurring within each 8-HUC Watershed. Species with small ranges (in square kilometers) were identified as those with ranges falling within the lowest tenth percentile of all species. Fish species distributions were available from NatureServe (http://www.natureserve.org/getData/fishMaps.jsp) within 8-digit HUCs from the HUC250K version of watershed boundaries. (http://water.usgs.gov/GIS/huc.html). Fish species were resummarized into 8-digit HUC boundaries within the Watershed Boundary Dataset. Fish trait information was obtained through the Fish Traits Database (http://fishwild.vt.edu/fishtraits/). See also Frimpong, E. A., and P. L. Angermeier (2009), "Fish Traits: A database of Ecological and Life-history traits of freshwater fishes of the United States," Fisheries 34: 487-495.

N/A The number of potadromous or anadromous fish species potentially occurring within each 8-HUC Watershed. Potadromous or anadromous were identified as exhibiting significant movement related to spawning between marine and freshwater or within freshwater. Fish species distributions were available from NatureServe (http://www.natureserve.org/getData/fishMaps.jsp) within 8-digit HUCs from the HUC250K version of watershed boundaries. (http://water.usgs.gov/GIS/huc.html). Fish species were resummarized into 8-digit HUC boundaries within the Watershed Boundary Dataset. Fish trait information was obtained through the Fish Traits Database (http://fishwild.vt.edu/fishtraits/). See also Frimpong, E. A., and P. L. Angermeier (2009), "Fish Traits: A database of Ecological and Life-history traits of freshwater fishes of the United States," Fisheries 34: 487-495.

N/A The number of fish species preferring lotic habitats potentially occurring within each 8-HUC Watershed. Lotic specialist fish prefer habitats with moving water. Fish species distributions were available from NatureServe (http://www.natureserve.org/getData/fishMaps.jsp) within 8-digit HUCs from the HUC250K version of watershed boundaries. (http://water.usgs.gov/GIS/huc.html). Fish species were resummarized into 8-digit HUC boundaries within the Watershed Boundary Dataset. Fish trait information was obtained through the Fish Traits Database (http://fishwild.vt.edu/fishtraits/). See also Frimpong, E. A., and P. L. Angermeier (2009), "Fish Traits: A database of Ecological and Life-history traits of freshwater fishes of the United States," Fisheries 34: 487-495. 
N/A The number of fish species preferring lentic habitats potentially occurring within each 8-HUC Watershed. Lentic fish species prefer habitats without moving water. Fish species distributions were available from NatureServe

(http://www.natureserve.org/getData/fishMaps.jsp) within 8-digit HUCs from the HUC250K version of watershed boundaries. (http://water.usgs.gov/GIS/huc.html). Fish species were resummarized into 8-digit HUC boundaries within the Watershed Boundary Dataset. Fish trait information was obtained through the Fish Traits database (http://fishwild.vt.edu/fishtraits/). See also Frimpong, E. A., and P. L. Angermeier (2009), "Fish Traits: A database of Ecological and Life-history traits of freshwater fishes of the United States," Fisheries 34: 487-495.

HS_EAHydroClass: Attribute Summary

\begin{tabular}{|c|c|c|c|}
\hline Alias & $\begin{array}{l}\text { Field } \\
\text { Name }\end{array}$ & Unit & Description \\
\hline $\begin{array}{l}\text { Hydrologic } \\
\text { Classification ID }\end{array}$ & & ID & Unique identification code assigned to each HS_EAHydroClass record \\
\hline Hydrologic Region & & $\mathrm{N} / \mathrm{A}$ & Name for each 2-digit hydrologic unit \\
\hline $\begin{array}{l}\text { Hydrologic } \\
\text { Subregion }\end{array}$ & & N/A & Name for each 4-digit hydrologic unit \\
\hline Hydrologic Basin & & N/A & Name for each 6-digit hydrologic unit \\
\hline $\begin{array}{l}\text { Hydrologic } \\
\text { Subbasin }\end{array}$ & & $\mathrm{N} / \mathrm{A}$ & Name for each 8-digit hydrologic unit \\
\hline $\begin{array}{l}\text { 2-digit Hydrologic } \\
\text { Unit Code }\end{array}$ & & $\mathrm{N} / \mathrm{A}$ & Identification Code for each 2-digit hydrologic unit \\
\hline $\begin{array}{l}\text { 4-digit Hydrologic } \\
\text { Unit Code }\end{array}$ & & $\mathrm{N} / \mathrm{A}$ & Identification Code for each 4-digit hydrologic unit \\
\hline $\begin{array}{l}\text { 6-digit Hydrologic } \\
\text { Unit Code }\end{array}$ & & $\mathrm{N} / \mathrm{A}$ & Identification Code for each 6-digit hydrologic unit \\
\hline $\begin{array}{l}\text { 8-digit Hydrologic } \\
\text { Unit Code }\end{array}$ & & $\mathrm{N} / \mathrm{A}$ & Identification Code for each 8-digit hydrologic unit \\
\hline $\begin{array}{l}\text { Acreage per 8-digit } \\
\text { HUC }\end{array}$ & & Acre & Acreage per 8-digit hydrologic unit \\
\hline $\begin{array}{l}\text { Square miles per 8- } \\
\text { digit HUC }\end{array}$ & & $\begin{array}{l}\text { Square } \\
\text { miles }\end{array}$ & Square miles per 8-digit hydrologic unit \\
\hline 8-digit HUC State & & $\mathrm{N} / \mathrm{A}$ & Dominant representative state per 8-digit hydrologic unit \\
\hline
\end{tabular}


Dominant

Hydrologic Class

Code

Secondary

Hydrologic Class

Code

Dominant

Hydrologic Class

Name

Secondary

Hydrologic Class

Name
N/A The dominant hydrologic class code represented within each 8-digit hydrologic unit. Hydrologic classes were created by McManamay et al. (2013) using discharge information from 2,618 USGS streamflow gages (USGS National Water Information System: http://waterdata.usgs.gov/usa/nwis/si). Hydrologic classes are groups of streams that share similar hydrologic properties, i.e., similarity in streamflow characteristics, and may respond similarly to impoundment. Theisen polygons were created for each USGS, and each dominant hydrologic class was assigned to 8-digit hydrologic unit. For descriptions of hydrologic classes, see metadata description. For more information see McManamay, R. A., M. S. Bevelhimer, and S. Kao, 2013, "A New US Hydrologic Classification: A Tool to Stratify Analyses in Ecohydrology," Ecohydrology (in review).

N/A The secondary hydrologic class code represented within each 8-digit hydrologic unit. Hydrologic classes were created by McManamay et al. (2013) using discharge information from 2,618 USGS streamflow gages (USGS National Water Information System:

http://waterdata.usgs.gov/usa/nwis/si). Hydrologic classes are groups of streams that share similar hydrologic properties, i.e. similarity in streamflow characteristics, and may respond similarly to impoundment. Theisen polygons were created for each USGS, and each dominant hydrologic class was assigned to 8-digit hydrologic units. For descriptions of hydrologic classes, see metadata description. For more information see McManamay, R. A., M. S. Bevelhimer, and S. Kao, 2013, "A New US Hydrologic Classification: A Tool to Stratify Analyses in Ecohydrology," Ecohydrology (in review).

N/A The dominant hydrologic class name represented within each 8-digit hydrologic unit. Hydrologic classes were created by McManamay et al. (2013) using discharge information from 2,618 USGS streamflow gages (USGS National Water Information System: http://waterdata.usgs.gov/usa/nwis/si). Hydrologic classes are groups of streams that share similar hydrologic properties, i.e. similarity in streamflow characteristics, and may respond similarly to impoundment. Theisen polygons were created for each USGS, and each dominant hydrologic class was assigned to 8-digit hydrologic units. For descriptions of hydrologic classes, see metadata description. For more information see McManamay, R. A., M. S. Bevelhimer, and S. Kao, 2013, "A New US Hydrologic Classification: A Tool to Stratify Analyses in Ecohydrology," Ecohydrology (in review).

N/A The dominant hydrologic class name represented within each 8-digit hydrologic unit. Hydrologic classes were created by McManamay et al. (2013) using discharge information from 2,618 USGS streamflow gages (USGS National Water Information System:

http://waterdata.usgs.gov/usa/nwis/si). Hydrologic classes are groups of streams that share similar hydrologic properties, i.e. similarity in streamflow characteristics, and may respond similarly to impoundment. Theisen polygons were created for each USGS, and each dominant hydrologic class was assigned to 8-digit hydrologic units. For descriptions of hydrologic classes, see metadata description. For more information see 
Number of

hydrologic classes

per HUC
McManamay, R. A., M. S. Bevelhimer, and S. Kao, 2013, "A New US Hydrologic Classification: A Tool to Stratify Analyses in Ecohydrology," Ecohydrology (in review).

N/A The total number of hydrologic classes represented within each 8-digit hydrologic unit. Hydrologic classes were created by McManamay et al. (2013) using discharge information from 2,618 USGS streamflow gages (USGS National Water Information System:

http://waterdata.usgs.gov/usa/nwis/si). Hydrologic classes are groups of streams that share similar hydrologic properties, i.e. similarity in streamflow characteristics, and may respond similarly to impoundment. Theisen polygons were created for each USGS, and each dominant hydrologic class was assigned to 8-digit hydrologic units. For descriptions of hydrologic classes, see metadata description. For more information see McManamay, R. A., M. S. Bevelhimer, and S. Kao, 2013, "A New US Hydrologic Classification: A Tool to Stratify Analyses in Ecohydrology," Ecohydrology (in review).

HS_EAWaterUse: Attribute summary

\begin{tabular}{|c|c|c|c|}
\hline Alias & Field Name & Unit & Description \\
\hline Water Use ID & & ID & $\begin{array}{l}\text { Unique identification code assigned to each } \\
\text { HS_EAWaterUse record }\end{array}$ \\
\hline Hydrologic Region & & $\mathrm{N} / \mathrm{A}$ & Name for each 2-digit hydrologic unit \\
\hline Hydrologic Subregion & & $\mathrm{N} / \mathrm{A}$ & Name for each 4-digit hydrologic unit \\
\hline Hydrologic Basin & & $\mathrm{N} / \mathrm{A}$ & Name for each 6-digit hydrologic unit \\
\hline Hydrologic Subbasin & & $\mathrm{N} / \mathrm{A}$ & Name for each 8-digit hydrologic unit \\
\hline $\begin{array}{l}\text { 2-digit Hydrologic } \\
\text { Unit Code }\end{array}$ & & $\mathrm{N} / \mathrm{A}$ & Identification code for each 2-digit hydrologic unit \\
\hline $\begin{array}{l}\text { 4-digit Hydrologic } \\
\text { Unit Code }\end{array}$ & & $\mathrm{N} / \mathrm{A}$ & Identification code for each 4-digit hydrologic unit \\
\hline $\begin{array}{l}\text { 6-digit Hydrologic } \\
\text { Unit Code }\end{array}$ & & $\mathrm{N} / \mathrm{A}$ & Identification code for each 6-digit hydrologic unit \\
\hline $\begin{array}{l}\text { 8-digit Hydrologic } \\
\text { Unit Code }\end{array}$ & & $\mathrm{N} / \mathrm{A}$ & Identification code for each 8-digit hydrologic unit \\
\hline $\begin{array}{l}\text { Acreage per 8-digit } \\
\text { HUC }\end{array}$ & & Acre & Acreage per 8-digit hydrologic unit \\
\hline $\begin{array}{l}\text { Square miles per 8- } \\
\text { digit HUC }\end{array}$ & & Square miles & Square miles per 8-digit hydrologic unit \\
\hline 8-digit HUC State & & $\mathrm{N} / \mathrm{A}$ & Dominant representative state per 8-digit hydrologic unit \\
\hline
\end{tabular}


Population density

House density

Precipitation

Potential

Evapotranspiration

Aquaculture water use

Domestic water use

Individuals $\mathrm{km}^{2}$

Houses $\mathrm{km}^{2}$

Inches

Inches

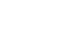


Total ground water use

Industrial water use

Irrigation water use

Livestock water use and population density were obtained through the US Census Bureau (http://www.census.gov/geo/mapsdata/data/tiger.html). Water use was summarized by 8 -digit hydrologic unit codes using area-weighted averages of county-level data.

L/day/1 km2 Average total ground water use (liters day-1 km-2) per 8digit hydrologic unit. Water use estimates (2005) per county were obtained from Kenny, J. F., Barber, N. L., Hutson, S. S., Linsey, K. S., Lovelace, J. K., and Maupin, M. A., 2009, "Estimated use of water in the United States in 2005," USGS Circular 1344, 52 p. (http://pubs.usgs.gov/circ/1344/). County-level shape files and population density were obtained through the US Census Bureau (http://www.census.gov/geo/maps-data/data/tiger.html). Water use was summarized by 8-digit hydrologic unit codes using area-weighted averages of county-level data.

L/day/1 km2 Average industrial surface water use (liters day-1 km-2) per 8-digit hydrologic unit. Water use estimates (2005) per county were obtained from Kenny, J. F., Barber, N. L., Hutson, S. S., Linsey, K. S., Lovelace, J. K., and Maupin, M. A., 2009, "Estimated use of water in the United States in 2005," USGS Circular 1344, 52 p.

(http://pubs.usgs.gov/circ/1344/). County-level shape files and population density were obtained through the US Census Bureau (http://www.census.gov/geo/mapsdata/data/tiger.html). Water use was summarized by 8 -digit hydrologic unit codes using area-weighted averages of county-level data.

L/day/1 km2 Average irrigation surface water use (liters day-1 km-2) per 8-digit hydrologic unit. Water use estimates (2005) per county were obtained from Kenny, J. F., Barber, N. L., Hutson, S. S., Linsey, K. S., Lovelace, J. K., and Maupin, M. A., 2009, "Estimated use of water in the United States in 2005," USGS Circular 1344, 52 p.

(http://pubs.usgs.gov/circ/1344/). County-level shape files and population density were obtained through the US Census Bureau (http://www.census.gov/geo/mapsdata/data/tiger.html). Water use was summarized by 8 -digit hydrologic unit codes using area-weighted averages of county-level data.

L/day/1 km2 Average livestock surface water use (liters day-1 km-2) per 8-digit hydrologic unit. Water use estimates (2005) per county were obtained from Kenny, J. F., Barber, N. L., Hutson, S. S., Linsey, K. S., Lovelace, J. K., and Maupin, M. A., 2009, "Estimated use of water in the United States in 2005," USGS Circular 1344, 52 p.

(http://pubs.usgs.gov/circ/1344/). County-level shape files and population density were obtained through the US Census Bureau (http://www.census.gov/geo/maps- 
Mining water use

Public water use

Total surface water use

Thermoelectric water use data/data/tiger.html). Water use was summarized by 8 -digit hydrologic unit codes using area-weighted averages of county-level data.

L/day/1 km2 Average mining operations surface water use (liters day-1 $\mathrm{km}$-2) per 8-digit hydrologic unit. Water use estimates (2005) per county were obtained from Kenny, J. F., Barber, N. L., Hutson, S. S., Linsey, K. S., Lovelace, J. K., and Maupin, M. A., 2009, "Estimated use of water in the United States in 2005," USGS Circular 1344, 52 p.

(http://pubs.usgs.gov/circ/1344/). County-level shape files and population density were obtained through the US Census Bureau (http://www.census.gov/geo/mapsdata/data/tiger.html). Water use was summarized by 8 -digit hydrologic unit codes using area-weighted averages of county-level data.

L/day/1 km2 Average public surface water use (liters day-1 km-2) per 8digit hydrologic unit. Water use estimates (2005) per county were obtained from Kenny, J. F., Barber, N. L., Hutson, S. S., Linsey, K. S., Lovelace, J. K., and Maupin, M. A., 2009, "Estimated use of water in the United States in 2005," USGS Circular 1344, 52 p. (http://pubs.usgs.gov/circ/1344/). County-level shape files and population density were obtained through the US Census Bureau (http://www.census.gov/geo/maps-data/data/tiger.html). Water use was summarized by 8-digit hydrologic unit codes using area-weighted averages of county-level data.

L/day/1 km2 Average total surface water use (liters day-1 km-2) per 8digit hydrologic unit. Water use estimates (2005) per county were obtained from Kenny, J. F., Barber, N. L., Hutson, S. S., Linsey, K. S., Lovelace, J. K., and Maupin, M. A., 2009, "Estimated use of water in the United States in 2005," USGS Circular 1344, 52 p. (http://pubs.usgs.gov/circ/1344/).

County-level shape files and population density were obtained through the US Census Bureau (http://www.census.gov/geo/maps-data/data/tiger.html). Water use was summarized by 8-digit hydrologic unit codes using area-weighted averages of county-level data.

L/day/1 km2 Average thermoelectric surface water use (liters day-1 km-2) per 8-digit hydrologic unit. Water use estimates (2005) per county were obtained from Kenny, J. F., Barber, N. L., Hutson, S. S., Linsey, K. S., Lovelace, J. K., and Maupin, M. A., 2009, "Estimated use of water in the United States in 2005," USGS Circular 1344, 52 p.

(http://pubs.usgs.gov/circ/1344/). County-level shape files and population density were obtained through the US Census Bureau (http://www.census.gov/geo/mapsdata/data/tiger.html). Water use was summarized by 8 -digit 
hydrologic unit codes using area-weighted averages of county-level data.

Total freshwater use

L/day/1 km2 Average total freshwater (surface and groundwater) use (liters day-1 km-2) per 8-digit hydrologic unit. Water use estimates (2005) per county were obtained from Kenny, J. F., Barber, N. L., Hutson, S. S., Linsey, K. S., Lovelace, J. K., and Maupin, M. A., 2009, "Estimated use of water in the United States in 2005," USGS Circular 1344, 52 p.

(http://pubs.usgs.gov/circ/1344/). County-level shape files and population density were obtained through the US Census Bureau (http://www.census.gov/geo/mapsdata/data/tiger.html). Water use was summarized by 8 -digit hydrologic unit codes using area-weighted averages of county-level data.

\section{NON-POWERED DAMS}

HSDM: HS_DG: Summary of NPD data components

\begin{tabular}{|c|c|c|c|}
\hline Data_Component & Alias & Type & Description \\
\hline $\begin{array}{l}\text { HS_NPD_Dam } \\
\end{array}$ & Non-Powered Dam & $\begin{array}{l}\text { Geospatial point } \\
\text { layer }\end{array}$ & Geospatial point locations of NPDs $>1 \mathrm{MW}$ \\
\hline HS_NPD_Reservoir & $\begin{array}{l}\text { Non-Powered Dam } \\
\text { Reservoir }\end{array}$ & $\begin{array}{l}\text { Geospatial polygon } \\
\text { layer }\end{array}$ & $\begin{array}{l}\text { Geospatial polygons of reservoirs impounded } \\
\text { by NPDs }>1 \mathrm{MW}\end{array}$ \\
\hline HS_NPD_Tailwater & $\begin{array}{l}\text { Non-Powered Dam } \\
\text { Tailwater }\end{array}$ & $\begin{array}{l}\text { Geospatial polyline } \\
\text { layer }\end{array}$ & $\begin{array}{l}\text { Geospatial polylines of tailwaters below each } \\
\text { NPDs }>1 \text { MW }\end{array}$ \\
\hline
\end{tabular}

HS_NPD_Dam: Attribute summary

\begin{tabular}{|c|c|c|c|}
\hline Alias & Field Name & Unit & Description \\
\hline$\overline{\text { Non-Powerd Dam ID }}$ & NPD_DmID & ID & Unique identification code assigned to each HS_NPD_Dam record \\
\hline $\begin{array}{l}\text { NHDPlus V1 } \\
\text { Common Identifier }\end{array}$ & COMID & ID & NHDPlus V1 common identifier of flow line \\
\hline NID_ID1 & NID_ID1 & ID & $\begin{array}{l}\text { Unique identification code assigned to each dam record within the } \\
\text { NID Database }\end{array}$ \\
\hline Latitude & Lat & DD & Latitude \\
\hline Longitude & Lon & DD & Longitude \\
\hline Dam Name & Dm_Nm & N/A & Name of the dam \\
\hline Dam Owner Name & Dm_Own & N/A & Owner of the dam \\
\hline
\end{tabular}




\begin{tabular}{|c|c|c|c|}
\hline County Name & CountyNm & $\mathrm{N} / \mathrm{A}$ & County the dam is within \\
\hline City Name & CityNm & N/A & City the dam is within \\
\hline $\begin{array}{l}\text { USPS 2-Letter Postal } \\
\text { Code }\end{array}$ & Postal & N/A & $\begin{array}{l}\text { Two-letter US Postal Service abbreviations of US states or } \\
\text { associated territories that each NPD is within }\end{array}$ \\
\hline Watersource & Water & N/A & Primary water source of the dam \\
\hline Year Built & Yr_Built & YYYY & Year dam was built \\
\hline Storage & Storage & ac-ft & $\begin{array}{l}\text { NID Storage. A calculated field based on the maximum value of } \\
\text { maximum storage and normal storage. Maximum storage, in acre- } \\
\text { feet, which is defined as the total storage space in a reservoir below } \\
\text { the maximum attainable water surface elevation, including any } \\
\text { surcharge storage. Normal storage, in acre-feet, which is defined as } \\
\text { the total storage space in a reservoir below the normal retention } \\
\text { level, including dead and inactive storage and excluding any flood } \\
\text { control or surcharge storage. }\end{array}$ \\
\hline $\begin{array}{l}\text { Federal Agency } \\
\text { Regulatory } \\
\text { Involvement }\end{array}$ & FedReg & N/A & $\begin{array}{l}\text { Federal agency involvement in regulation. Code identifying which } \\
\text { federal agency is involved in regulation of the dam. Codes are } \\
\text { concatenated if several agencies are involved. }\end{array}$ \\
\hline Estimated Head & esth & $\mathrm{ft}$ & $\begin{array}{l}\text { Estimated head (ft) available for hydropower generation. Head is } \\
\text { estimated from one of the following: (1) the NID hydraulic height, } \\
\text { (2) } 0.7 \text { times the NID height, (3) US Army Corps of Engineers locks } \\
\text { and dams lift, or (4) the height difference from observed head water } \\
\text { and tailwater elevation. See ORNL NPD Resource Assessment } \\
\text { Report for more details. }\end{array}$ \\
\hline
\end{tabular}
2-Digit HUC HUC_2 ID Watershed boundary dataset 2-digit hydrologic unit region code.
Annual Mean Flow qyr cfs Annual mean flow (cfs). Flow is estimated from either the (1) NHDPlus annual mean flow or (2) drainage area times unit runoff estimated from historic National Water Information System (NWIS)streamflow observation. See ORNL NPD Resource Assessment Report for details.

January Mean Flow q01 cfs

Flow is estimated from either the (1) NHDPlus annual mean flow times ratios estimated from historic NWIS streamflow observation or (2) drainage area times unit runoff estimated from historic NWIS streamflow observation. See ORNL NPD Resource Assessment Report for details.

February Mean Flow q02

cfs Flow is estimated from either the (1) NHDPlus annual mean flow times ratios estimated from historic NWIS streamflow observation or (2) drainage area times unit runoff estimated from historic NWIS streamflow observation. See ORNL NPD Resource Assessment Report for details.

March Mean Flow q03 cfs

Flow is estimated from either the (1) NHDPlus annual mean flow times ratios estimated from historic NWIS streamflow observation or (2) drainage area times unit runoff estimated from historic NWIS 

April Mean Flow
$\mathrm{q} 04$
cfs
Flow is estimated from either the (1) NHDPlus annual mean flow times ratios estimated from historic NWIS streamflow observation or (2) drainage area times unit runoff estimated from historic NWIS streamflow observation. See ORNL NPD Resource Assessment Report for details.

May Mean Flow

q05

June Mean Flow

q06

July Mean Flow

q07

August Mean Flow

q08

September Mean

q09

Flow

October Mean Flow

q10

November Mean

q11

Flow

December Mean

Flow $\mathrm{cfs}$

streamflow observation. See ORNL NPD Resource Assessment Report for details.

Flow is estimated from either the (1) NHDPlus annual mean flow times ratios estimated from historic NWIS streamflow observation or (2) drainage area times unit runoff estimated from historic NWIS streamflow observation. See ORNL NPD Resource Assessment Report for details.

cfs Flow is estimated from either the (1) NHDPlus annual mean flow times ratios estimated from historic NWIS streamflow observation or (2) drainage area times unit runoff estimated from historic NWIS streamflow observation. See ORNL NPD Resource Assessment Report for details.

cfs Flow is estimated from either the (1) NHDPlus annual mean flow times ratios estimated from historic NWIS streamflow observation or (2) drainage area times unit runoff estimated from historic NWIS streamflow observation. See ORNL NPD Resource Assessment Report for details.

cfs Flow is estimated from either the (1) NHDPlus annual mean flow times ratios estimated from historic NWIS streamflow observation or (2) drainage area times unit runoff estimated from historic NWIS streamflow observation. See ORNL NPD Resource Assessment Report for details.

cfs Flow is estimated from either the (1) NHDPlus annual mean flow times ratios estimated from historic NWIS streamflow observation or (2) drainage area times unit runoff estimated from historic NWIS streamflow observation. See ORNL NPD Resource Assessment Report for details.

cfs Flow is estimated from either the (1) NHDPlus annual mean flow times ratios estimated from historic NWIS streamflow observation, or (2) drainage area times unit runoff estimated from historic NWIS streamflow observation. See ORNL NPD Resource Assessment Report for details.

cfs Flow is estimated from either the (1) NHDPlus annual mean flow times ratios estimated from historic NWIS streamflow observation or (2) drainage area times unit runoff estimated from historic NWIS streamflow observation. See ORNL NPD Resource Assessment Report for details.

cfs Flow is estimated from either the (1) NHDPlus annual mean flow times ratios estimated from historic NWIS streamflow observation or (2) drainage area times unit runoff estimated from historic NWIS 
Potential Annual
Hydropower Energy

Potential January mwh01

Hydropower Energy

Potential February mwh02

Hydropower Energy

Potential March mwh03

Hydropower Energy

Potential April mwh04

Hydropower Energy

Potential May mwh05

Hydropower Energy

Potential June mwh06

Hydropower Energy

Potential July mwh07

Hydropower Energy

Potential August mwh08

Hydropower Energy

Potential September mwh09

Hydropower Energy

Potential October mwh10

Hydropower Energy

Potential November mwh11

Hydropower Energy

Potential December mwh12 Hydropower Energy streamflow observation. See ORNL NPD Resource Assessment Report for details.

MWh Potential annual hydropower energy (MWh), a product of estimated flow (cfs), head (ft), efficiency (0.85), time (hr), and 1/11800 for unit conversion.

MWh Potential January hydropower energy (MWh), a product of estimated flow (cfs), head (ft), efficiency (0.85), time (hr), and 1/11800 for unit conversion.

MWh Potential January hydropower energy (MWh), a product of estimated flow (cfs), head (ft), efficiency (0.85), time (hr), and 1/11800 for unit conversion.

MWh Potential January hydropower energy (MWh), a product of estimated flow (cfs), head (ft), efficiency (0.85), time (hr), and 1/11800 for unit conversion.

MWh Potential January hydropower energy (MWh), a product of estimated flow (cfs), head (ft), efficiency (0.85), time (hr), and 1/11800 for unit conversion.

MWh Potential January hydropower energy ( $\mathrm{MWh}$ ), a product of estimated flow (cfs), head (ft), efficiency (0.85), time (hr), and 1/11800 for unit conversion.

MWh Potential January hydropower energy (MWh), a product of estimated flow (cfs), head (ft), efficiency (0.85), time (hr), and 1/11800 for unit conversion.

MWh Potential January hydropower energy ( $\mathrm{MWh}$ ), a product of estimated flow (cfs), head (ft), efficiency (0.85), time (hr), and 1/11800 for unit conversion.

MWh Potential January hydropower energy (MWh), a product of estimated flow (cfs), head (ft), efficiency (0.85), time (hr), and 1/11800 for unit conversion.

MWh Potential January hydropower energy (MWh), a product of estimated flow (cfs), head (ft), efficiency (0.85), time (hr), and 1/11800 for unit conversion.

MWh Potential January hydropower energy (MWh), a product of estimated flow (cfs), head (ft), efficiency (0.85), time (hr), and 1/11800 for unit conversion.

MWh Potential January hydropower energy (MWh), a product of estimated flow (cfs), head (ft), efficiency (0.85), time (hr), and 1/11800 for unit conversion.

MWh Potential January hydropower energy (MWh), a product of estimated flow (cfs), head (ft), efficiency (0.85), time (hr), and 1/11800 for unit conversion. 


\begin{tabular}{|c|c|c|c|}
\hline $\begin{array}{l}\text { Regional Capacity } \\
\text { Factor }\end{array}$ & $\mathrm{cf}$ & $\%$ & $\begin{array}{l}\text { Capacity factor of each power plant is computed by [annual } \\
\text { generation in MWh/(installed capacity in MW } \times 365 \times 24) \text { ]. The } \\
2001-2008 \text { EIA generation and capacity are used to estimate the } \\
\text { capacity factor for each hydropower plant and then weight-averaged } \\
\text { by plant generation to estimate the regional capacity factor. See } \\
\text { ORNL NPD Resource Assessment Report for details. }\end{array}$ \\
\hline Potential Capacity & $\mathrm{mw}$ & MW & $\begin{array}{l}\text { Potential capacity }(\mathrm{MW}) \text {, estimated by [potential annual hydropower } \\
\text { energy in } \mathrm{MWh} /(\mathrm{C} f \times 365 \times 24)] \text {. }\end{array}$ \\
\hline
\end{tabular}

HS_NPD_Reservoir: Attribute summary

\begin{tabular}{|c|c|c|c|}
\hline Alias & Field Name & Unit & Description \\
\hline $\begin{array}{l}\text { Existing Fleet } \\
\text { Reservoir ID }\end{array}$ & NPD_RsID & ID & $\begin{array}{l}\text { Unique identification code assigned to each } \\
\text { HS_EF_Reservoir record. }\end{array}$ \\
\hline Existing Fleet Dam ID & NPD_DmID & ID & $\begin{array}{l}\text { Unique identification code assigned to each } \\
\text { HS_EF_Dam record. }\end{array}$ \\
\hline NID ID & NID_ID1 & ID & $\begin{array}{l}\text { Unique identification code from the US Army Corps of } \\
\text { Engineers National Inventory of Dams for each dam } \\
\text { associated with the reservoir. }\end{array}$ \\
\hline $\begin{array}{l}\text { Dam Distance } \\
\text { Kilometers }\end{array}$ & res_dam_di & $100 \mathrm{~km}$ & $\begin{array}{l}\text { Nearest distance from dam to edge of reservoir. If } \\
\text { intersecting reservoir, then value is } 0 \text {. }\end{array}$ \\
\hline $\begin{array}{l}\text { Area Square } \\
\text { Kilometers }\end{array}$ & AreaSqKm & $\mathrm{km} 2$ & Reservoir area in square kilometers \\
\hline Reservoir Source & res_source & NA & $\begin{array}{l}\text { Source of information used to create the reservoir (either } \\
\text { NHDPlus "Waterbody" or NHDPlus V1 "Area") }\end{array}$ \\
\hline Latitude & lat & $\begin{array}{l}\text { Decimal } \\
\text { degrees }\end{array}$ & Latitude of dam \\
\hline Longitude & lon & $\begin{array}{l}\text { Decimal } \\
\text { degrees }\end{array}$ & Longitude of dam \\
\hline Coordinate From & cordfrom & NA & $\begin{array}{l}\text { Source of information used for the longitude and latitude } \\
\text { of the dam (either NHDPlus V1 or NID) }\end{array}$ \\
\hline Georeferenced Dam & georef_dam & NA & $\begin{array}{l}\text { Indication of whether the dam could be found and } \\
\text { georeferenced (Yes or No) }\end{array}$ \\
\hline
\end{tabular}


HS_NPD_Tailwater: Attribute summary

\begin{tabular}{|c|c|c|c|}
\hline Alias & Field Name & Unit & Description \\
\hline $\begin{array}{l}\text { NPD Tailwater } \\
\text { ID }\end{array}$ & NPD_TIID & ID & $\begin{array}{l}\text { Unique identification code assigned to each } \\
\text { HS_NPD_Tailwater record }\end{array}$ \\
\hline $\begin{array}{l}\text { Non-Powerd } \\
\text { Dam ID }\end{array}$ & NPD_DmID & ID & $\begin{array}{l}\text { Unique identification code assigned to each HS_NPD_Dam } \\
\text { record }\end{array}$ \\
\hline \multirow[t]{2}{*}{ NID ID } & NID_ID & ID & $\begin{array}{l}\text { Unique identification code from the US Army Corps of } \\
\text { Engineers NID for each dam associated with the tailwater }\end{array}$ \\
\hline & Water & & \\
\hline Latitude & Lat & Decimal degrees & Latitude of dam \\
\hline Longitude & Lon & Decimal degrees & Longitude of dam \\
\hline Length $\mathrm{Km}$ & LENGTHKM & Kilometers & Total length of each tailwater in kilometers \\
\hline COMID Initial & COMIDini & ID & $\begin{array}{l}\text { Unique identification code from NHDPlus V1 for each stream } \\
\text { reach at the upstream end of the tailwater }\end{array}$ \\
\hline COMID End & COMIDend & ID & $\begin{array}{l}\text { Unique identification code from NHDPlus V1 for each stream } \\
\text { reach at the downstream end of the tailwater }\end{array}$ \\
\hline COMID & COMID & ID & $\begin{array}{l}\text { Unique identification code from NHDPlus V1 for each stream } \\
\text { reach at the location of the dam }\end{array}$ \\
\hline
\end{tabular}

7. NEW STREAM-REACH DEVELOPMENT

HSDM: HS_DG: Summary of NSD data components

\begin{tabular}{|c|c|c|c|}
\hline Data_Component & Alias & Type & Description \\
\hline HS_NSD_StreamReach & $\begin{array}{l}\text { New Stream-Reach } \\
\text { Development }\end{array}$ & $\begin{array}{l}\text { Geospatial point } \\
\text { layer }\end{array}$ & $\begin{array}{l}\text { Geospatial point locations of NSD } \\
\text { potential within stream reaches }\end{array}$ \\
\hline HS_NSD_Inundation & $\begin{array}{l}\text { New Stream-Reach } \\
\text { Development Inundated } \\
\text { Area }\end{array}$ & $\begin{array}{l}\text { Geospatial } \\
\text { polygon layer }\end{array}$ & $\begin{array}{l}\text { Geospatial polygons of reservoirs } \\
\text { impounded by locations of NSD stream } \\
\text { reaches within the HydroSource } \\
\text { Database }\end{array}$ \\
\hline HS_NSD_Tailwater & $\begin{array}{l}\text { New Stream-Reach } \\
\text { Development Tailwater }\end{array}$ & $\begin{array}{l}\text { Geospatial } \\
\text { polyline layer }\end{array}$ & $\begin{array}{l}\text { Geospatial polylines of tailwaters below } \\
\text { each NSD stream reach within the } \\
\text { HydroSource Database }\end{array}$ \\
\hline HS_NSD_Environmental & $\begin{array}{l}\text { NSD Environmental } \\
\text { Attributes }\end{array}$ & Nonspatial table & $\begin{array}{l}\text { Environmental attributes geospatially } \\
\text { associated with the NSD inundated area, } \\
\text { NSD stream reach, and NSD tailwater } \\
\text { layers within the HydroSource Database }\end{array}$ \\
\hline
\end{tabular}


HS_NSD_StreamReach: Attribute summary

\begin{tabular}{|c|c|c|c|}
\hline Alias & Field Name & Unit & Description \\
\hline NSD Reach ID & ReachID & ID & $\begin{array}{l}\text { The unique stream-reach identifier used in the NSD assessment. } \\
\text { The last 4-digits of ReachID is the Subregion HUC04 ID. }\end{array}$ \\
\hline 10-Digit HUC & HUC_10 & ID & $\begin{array}{l}\text { Ten-digit HUC watershed identifier from the Watershed Boundary } \\
\text { Dataset. }\end{array}$ \\
\hline State Name & State & $\mathrm{N} / \mathrm{A}$ & State name. \\
\hline \multirow[t]{2}{*}{$\begin{array}{l}\text { NHDPlus V1 Common } \\
\text { Identifier }\end{array}$} & COMID & ID & NHDPlus V1 flow line common identifier. \\
\hline & GNIS_NAME & N/A & The GNIS river name provided by the NHD. \\
\hline NSD Dam Longitude & DamLon & DD & Longitude of the potential dam. \\
\hline NSD Dam Latitude & DamLat & DD & Latitude of the potential dam. \\
\hline \multirow[t]{2}{*}{ NSD Dam Height } & DamH_m & $\mathrm{m}$ & $\begin{array}{l}\text { Potential dam height (or hydraulic head), estimated by reference } \\
\text { height calculated from the Federal Emergency Management } \\
\text { Agency (FEMA) 100-year flood elevation. }\end{array}$ \\
\hline & DamH_ft & $\mathrm{ft}$ & $\begin{array}{l}\text { Potential dam height (or hydraulic head), estimated by reference } \\
\text { height calculated from the FEMA 100-year flood elevation. }\end{array}$ \\
\hline Tailwater Elevation & Tail_m & $\mathrm{m}$ & Tailwater elevation. \\
\hline Pool Water Elevation & Pool_m & $\mathrm{m}$ & Pool water elevation. \\
\hline Reservoir Length & Length_m & $\mathrm{m}$ & The total reservoir length. \\
\hline $\begin{array}{l}\text { Average Channel } \\
\text { Slope }\end{array}$ & Slope & $\mathrm{m}$ & Average channel slope (DamH_m/Length_m). \\
\hline Annual Mean Flow & QNHD_cfs & $\mathrm{cfs}$ & $\begin{array}{l}\text { The annual mean flow provided by NHDPlus (estimated by the } \\
\text { unit runoff method). }\end{array}$ \\
\hline Flow Adjustment Ratio & Q_ratio & N/A & $\begin{array}{l}\text { Flow adjustment ratio used to estimate the NSD hydraulic capacity } \\
\text { from NHDPlus version } 1 \text { flow (Q30= Q_ratio } \times \text { QNHD_cfs), } \\
\text { derived from the USGS NWIS gauge observation. }\end{array}$ \\
\hline \multirow[t]{2}{*}{ Hydraulic Capacity } & Q30_cfs & $\mathrm{cfs}$ & $\begin{array}{l}\text { Hydraulic capacity, defined by the } 30 \% \text { exceedance quantile from } \\
\text { the flow-duration curve, estimated by } \mathrm{Q} 30=\mathrm{Q} \text { ratio } \times \text { QNHD_cfs. }\end{array}$ \\
\hline & HQS & & The product of DamH_m, QNHD_cfs, and slope. \\
\hline Generating Efficiency & eff & $\%$ & Generating efficiency, assumed to be 0.85 in this study. \\
\hline $\begin{array}{l}\text { Potential Hydropower } \\
\text { Capacity }\end{array}$ & PNSD_MW & MW & $\begin{array}{l}\text { Total potential capacity from identified stream reaches with } \\
\text { capacity greater than } 1 \mathrm{MW} \text {. }\end{array}$ \\
\hline Annual Mean Flow & QYR_cfs & $\mathrm{cfs}$ & $\begin{array}{l}\text { Annual mean flow estimated by the drainage area and USGS } \\
\text { WaterWatch runoff }\end{array}$ \\
\hline
\end{tabular}




\begin{tabular}{|c|c|c|c|}
\hline January Mean Flow & QJan_cfs & $\mathrm{cfs}$ & $\begin{array}{l}\text { January mean flow estimated by the drainage area and USGS } \\
\text { WaterWatch runoff. }\end{array}$ \\
\hline February Mean Flow & QFeb_cfs & $\mathrm{cfs}$ & $\begin{array}{l}\text { February mean flow estimated by the drainage area and USGS } \\
\text { WaterWatch runoff. }\end{array}$ \\
\hline March Mean Flow & QMar_cfs & $\mathrm{cfs}$ & $\begin{array}{l}\text { March mean flow estimated by the drainage area and USGS } \\
\text { WaterWatch runoff. }\end{array}$ \\
\hline April Mean Flow & QApr_cfs & $\mathrm{cfs}$ & $\begin{array}{l}\text { April mean flow estimated by the drainage area and USGS } \\
\text { WaterWatch runoff. }\end{array}$ \\
\hline May Mean Flow & QMay_cfs & $\mathrm{cfs}$ & $\begin{array}{l}\text { May mean flow estimated by the drainage area and USGS } \\
\text { WaterWatch runoff. }\end{array}$ \\
\hline June Mean Flow & QJun_cfs & $\mathrm{cfs}$ & $\begin{array}{l}\text { June mean flow estimated by the drainage area and USGS } \\
\text { WaterWatch runoff. }\end{array}$ \\
\hline July Mean Flow & QJul_cfs & $\mathrm{cfs}$ & $\begin{array}{l}\text { July mean flow estimated by the drainage area and USGS } \\
\text { WaterWatch runoff. }\end{array}$ \\
\hline August Mean Flow & QAug_cfs & $\mathrm{cfs}$ & $\begin{array}{l}\text { August mean flow estimated by the drainage area and USGS } \\
\text { WaterWatch runoff. }\end{array}$ \\
\hline September Mean Flow & QSep_cfs & $\mathrm{cfs}$ & $\begin{array}{l}\text { September mean flow estimated by the drainage area and USGS } \\
\text { WaterWatch runoff. }\end{array}$ \\
\hline October Mean Flow & QOct_cfs & $\mathrm{cfs}$ & $\begin{array}{l}\text { October mean flow estimated by the drainage area and USGS } \\
\text { WaterWatch runoff. }\end{array}$ \\
\hline November Mean Flow & QNov_cfs & $\mathrm{cfs}$ & $\begin{array}{l}\text { November mean flow estimated by the drainage area and USGS } \\
\text { WaterWatch runoff. }\end{array}$ \\
\hline December Mean Flow & QDec_cfs & $\mathrm{cfs}$ & $\begin{array}{l}\text { December mean flow estimated by the drainage area and USGS } \\
\text { WaterWatch runoff. }\end{array}$ \\
\hline $\begin{array}{l}\text { Estimated Potential } \\
\text { Annual Energy }\end{array}$ & ENSD_MWh & MWh & $\begin{array}{l}\text { Estimated potential annual energy at stream reaches. See NSD } \\
\text { Methodology Report for more details. }\end{array}$ \\
\hline $\begin{array}{l}\text { Estimated Potential } \\
\text { January Energy }\end{array}$ & EJan_MWh & MWh & $\begin{array}{l}\text { Estimated potential January energy at stream reaches. See NSD } \\
\text { Methodology Report for more details. }\end{array}$ \\
\hline $\begin{array}{l}\text { Estimated Potential } \\
\text { February Energy }\end{array}$ & EFeb_MWh & MWh & $\begin{array}{l}\text { Estimated potential February energy at stream reaches. See NSD } \\
\text { Methodology Report for more details. }\end{array}$ \\
\hline $\begin{array}{l}\text { Estimated Potential } \\
\text { March Energy }\end{array}$ & EMar_MWh & MWh & $\begin{array}{l}\text { Estimated potential March energy at stream reaches. See NSD } \\
\text { Methodology Report for more details. }\end{array}$ \\
\hline $\begin{array}{l}\text { Estimated Potential } \\
\text { April Energy }\end{array}$ & EApr_MWh & MWh & $\begin{array}{l}\text { Estimated potential April energy at stream reaches. See NSD } \\
\text { Methodology Report for more details. }\end{array}$ \\
\hline $\begin{array}{l}\text { Estimated Potential } \\
\text { May Energy }\end{array}$ & EMay_MWh & MWh & $\begin{array}{l}\text { Estimated potential May energy at stream reaches. See NSD } \\
\text { Methodology Report for more details. }\end{array}$ \\
\hline
\end{tabular}




\begin{tabular}{|c|c|c|c|}
\hline $\begin{array}{l}\text { Estimated Potential } \\
\text { June Energy }\end{array}$ & EJun_MWh & MWh & $\begin{array}{l}\text { Estimated potential June energy at stream reaches. See NSD } \\
\text { Methodology Report for more details. }\end{array}$ \\
\hline $\begin{array}{l}\text { Estimated Potential } \\
\text { July Energy }\end{array}$ & EJul_MWh & MWh & $\begin{array}{l}\text { Estimated potential July energy at stream reaches. See NSD } \\
\text { Methodology Report for more details. }\end{array}$ \\
\hline $\begin{array}{l}\text { Estimated Potential } \\
\text { August Energy }\end{array}$ & EAug_MWh & MWh & $\begin{array}{l}\text { Estimated potential August energy at stream reaches. See NSD } \\
\text { Methodology Report for more details. }\end{array}$ \\
\hline $\begin{array}{l}\text { Estimated Potential } \\
\text { September Energy }\end{array}$ & ESep_MWh & MWh & $\begin{array}{l}\text { Estimated potential September energy at stream reaches. See NSD } \\
\text { Methodology Report for more details. }\end{array}$ \\
\hline $\begin{array}{l}\text { Estimated Potential } \\
\text { October Energy }\end{array}$ & EOct_MWh & MWh & $\begin{array}{l}\text { Estimated potential October energy at stream reaches. See NSD } \\
\text { Methodology Report for more details. }\end{array}$ \\
\hline $\begin{array}{l}\text { Estimated Potential } \\
\text { November Energy }\end{array}$ & ENov_MWh & MWh & $\begin{array}{l}\text { Estimated potential November energy at stream reaches. See NSD } \\
\text { Methodology Report for more details. }\end{array}$ \\
\hline $\begin{array}{l}\text { Estimated Potential } \\
\text { December Energy }\end{array}$ & EDec_MWh & MWh & $\begin{array}{l}\text { Estimated potential December energy at stream reaches. See NSD } \\
\text { Methodology Report for more details. }\end{array}$ \\
\hline $\begin{array}{l}\text { Annual Capacity } \\
\text { Factor }\end{array}$ & Cf_yr & $\%$ & $\begin{array}{l}\text { Capacity factor, estimated by (potential annual energy in } \\
\mathrm{MWh}) /(\text { potential capacity in } \mathrm{MW} \times 365 \times 24) \text {. }\end{array}$ \\
\hline Spring Capacity Facto & Cf_Sp & $\%$ & $\begin{array}{l}\text { Capacity factor, estimated by (potential March-May energy in } \\
\mathrm{MWh}) /(\text { potential capacity in } \mathrm{MW} \times(31+30+31) \times 24) \text {. }\end{array}$ \\
\hline $\begin{array}{l}\text { Summer Capacity } \\
\text { Factor }\end{array}$ & Cf_Su & $\%$ & $\begin{array}{l}\text { Capacity factor, estimated by (potential June-August energy in } \\
\mathrm{MWh}) /(\text { potential capacity in } \mathrm{MW} \times(30+31+31) \times 24 \text {. }\end{array}$ \\
\hline Fall Capacity Factor & $\mathrm{Cf} \_\mathrm{Fa}$ & $\%$ & $\begin{array}{l}\text { Capacity factor, estimated by (potential September-November } \\
\text { energy in } \mathrm{MWh}) /(\text { potential capacity in } \mathrm{MW} \times(30+31+30) \times 24) \text {. }\end{array}$ \\
\hline Winter Capacity Facto & Cf_Wi & $\%$ & $\begin{array}{l}\text { Capacity factor, estimated by (potential December-February } \\
\text { energy in } \mathrm{MWh}) /(\text { potential capacity in } \mathrm{MW} \times(31+31+28) \times 24) \text {. }\end{array}$ \\
\hline $\begin{array}{l}\text { Number of Pipes in } \\
\text { Diversion Scenario }\end{array}$ & Div_NPipe & Number & $\begin{array}{l}\text { Number of pipes calculated in the diversion scenario. See NSD } \\
\text { Methodology Report for more details. }\end{array}$ \\
\hline $\begin{array}{l}\text { Diameter of Pipes in } \\
\text { Diversion Scenario }\end{array}$ & Div_D_ft & $\mathrm{ft}$ & $\begin{array}{l}\text { Diameter of the pipes calculated in the diversion scenario. See } \\
\text { NSD Methodology Report for more details. }\end{array}$ \\
\hline $\begin{array}{l}\text { Velocity of Pipes in } \\
\text { Diversion Scenario }\end{array}$ & Div_V_fts & $\mathrm{ft} / \mathrm{s}$ & $\begin{array}{l}\text { Velocity in the pipes }(\mathrm{ft} / \mathrm{s}) \text { calculated in the diversion scenario. See } \\
\text { NSD Methodology Report for more details. }\end{array}$ \\
\hline Effective Head & Div_H_ft & $\mathrm{ft}$ & $\begin{array}{l}\text { Effective head (after considering head loss) calculated in the } \\
\text { diversion scenario. See NSD Methodology Report for more details. }\end{array}$ \\
\hline $\begin{array}{l}\text { Diversion Scenario } \\
\text { Potential Capacity }\end{array}$ & Div_P_MW & MW & $\begin{array}{l}\text { Potential capacity calculated in the diversion scenario. See NSD } \\
\text { Methodology Report for more details. }\end{array}$ \\
\hline
\end{tabular}


HS_NSD_Inundation: Attribute summary

\begin{tabular}{|c|c|c|c|}
\hline Alias & Field Name & Unit & Description \\
\hline $\begin{array}{l}\text { NSD } \\
\text { Inundation } \\
\text { ID }\end{array}$ & NSD_InID & ID & Unique identification code assigned to each HS_NSD_Inundation record. \\
\hline $\begin{array}{l}\text { NSD Reach } \\
\text { ID }\end{array}$ & ReachID & ID & $\begin{array}{l}\text { Unique stream-reach identifier used in the NSD assessment. The last 4- } \\
\text { digits of ReachID is the Subregion HUC04 ID. }\end{array}$ \\
\hline $\begin{array}{l}\text { Estimated } \\
\text { Surface } \\
\text { Inundation }\end{array}$ & ANSD_ac & ac & $\begin{array}{l}\text { Estimated surface inundation corresponding to the reference height, } \\
\text { derived from the 10-meter resolution National Elevation Datase. }\end{array}$ \\
\hline $\begin{array}{l}\text { Estimated } \\
\text { Reservoir } \\
\text { Storage }\end{array}$ & VNSD_acft & ac-ft & $\begin{array}{l}\text { Estimated reservoir storage corresponding to the reference height, derived } \\
\text { from the } 10 \mathrm{~m} \text { resolution National Elevation Dataset. }\end{array}$ \\
\hline $\begin{array}{l}\text { Residence } \\
\text { Time }\end{array}$ & TNSD_day & & $\begin{array}{l}\text { Residence time, estimated by } \mathrm{d} \times(\text { reservoir storage in ac } \times \mathrm{ft}) /(\mathrm{NHDPlus} \\
\text { annual mean flow in } \mathrm{cfs}) . \mathrm{d}=0.504 \text { is a unit conversion factor. }\end{array}$ \\
\hline \multirow[t]{5}{*}{$\begin{array}{l}\text { Critical } \\
\text { Habitats }\end{array}$} & CRITHABi & Number & $\begin{array}{l}\text { Number of critical habitats occurring within } 800 \mathrm{~m} \text { of potential inundated } \\
\text { areas designated for potential hydropower development. Critical habitats } \\
\text { are lines or polygons designated by the US Fish and Wildlife Service for } \\
\text { federally listed species under the ESA of } 1973 \text {. }\end{array}$ \\
\hline & FISHESAi & Number & $\begin{array}{l}\text { Number of federally listed fish species potentially occurring within the 8- } \\
\text { HUC watershed containing each inundated area. Federally listed fish } \\
\text { included species whose entire population or subpopulation is listed as } \\
\text { "endangered," "threatened," proposed for listing, or a species of concern } \\
\text { according to the ESA (1973) (http://www.fws.gov/endangered/species/us- } \\
\text { species.html). Fish species distributions were available from NatureServe } \\
\text { (http://www.natureserve.org/getData/fishMaps.jsp). Federally listed } \\
\text { species were obtained from the US Fish and Wildlife Service. }\end{array}$ \\
\hline & FSHIUCNi & Number & $\begin{array}{l}\text { Number of fish species of concern potentially occurring within the 8-HUC } \\
\text { watershed containing each inundated area. Fish species of concern were } \\
\text { determined according to the IUCN Red List Criteria } \\
\text { (http://www.iucnredlist.org/technical-documents/categories-and-criteria). } \\
\text { Fish species distributions were available from NatureServe at the 8-HUC } \\
\text { watershed scale (http://www.natureserve.org/getData/fishMaps.jsp). } \\
\text { IUCN rankings were obtained from NatureServe explorer } \\
\text { (www.natureserve.org/explorer/). }\end{array}$ \\
\hline & FISHPOTi & Number & $\begin{array}{l}\text { Number of fish species documented as potadromous within the } 8 \text {-HUC } \\
\text { watershed containing each inundated area. Potadromous fish are species } \\
\text { migrating entirely in freshwater to complete their life cycle requirements. }\end{array}$ \\
\hline & PROTLNDi & Number & $\begin{array}{l}\text { An indication of whether protected conservation lands occur within } 2,500 \\
\text { m of potential inundated areas designated for potential hydropower } \\
\text { development. "1" indicating "yes," and "0" indicating "no." Protected }\end{array}$ \\
\hline
\end{tabular}


LOWNIDXi Number

LNDDESGi Number

NATPRKi Number

WSRi Number

303d Listed Wqi

Waterbodies

Whitewater AWRAFTi

Boating

Runs

Number conservation lands were obtained from the Protected Area Database, developed by the USGS Gap Analysis Program.

The Land Ownership Index is the sum of the number of different governmental or nongovernmental entity lands that occur within $2,500 \mathrm{~m}$ of potential inundated areas designated for potential hydropower development.

The Land Designation Index is the sum of the number of different land designations that occur within $2,500 \mathrm{~m}$ of potential inundated areas designated for potential hydropower development. Land designations include US National Park, US National Forest/Grassland, National Trail, National Wildlife Refuge, National Natural Landmark, National Landscape Conservation System (Non-Wilderness), National Landscape Conservation System (Wilderness), Wild and Scenic Rivers, Marine Protected Area, Wilderness Area, Historic/Cultural Area, Military Land, Habitat or Species Management Area, Research Natural Area, Local Conservation, Private Conservation, and Conservation Program Land. Thus, the Land Designation Index could range from 0 (no protected land intersecting a stream reach) to 17 (all designation types intersect a stream reach). Protected conservation lands were obtained from the Protected Area Database, developed by the USGS Gap Analysis Program.

An indication of whether US National Parks occur within 2,500 m of potential inundated areas designated for potential hydropower development. "1" indicating "yes," and " 0 " indicating "no." US National Park lands were obtained from the Protected Area Database developed by the USGS Gap Analysis Program (http://gapanalysis.usgs.gov/data/padusdata/padus-data-download/).

An indication of whether US designated Wild and Scenic Rivers occur within 2,500 $\mathrm{m}$ of potential inundated areas designated for potential hydropower development. "1" indicating "yes," and "0" indicating "no." Wild and Scenic River polylines were obtained from the National Wild and Scenic Rivers System website (http://www.rivers.gov/rivers/mappinggis.php).

Number Number of $303 \mathrm{~d}$ listed waterbodies occurring within $500 \mathrm{~m}$ of potential inundated areas designated for potential hydropower development. Under the Clean Water Act (1977), Section 303(d), states are required to specify designated uses for all waterways (e.g., public water supply, protection of fish and wildlife, recreation). In addition, each state must identify and adopt water quality criteria that support each designated use category and determine a list of streams that are not meeting their designated uses (303d List). 303d waterbody listings were obtained from the US EPA Impaired Waters and Total Maximum Daily Load website (http://www.epa.gov/waters/data/downloads.html). Number of whitewater boating runs (launch/take-out point combinations) occurring within $500 \mathrm{~m}$ of potential inundated areas designated for potential hydropower development. Locations of whitewater boating runs 


$\begin{array}{llll}\text { Waterfalls } & \text { FALLSi } & \text { Number } & \begin{array}{l}\text { Number of waterfalls occurring within } 800 \mathrm{~m} \text { of potential inundated areas } \\ \text { designated for potential hydropower development. Locations of waterfalls } \\ \text { were obtained from Geology.com (http://geology.com/waterfalls/). }\end{array} \\ \text { Boat } & \text { BOATRMPi } & \text { Number } & \begin{array}{l}\text { Average number of developed and undeveloped boat ramps intersecting } \\ \text { Ramps }\end{array} \\ & & \begin{array}{l}500 \mathrm{~m} \text { buffered areas around potential inundated areas for development } \\ \text { within each } 10 \text {-HUC watershed. Locations of boat ramps were obtained } \\ \text { from Delorme Publishing Company (http://www.delorme.com/). }\end{array}\end{array}$

were obtained from the American Whitewater National Whitewater Inventory (http://www.americanwhitewater.org/content/River/view/).

FISHACCi Number Average number of fishing access locations intersecting buffered areas around stream-reaches designated for potential development within each 10-HUC watershed. Boat ramp locations were obtained from Delorme Publishing Company (http://www.delorme.com/).

S_WUSEi Estimated surface water use $\left[(\mathrm{L} / \mathrm{d}) / \mathrm{km}^{2}\right]$ within the 8 -HUC watershed containing each inundated area. Estimates of 2005 water use in the United States were obtained from the USGS Water Use Data site (http://water.usgs.gov/watuse/data/2005/index.html). Estimates per 8HUC watersheds were based on area-weighted averages from county estimates.

G_WUSEi Estimated groundwater use $\left[(\mathrm{L} / \mathrm{d}) / \mathrm{km}^{2}\right]$ within the 8 -HUC watershed containing each inundated area. Estimates of 2005 water use in the United States were obtained from the USGS Water Use Data site (http://water.usgs.gov/watuse/data/2005/index.html). Estimates per 8HUC watersheds were based on area-weighted averages from county estimates.

Catchment URBANi \% Urbanizatio Percentage of area composed of low-intensity, moderate-intensity, and high-intensity urbanization within each NHDPlus watershed containing each inundated area. Urban landcover based on National Land Cover Database 2001 (Multi-Resolution Land Characteristics Consortium [MRLC]: http://www.mrlc.gov/datasets). Urban land cover within each NHDPlus catchment was available from the National Fish Habitat Action Plan dataset (http://ecosystems.usgs.gov/fishhabitat/).

Catchment POPDENi Population Estimated population density (individuals $/ \mathrm{km}^{2}$ ) within each NHDPlus watershed containing each inundated area. Based on US Population Density 2000 data from the National Oceanic and Atmospheric Administration: http://www.ngdc.noaa.gov/dmsp/download_sprawl.html. Population density within each NHDPlus catchment was available from the National Fish Habitat Action Plan dataset (http://ecosystems.usgs.gov/fishhabitat/).

L_DAMSi Number Number of dams occurring within each NHDPlus watershed containing each inundated area. Dam locations were based on the US Army Corps of Engineers National Dam Inventory (http://www.usace.army.mil/Library/Maps/Pages/NationalInventoryofDa ms.aspx). The number of dams per NHDPlus catchment was available 
from the National Fish Habitat Action Plan dataset (http://ecosystems.usgs.gov/fishhabitat/).

Number of N_DAMSi Number Number of dams occurring in the drainage network upstream of each Upstream NHDPlus watershed containing each inundated area. Dam locations based on the US Army Corps of Engineers National Dam Inventory (http://www.usace.army.mil/Library/Maps/Pages/NationalInventoryofDa ms.aspx). The number of dams occurring in the entire upstream network per each NHDPlus catchment was available from the National Fish Habitat Action Plan dataset (http://ecosystems.usgs.gov/fishhabitat/).

Inundated LD_IDXi Number The Land Disturbance Index for each NHDPlus watershed containing

Area Land each inundated area. Land Disturbance Indices were created for NHDPlus catchments through the National Fish Habitat Action Plan

Disturbance (http://ecosystems.usgs.gov/fishhabitat/). A cumulative disturbance index was created for approximately 2.23 million US river reaches (NHD flow lines) from landscape anthropogenic activities using urban areas, agricultural areas, roads, dams, mines, population density, and pointsource pollution sites. Lower values indicate higher disturbance to aquatic habitats.

HS_NSD_Tailwater: Attribute summary

\begin{tabular}{|c|c|c|c|}
\hline Alias & Field Name & Unit & Description \\
\hline $\begin{array}{l}\text { NSD } \\
\text { Tailwater ID }\end{array}$ & NSD_TIID & ID & Unique identification code assigned to each HS_NSD_Tailwater record. \\
\hline $\begin{array}{l}\text { NSD Reach } \\
\text { ID }\end{array}$ & ReachID & ID & $\begin{array}{l}\text { Unique stream-reach identifier used in the NSD assessment. The last 4-digits of } \\
\text { ReachID is the Subregion HUC04 ID. }\end{array}$ \\
\hline $\begin{array}{l}\text { 10-Digit } \\
\text { HUC }\end{array}$ & HUC_10 & ID & Ten-digit HUC watershed identifier from the Watershed Boundary Dataset. \\
\hline $\begin{array}{l}\text { COMID } \\
\text { Initial }\end{array}$ & COMIDini & ID & $\begin{array}{l}\text { Unique identification code from NHDPlus V1 for each stream reach at the } \\
\text { upstream end of the tailwater. }\end{array}$ \\
\hline COMID End & COMIDend & ID & $\begin{array}{l}\text { Unique identification code from NHDPlus V1 for each stream reach at the } \\
\text { downstream end of the tailwater. }\end{array}$ \\
\hline $\begin{array}{l}\text { GNIS River } \\
\text { Name }\end{array}$ & \multicolumn{2}{|c|}{ GNIS_NAME ID } & The GNIS river name provided by the NHD. \\
\hline $\begin{array}{l}\text { Tailwater } \\
\text { Length }\end{array}$ & \multicolumn{2}{|c|}{ LENGTHKM km } & $\begin{array}{l}\text { Tailwater length, defined by calculating the total length of all merged NHDPlus } \\
\text { V1 flow lines comprising the tailwater. }\end{array}$ \\
\hline $\begin{array}{l}\text { Potential } \\
\text { Dam } \\
\text { Longitude }\end{array}$ & DamLon & DD & Longitude of the potential dam. \\
\hline
\end{tabular}


Potential DamLat DD Latitude of the potential dam.

Dam

Latitude

Critical CRITHABt Number Number of critical habitats occurring within $800 \mathrm{~m}$ of each tailwater. Critical

Habitat habitats are lines or polygons designated by the US Fish and Wildlife Service for federally listed species under the ESA of 1973

(http://criticalhabitat.fws.gov/crithab/).

Federally FISHESAt Number Number of federally listed fish species potentially occurring within the 8-HUC Listed Fish watershed containing each tailwater. Federally listed fish included species whose entire population or subpopulation is listed as "endangered," "threatened," proposed for listing, or a species of concern according to the ESA (1973) (http://www.fws.gov/endangered/species/us-species.html). Fish species distributions were available from NatureServe (http://www.natureserve.org/getData/fishMaps.jsp). Federally listed species were obtained from the US Fish and Wildlife Service.

Fish Species FISHIUCNt of Concern

Number Number of fish species of concern potentially occurring within the 8-HUC watershed containing each tailwater. Fish species of concern were determined according to the IUCN Red List Criteria (http://www.iucnredlist.org/technicaldocuments/categories-and-criteria). Fish species distributions were available from NatureServe at the 8-HUC watershed scale (http://www.natureserve.org/getData/fishMaps.jsp). IUCN rankings were obtained from NatureServe explorer (www.natureserve.org/explorer/).

Potadromous FISHPOTt Number Number of fish species documented as potadromous within the 8-HUC Fish Species watershed containing each tailwater. Potadromous fish are species migrating entirely in freshwater to complete their life cycle requirements. Fish species distributions were available from NatureServe at the 8-HUC watershed scale (http://www.natureserve.org/getData/fishMaps.jsp). Information on potadromy for fishes was obtained through the Fish Traits Database (http://fishwild.vt.edu/fishtraits/). See also Frimpong, E. A. and P. L. Angermeier (2009), "Fish Traits: A database of Ecological and Life-history traits of freshwater fishes of the United States," Fisheries 34: 487-495.

Protected PROTLNDt Number An indication of whether protected conservation lands occur within $800 \mathrm{~m}$ of Lands each tailwater. "1" indicating "yes," and " 0 " indicating "no." Protected conservation lands were obtained from the Protected Area Database, developed by the USGS Gap Analysis Program.

Land LOWNIDXt Number The Land Ownership Index is the sum of the number of different governmental Ownership or nongovernmental entity lands that occur within $800 \mathrm{~m}$ of each tailwater. Entities include federal, state, nongovernmental agency, local government, Native American, regional agency, territorial, private conservation, joint ownership, and unknown agency. Thus, the Land Ownership Index could range from 0 (no protected land intersecting a stream reach) to 10 (all entities have lands intersecting a stream reach). Protected conservation lands were obtained from the Protected Area Database developed by the USGS Gap Analysis Program. 
Land LNDDESGt Number The Land Designation Index is the sum of the number of different land Designation

Index designations that occur within $80 \mathrm{~m}$ of each tailwater. Land designations include US National Park, US National Forest/Grassland, National Trail, National Wildlife Refuge, National Natural Landmark, National Landscape Conservation System (Non-Wilderness), National Landscape Conservation System (Wilderness), Wild and Scenic Rivers, Marine Protected Area, Wilderness Area, Historic/Cultural Area, Military Land, Habitat or Species Management Area, Research Natural Area, Local Conservation, Private Conservation, and Conservation Program Land. Thus, the Land Designation Index could range from 0 (no protected land intersecting a stream reach) to 17 (all designation types intersect a stream reach). Protected conservation lands were obtained from the Protected Area Database developed by the USGS Gap Analysis Program (http://gapanalysis.usgs.gov/data/padus-data/padus-datadownload/).

National NATPRKt Number An indication of whether US National Parks occur within $800 \mathrm{~m}$ of each Parks tailwater. " 1 " indicating "yes," and "0" indicating "no." US National Park lands were obtained from the Protected Area Database developed by the USGS Gap Analysis Program (http://gapanalysis.usgs.gov/data/padus-data/padusdata-download/).

Wild and WSRt Scenic Number An indication of whether US designated Wild and Scenic Rivers occur within $800 \mathrm{~m}$ of each tailwater. " 1 " indicating "yes," and "0" indicating "no." Wild and Scenic River polylines were obtained from the National Wild and Scenic Rivers System website (http://www.rivers.gov/rivers/mapping-gis.php).

303d Listed WQt

Waterbodies

Number Number of $303 \mathrm{~d}$ listed waterbodies occurring within $500 \mathrm{~m}$ of each tailwater. Under the Clean Water Act (1977), Section 303(d), states are required to specify designated uses for all waterways (e.g., public water supply, protection of fish and wildlife, recreation). In addition, each state must identify and adopt water quality criteria that support each designated use category and determine a list of streams that are not meeting their designated uses (303d List). 303d waterbody listings were obtained from the US EPA Impaired Waters and Total Maximum Daily Load website (http://www.epa.gov/waters/data/downloads.html).

Waterfalls FALLSt Number Number of waterfalls occurring within $800 \mathrm{~m}$ of each tailwater. Locations of waterfalls were obtained from Geology.com (http://geology.com/waterfalls/).

Developed BOATRMPt Number Number of developed and undeveloped boat ramps occurring within $500 \mathrm{~m}$ of and

Undeveloped each tailwater. Locations of boat ramps were obtained from Delorme Publishing Company (http://www.delorme.com/).

Boat Ramps

Fishing

FISHACCt

Access

Locations

Estimated S_WUSEt

Surface

Water Use

Number Number of fishing access locations occurring within $500 \mathrm{~m}$ of each tailwater. Boat ramp locations were obtained from Delorme Publishing Company (http://www.delorme.com/).

Estimated surface water use $\left[(\mathrm{L} / \mathrm{d}) / \mathrm{km}^{2}\right]$ within the 8 -HUC watershed containing each tailwater. Estimates of 2005 water use in the United States were obtained from the USGS Water Use Data site (http://water.usgs.gov/watuse/data/2005/index.html). Estimates per 8-HUC watersheds were based on area-weighted averages from county estimates. 


\begin{tabular}{|c|c|c|c|}
\hline $\begin{array}{l}\text { Estimated } \\
\text { Ground } \\
\text { Water Use }\end{array}$ & G_WUSEt & & $\begin{array}{l}\text { Estimated ground water use }\left[(\mathrm{L} / \mathrm{d}) / \mathrm{km}^{2}\right] \text { within the } 8 \text {-HUC watershed } \\
\text { containing each tailwater. Estimates of } 2005 \text { water use in the United States } \\
\text { were obtained from the Water Use Data site } \\
\text { (http://water.usgs.gov/watuse/data/2005/index.html). Estimates per 8-HUC } \\
\text { watersheds were based on area-weighted averages from county estimates. }\end{array}$ \\
\hline Urbanization & URBANt & $\%$ & $\begin{array}{l}\text { Percentage of area composed of low-intensity, moderate-intensity, and high- } \\
\text { intensity urbanization within each NHDPlus watershed containing the upstream } \\
\text { reach of each tailwater. Urban landcover based on the National Land Cover } \\
\text { Database } 2001 \text { (MRLC): http://www.mrlc.gov /datasets. Urban land cover } \\
\text { within each NHDPlus catchment was available from the National Fish Habitat } \\
\text { Action Plan Dataset (http://ecosystems.usgs.gov/fishhabitat/). }\end{array}$ \\
\hline $\begin{array}{l}\text { Population } \\
\text { Density }\end{array}$ & POPDENt & & $\begin{array}{l}\text { Estimated population density (individuals } / \mathrm{km}^{2} \text { ) within each NHDPlus } \\
\text { watershed containing the upstream reach of each tailwater. Based on US } \\
\text { Population Density } 2000 \text { data from the National Oceanic and Atmospheric } \\
\text { Administration: http://www.ngdc.noaa.gov/dmsp/download_sprawl.html. } \\
\text { Population density within each NHDPlus catchment was available from the } \\
\text { National Fish Habitat Action Plan Dataset } \\
\text { (http://ecosystems.usgs.gov/fishhabitat/). }\end{array}$ \\
\hline
\end{tabular}

Upstream L_DAMSt NumberNumber of dams occurring within NHDPlus watershed containing the upstream Reach Dams reach of each tailwater. Dam locations were based on the US Army Corps of Within Engineers National Dam Inventory

Watershed (http://www.usace.army.mil/Library/Maps/Pages/NationalInventoryofDams.as px). The number of dams per NHDPlus catchment was available from the National Fish Habitat Action Plan Dataset (http://ecosystems.usgs.gov/fishhabitat/).

N_DAMSt Number Number of dams occurring in the drainage network upstream of each NHDPlus watershed containing the upstream reach of each tailwater. Dam locations based on the US Army Corps of Engineers National Dam Inventory (http://www.usace.army.mil/Library/Maps/Pages/NationalInventoryofDams.as px). The number of dams occurring in the entire upstream network per each NHDPlus catchment was available from the National Fish Habitat Action Plan Dataset (http://ecosystems.usgs.gov/fishhabitat/).

Land LD_IDXi Number The Land Disturbance Index for each NHDPlus watershed containing the Disturbance Index upstream reach of each tailwater. Land disturbance indices were created for NHDPlus catchments through the National Fish Habitat Action Plan (http://ecosystems.usgs.gov/fishhabitat/). A cumulative disturbance index was created for approximately 2.23 million US river reaches (NHD flow lines) from landscape anthropogenic activities using urban areas, agricultural areas, roads, dams, mines, population density, and point-source pollution sites. Lower values indicate higher disturbance to aquatic habitats. 


\section{HS_NSD_Environmental: Attribute summary}

\begin{tabular}{|c|c|c|c|}
\hline Alias & Field Name & Unit & Description \\
\hline $\begin{array}{l}\text { NSD Environmental } \\
\text { Attributes ID }\end{array}$ & NSD_EnvID & ID & $\begin{array}{l}\text { Unique identification code assigned to each } \\
\text { HS_NSD_Environmental record. }\end{array}$ \\
\hline NSD Reach ID & ReachID & ID & $\begin{array}{l}\text { Unique stream-reach identifier used in the NSD assessment. The } \\
\text { last 4-digits of the ReachID are the Subregion HUC04 ID. }\end{array}$ \\
\hline Critical Habitats & CRITHABd & Number & $\begin{array}{l}\text { Number of critical habitats occurring within } 8,000 \mathrm{~m} \text { of the } \\
\text { potential dam location designated for potential hydropower } \\
\text { development. Critical habitats are lines or polygons designated } \\
\text { by the US Fish and Wildlife Service for federally listed species } \\
\text { under the ESA of } 1973 \text {. }\end{array}$ \\
\hline $\begin{array}{l}\text { Federally Listed Fish } \\
\text { Species }\end{array}$ & FISHESAd & Number & $\begin{array}{l}\text { Number of federally listed fish species potentially occurring } \\
\text { within the 8-HUC watershed containing each dam location. } \\
\text { Federally listed fish included species whose entire population or } \\
\text { subpopulation is listed as "endangered," "threatened," proposed } \\
\text { for listing, or a species of concern according to the ESA (1973). }\end{array}$ \\
\hline $\begin{array}{l}\text { Potadromous Fish } \\
\text { Species of Concern }\end{array}$ & FSHIUCNd & Number & $\begin{array}{l}\text { Number of fish species of concern potentially occurring within } \\
\text { the } 8 \text {-HUC watershed containing each dam location. Fish species } \\
\text { of concern were determined according to the IUCN Red List } \\
\text { Criteria. }\end{array}$ \\
\hline $\begin{array}{l}\text { Potadromous Fish } \\
\text { Species }\end{array}$ & FISHPOTd & Number & $\begin{array}{l}\text { Number of fish species documented as potadromous within the 8- } \\
\text { HUC watershed containing each dam location. Potadromous fish } \\
\text { are species migrating entirely in freshwater to complete their life } \\
\text { cycle requirements. }\end{array}$ \\
\hline $\begin{array}{l}\text { Conservation Lands } \\
\text { Near Potential Dam }\end{array}$ & PROTLNDd & $\mathrm{N} / \mathrm{A}$ & $\begin{array}{l}\text { An indication of whether protected conservation lands occur } \\
\text { within } 2,500 \mathrm{~m} \text { of potential dam locations designated for } \\
\text { potential hydropower development. " } 1 \text { " indicating "yes," and " } 0 \text { " } \\
\text { indicating "no." }\end{array}$ \\
\hline $\begin{array}{l}\text { Land Ownership } \\
\text { Index }\end{array}$ & LOWNIDXd & Number & $\begin{array}{l}\text { The Land Ownership Index is the sum of the number of different } \\
\text { governmental or nongovernmental entity lands that occur within } \\
2,500 \mathrm{~m} \text { of potential dam locations designated for potential } \\
\text { hydropower development. Entities include federal, state, } \\
\text { nongovernmental agency, local government, Native American, } \\
\text { regional agency, territorial, private conservation, joint ownership, } \\
\text { and unknown agency. Thus, the Land Ownership Index could } \\
\text { range from } 0 \text { (no protected land intersecting a stream reach) to } 10 \\
\text { (all entities have lands intersecting a stream reach). }\end{array}$ \\
\hline $\begin{array}{l}\text { Land Designation } \\
\text { Index }\end{array}$ & LNDDESGd & Number & $\begin{array}{l}\text { The Land Designation Index is the sum of the number of different } \\
\text { land designations that occur within } 2,500 \text { m of potential dam } \\
\text { locations designated for potential hydropower development. Land } \\
\text { designations include US National Park, US National } \\
\text { Forest/Grassland, National Trail, National Wildlife Refuge, } \\
\text { National Natural Landmark, National Landscape Conservation } \\
\text { System (Non Wilderness), National Landscape Conservation }\end{array}$ \\
\hline
\end{tabular}


National Parks Near NATPRKd

Potential Dam

Wild and Scenic WSRd N/A
Rivers Near Potential
Dam

$\begin{array}{lll}\text { 303d Listed } & \text { WQd } & \text { Number } \\ \text { Waterbodies Near } & & \\ \text { Potential Dam } & \end{array}$

Whitewater Boating

Runs Near Potential

Dam

Waterfalls Near
Potential Dam
Total Boat Ramps
Near Potential Dam

Fishing Access

Locations Near

Potential Dam

Estimated Surface

Water Use

Estimated

Groundwater Use

Urbanization Near

Potential Dam

AWRAFTd Number
System (Wilderness), Wild and Scenic Rivers, Marine Protected Area, Wilderness Area, Historic/Cultural Area, Military Land, Habitat or Species Management Area, Research Natural Area, Local Conservation, Private Conservation, and Conservation Program Land. Thus, the Land Designation Index could range from 0 (no protected land intersecting a stream reach) to 17 (all designation types intersect a stream reach).

N/A An indication of whether US National Parks occur within 2,500 $\mathrm{m}$ of potential dam locations designated for potential hydropower development. " 1 " indicating "yes," and " 0 " indicating "no."

An indication of whether US designated Wild and Scenic Rivers occur within $2500 \mathrm{~m}$ of potential dam locations designated for potential hydropower development. " 1 " indicating "yes," and " 0 " indicating "no."

Number of 303d listed waterbodies occurring within $500 \mathrm{~m}$ of potential dam locations designated for potential hydropower development. Under the Clean Water Act (1977), Section 303(d), states are required to specify designated uses for all waterways (e.g., public water supply, protection of fish and wildlife, recreation). In addition, each state must identify and adopt water quality criteria that support each designated use category and determine a list of streams that are not meeting their designated uses (303d List).

Number of whitewater boating runs (launch/take-out point combinations) occurring within $500 \mathrm{~m}$ of potential dam locations designated for potential hydropower development.

FALLSd Number

Number of waterfalls occurring within $800 \mathrm{~m}$ of potential dam locations designated for potential hydropower development.

BOATRMPd Number Number of developed and undeveloped boat ramps occurring within $500 \mathrm{~m}$ of potential dam locations designated for potential hydropower development.

FISHACCd Number Number of fishing access locations occurring within $500 \mathrm{~m}$ of potential dam locations designated for potential hydropower development.

Estimated surface water use $\left[(\mathrm{L} / \mathrm{d}) / \mathrm{km}^{2}\right]$ within the 8 -HUC watershed containing each dam location.

Estimated groundwater use $\left[(\mathrm{L} / \mathrm{d}) / \mathrm{km}^{2}\right]$ within the $8-\mathrm{HUC}$ watershed containing each dam location.

Percentage of area composed of low-intensity, moderateintensity, and high-intensity urbanization within each NHDPlus watershed containing each dam location. 
Estimated Population POPDEN Density

Dams Per Watershed L_DAMSd of Potential Dam

Dams Upstream of N_DAMSd Each Potential Dam

Land Disturbance LD_IDXd Index
Number Estimated population density (individuals $/ \mathrm{km}^{2}$ ) within each NHDPlus watershed containing each dam location.

Number Number of dams occurring within each NHDPlus watershed containing each dam location.

Number Number of dams occurring in the drainage network upstream of each NHDPlus watershed containing each dam location.

Number The Land Disturbance Index for each NHDPlus watershed containing each dam location. Land disturbance indices were created for NHDPlus catchments through the National Fish Habitat Action Plan (http://ecosystems.usgs.gov/fishhabitat/). A cumulative disturbance index was created for approximately 2.23 million US river reaches (NHD flow lines) from landscape anthropogenic activities using urban areas, agricultural areas, roads, dams, mines, population density, and point-source pollution sites. Lower values indicate higher disturbance to aquatic habitats. 


\section{HSDM1 CROSSWALK}

ORNL's HydroSource team has established a database crosswalk between HSDM1 data components and external frameworks. The HSDM1 crosswalk provides key linkages to major external database frameworks, which enables multiple capabilities for extending HSDM1 and providing added value for advancing hydropower research and supporting US hydropower sustainability. 


\section{Table 1. HSDM1 crosswalk}

\begin{tabular}{|c|c|c|c|}
\hline Data Component & Cross-Walk ID & External Source & Source Description \\
\hline HS_EF_Dam & FcDAMS_ID & \multirow{4}{*}{ SO_FERC } & \multirow{4}{*}{$\begin{array}{l}\text { The Federal Energy Regulatory Commission's (FERC) FERC DAMS Database (non-public), FERC } \\
\text { eLibrary, and FERC Form } 1 .\end{array}$} \\
\hline HS_EF_Plant & FcDecket & & \\
\hline HS_Mitigation & Fcuocket & & \\
\hline HS_PPA & ${ }^{*}$ FcForm1ID & & \\
\hline HS_EF_Plant & EIA_PtID & \multirow{2}{*}{ HSO_EIA } & \multirow{2}{*}{ Forms $860,906,920$, and 923 from the Energy Informaiton Administration (EIA). } \\
\hline HS_EF_Unit & EIA_UnID & & \\
\hline HS_EF_Dam & \multirow{2}{*}{ NID_ID1 } & \multirow{2}{*}{ SO_NID } & \multirow{2}{*}{$\begin{array}{l}\text { The National Inventory of Dams' (NID) congressionally authorized database documenting dams in } \\
\text { the United States and its territories. NID is maintained and published by the U.S. Army Corps of }\end{array}$} \\
\hline HS_NPD_Dam & & & \\
\hline HS_RefurbUpgrade & IIIR_PtID & \multirow{2}{*}{ - } & \multirow{2}{*}{$\begin{array}{l}\text { Industrial Info Resources (IIR) Database containing information on power, energy, and industrial } \\
\text { infrastructure markets. }\end{array}$} \\
\hline HS EF Turbine & IIR TrID & & \\
\hline HS_FleetIntel & EUCG_ID & SO_EUCG & $\begin{array}{l}\text { The Electric Utility Cost Group's (EUCG) Hydroelectric Productivity Committee (HPC) Database } \\
\text { containing hydropower performance and cost data. }\end{array}$ \\
\hline HS_FleetIntel & hydroAMP_ID & SO_hydroAMP & $\begin{array}{l}\text { Hydropower Asset Condition Assessments (hydroAMP) from the U.S. Bureau of Reclamation } \\
\text { (USBR) and U.S. Army Corps of Engineers (USACE). }\end{array}$ \\
\hline HS_mResFlowline & COMID & \multirow{3}{*}{ SO_NHDPlus } & \multirow{3}{*}{$\begin{array}{l}\text { The National Hydrography Dataset Plus (NHDPlus) is a geo-spatial, hydrologic framework dataset } \\
\text { built by the U.S. Environmental Protection Agency's (EPA) Office of Water, assisted by the U.S. } \\
\text { Geological Survey. }\end{array}$} \\
\hline HS_HUC_8 & HUC_8 & & \\
\hline HS_HUC_6 & HUC_6 & & \\
\hline HS_HUC_2 & HUC_2 & SO_WBD & $\begin{array}{l}\text { The Watershed Boundary Dataset (WBD) defines the areal extent of surface water drainage to a } \\
\text { point, accounting for all land and surface areas. }\end{array}$ \\
\hline *HS_HUC_8 & HUC_8 & SO_NRCS & Watershed boundary data generated by the Natural Resources Conservation Service (NRCS). \\
\hline HS_FleetIntel & *GADS_ID & SO_GADS & $\begin{array}{l}\text { Reliability information for total unit and major equipment groups from the North American } \\
\text { Electric Reliability Corporation's (NERC) Generating Availability Data System (GADS). }\end{array}$ \\
\hline
\end{tabular}

*HS_HUC_8 is listed twice to distinguish HUC-8 boundaries delineated from separate sources.

\begin{tabular}{|l|l|}
\hline \multicolumn{2}{|l|}{ Source Confidentiality } \\
\hline & Potential Future Linkage \\
\hline & Public and Nonpublic \\
\hline & Nonpublic \\
\hline & Public \\
\hline
\end{tabular}




\section{GLOSSARY}

\begin{tabular}{|c|c|}
\hline Term & Definition \\
\hline Attribute & $\begin{array}{l}\text { Refers to variables (e.g., height, weight, slope, material composition, age, } \\
\text { primary participant, and owner) associated with HSDM1 data components. }\end{array}$ \\
\hline Confidentiality & $\begin{array}{l}\text { Category classifying the general availability of each data component (i.e., } \\
\text { public, upon request, nonpublic, varies). "Public" denotes open access } \\
\text { HSDM1 data (i.e., free of cost or other barriers to acquisition). "Upon } \\
\text { request" denotes HSDM1 data available to anyone upon request. There are no } \\
\text { sharing restrictions for these data, but additional oversight is in place to help } \\
\text { ensure their appropriate usage. "Nonpublic" denotes HSDM1 data with } \\
\text { sharing restrictions that limit their availability to others (e.g., proprietary, } \\
\text { export-controlled, business sensitive, official use only). "Varies" denotes } \\
\text { HSDM1 data containing a mixture of public and nonpublic information. }\end{array}$ \\
\hline Database indices & $\begin{array}{l}\text { Index fields that compose data components (i.e., primary key, foreign key, } \\
\text { external crosswalk, potential future key). }\end{array}$ \\
\hline Data components & $\begin{array}{l}\text { Primary building blocks that make up the HSDM1 schema. They are } \\
\text { essentially different types of data objects that store (1) related sets of } \\
\text { attributes and (2) database indices (i.e., primary key, foreign key, external } \\
\text { crosswalk key potential future key) that provide connectivity among and } \\
\text { beyond data components within HSDM1. }\end{array}$ \\
\hline External crosswalk key & The data component index field is an external crosswalk key. \\
\hline Foreign key & The data component index field is a foreign key. \\
\hline Geospatial point layer & $\begin{array}{l}\text { Data component type representing discrete geospatially referenced locations } \\
\text { of zero-dimensional common features (e.g., addresses, place names). }\end{array}$ \\
\hline Geospatial polygon layer & $\begin{array}{l}\text { Data component type representing discrete geospatially referenced locations } \\
\text { of common area features (e.g., hydropower project boundaries, waterbodies, } \\
\text { land ownership, land cover). }\end{array}$ \\
\hline Geospatial polyline layer & $\begin{array}{l}\text { Data component type representing discrete geospatially referenced locations } \\
\text { of common linear features (e.g., flow lines, transmission lines, roads). }\end{array}$ \\
\hline HSDM1 crosswalk & $\begin{array}{l}\text { Provides key linkages to major external database frameworks, enabling } \\
\text { multiple capabilities for extending HSDM1 and providing added value for } \\
\text { advancing hydropower research and supporting US hydropower } \\
\text { sustainability. }\end{array}$ \\
\hline
\end{tabular}


Nonspatial table

Potential future key

Primary key

Properties

Relational table

Research theme

Time-series
Data component type representing tabular data that contain a related set of attributes. Nonspatial tables do not explicitly represent geospatially referenced locations of a common feature type, contain time-series data, or function as a relational table within HSDM1.

The data component index field is a potential future key.

The data component index field is a primary key.

Description of attributes and database indexes within HSDM1 (i.e., field, alias, unit of measurement, description).

Data component type that stores and indexes unique index fields from two separate data components to enable a many-to-many relationship.

Primary subject matter pertaining to a common overarching goal(s) of WPTO-funded ORNL research projects.

Data component type representing a set of attributes containing time-series information. Time-series data components within HSDM1 are all nonspatial because they do not explicitly represent geospatially referenced locations of a common feature type. 